\title{
Hearing shapes of drums - mathematical and physical aspects of isospectrality
}

Olivier Giraud

Laboratoire de Physique Théorique, UMR 5152 du CNRS, Université Paul Sabatier, 31062 Toulouse Cedex 4, France*

Koen Thas

Ghent University,Department of Pure Mathematics and Computer Algebra, Krijgslaan 281, S22, B-9000 Ghent,Belgium ${ }^{\dagger}$

(Dated: August 6, 2007)

\begin{abstract}
In a celebrated paper "Can one hear the shape of a drum?" (Amer. Math. Monthly 73 (1966), 1-23) M. Kac asked his famous question on the consequences of isospectrality, which was eventually answered negatively by construction of non-congruent planar isospectral pairs.
\end{abstract}

*Electronic address: giraud@irsamc.ups-tlse.fr

${ }^{\dagger}$ Electronic address: kthas@cage. UGent. be 


\section{Contents}

Relation between Shapes and Sounds of Drums, According to M. Kac 6

$\begin{array}{ll}\text { A. One-dimensional systems } & 6\end{array}$

$\begin{array}{ll}\text { B. Vibrating plates experiments (Chladni) } & 7\end{array}$

$\begin{array}{ll}\text { C. Physical problems } & 7\end{array}$

D. Quantum chaos 8

E. Mathematical problem $\quad 11$

I. A pedestrian proof of isospectrality 12

$\begin{array}{ll}\text { A. Paper-folding proof } & 12\end{array}$

B. Transplantation proof 16

II. Further Examples in Higher Dimensions 22

A. Lattices and flat tori $\quad 22$

B. Construction of examples 24

C. The eigenvalue spectrum as moduli for flat tori 25

$\begin{array}{ll}\text { III. Sunada Theory } & 27\end{array}$

$\begin{array}{lr}\text { A. Permutations } & 27\end{array}$

$\begin{array}{ll}\text { B. Commutator notions } & 27\end{array}$

C. $p$-Groups and extra-special groups 28

D. Finite simple groups 29

$\begin{array}{ll}\text { E. Sunada Theory } & 30\end{array}$

F. Sunada's theorem and a trace formula

G. Property (*): examples 33

IV. Livsic Cohomology 36

A. Group cohomology 36

Group modules $\quad 36$

$\begin{array}{ll}\text { The } n \text {-th cohomology group } & 37\end{array}$

B. Livsic's cohomological equation 39

$\begin{array}{ll}\text { V. Properties of isospectral billiards } & 40\end{array}$ 
$\begin{array}{lr}\text { A. Weyl expansion } & 41\end{array}$

$\begin{array}{ll}\text { B. Periodic orbits } & 43\end{array}$

$\begin{array}{lr}\text { 1. Green function } & 43\end{array}$

2. Semiclassical Green function $\quad 45$

$\begin{array}{lr}\text { 3. Density of states } & 47\end{array}$

$\begin{array}{lr}\text { C. Diffractive orbits } & 50\end{array}$

$\begin{array}{lr}\text { D. Green function } & 52\end{array}$

E. Scattering poles of the exterior Neumann problem 53

$\begin{array}{ll}\text { 1. Fredholm theory } & 53\end{array}$

$\begin{array}{lr}\text { F. Eigenfunctions } & 56\end{array}$

$\begin{array}{ll}\text { 1. Triangular states } & 56\end{array}$

$\begin{array}{ll}\text { 2. Mode-matching method } & 57\end{array}$

$\begin{array}{ll}\text { G. Eigenvalue statistics } & 61\end{array}$

$\begin{array}{lr}\text { H. Nodal domains } & 64\end{array}$

VI. Experimental and numerical investigations 65

A. Numerical investigations $\quad 66$

1. Numerical computations of the spectrum and eigenfunctions by a mode-matching method 66

2. Numerical computations by expansion of eigenfunctions around the corners, with domain-decomposition method 66

$\begin{array}{lr}\text { B. Experimental realizations } & 67\end{array}$

1. Electromagnetic waves in metallic cavities $\quad 67$

2. Transverse vibrations in vacuum for liquid crystal smectic films 69

$\begin{array}{ll}\text { VII. Transplantation } & 71\end{array}$

$\begin{array}{ll}\text { A. Graphs and billiards by tiling } & 71\end{array}$

$\begin{array}{ll}\text { B. The example of Gordon et al. } & 73\end{array}$

$\begin{array}{lr}\text { C. The other known examples } & 75\end{array}$

D. Euclidean TI-domains and their involution graphs $\quad 75$

$\begin{array}{ll}\text { E. Finite projective geometry } & 77\end{array}$

$\begin{array}{ll}\text { F. Axiomatic projective spaces } & 78\end{array}$

$\begin{array}{lr}\text { G. Automorphism groups } & 79\end{array}$ 
H. Involutions in finite projective space $\quad 80$

I. Transplantation matrices, projective spaces and isospectral data 81

J. Generalized isospectral data $\quad 86$

$\begin{array}{ll}\text { K. The operator group } & 86\end{array}$

$\begin{array}{ll}\text { VIII. Related questions } & 88\end{array}$

$\begin{array}{lr}\text { A. Boundary conditions } & 88\end{array}$

B. Isospectrality versus iso-length spectrality 90

Okada and Shudo's result on iso-length spectrality 90

Penrose-Lifshits mushrooms $\quad 92$

C. Spectral problems for Lie geometries $\quad 95$

$\begin{array}{ll}\text { D. Generalized polygons } & 96\end{array}$

$\begin{array}{lr}\text { Definition and properties } & 97\end{array}$

$\begin{array}{lr}\text { Duality principle } & 99\end{array}$

$\begin{array}{lr}\text { Automorphisms and isomorphisms } & 99\end{array}$

$\begin{array}{lr}\text { Collinearity matrices } & 99\end{array}$

$\begin{array}{lr}\text { E. Point spectra and order } & 100\end{array}$

$\begin{array}{lr}\text { Case } n=3 & 100\end{array}$

$\begin{array}{lr}\text { Case } n=4 & 101\end{array}$

$\begin{array}{lr}\text { Case } n=6 & 101\end{array}$

$\begin{array}{lr}\text { Case } n=8 & 103\end{array}$

$\begin{array}{lr}\text { F. Concluding remarks } & 103\end{array}$

$\begin{array}{lr}\text { G. Homophonic pairs } & 106\end{array}$

$\begin{array}{lr}\text { IX. Open Problems } & 106\end{array}$

$\begin{array}{lr}\text { Acknowledgments } & 108\end{array}$

$\begin{array}{lr}\text { A. The finite simple groups } & 109\end{array}$

$\begin{array}{ll}\text { B. Gallery of examples } & 111\end{array}$

$\begin{array}{lr}\text { 1. Some modes } & 111\end{array}$

2. The 17 families of isospectral pairs and their mathematical properties 111 


\section{Relation between Shapes and Sounds of Drums, According to M. Kac}

In many fields of physics, such as quantum mechanics or electromagnetics, the stationary Schrödinger equation, or Helmholtz equation

$$
(\Delta+\mathbf{E}) \Psi=0
$$

plays a central role. For two-dimensional domains, in the framework of quantum chaos, semiclassical trace formulas (see e.g. (Gutzwiller, 1990)) provide a connection between the density of energy levels and classical features of the domains such as area, perimeter, and classical trajectories of a particle in the domain. Two domains sharing the same spectrum must therefore share common classical features. But do these domains have to be identical? In a celebrated paper "Can one hear the shape of a drum?" (Amer. Math. Monthly 73 (1966), 1-23) (Kač, 1966), M. Kac formulated his famous question

"Can one hear the shape of a drum?".

Formally, answering "no" to this question amounts to finding planar isospectral pairs - non-isometric planar simply connected domains for which the sets $\left\{E_{n} \| n \in \mathbb{N}\right\}$ of solutions of (1) with $\Psi_{\mid \text {Boundary }}=0$ are identical. Any example of such a pair of non-congruent planar isospectral domains yields a counter example to Kac's question.

Since the appearance of (Kač, 1966), more than 500 papers have been written on the subject.

TO PUT SOMEWHERE IN THE INTRODUCTION:

\section{A. One-dimensional systems}

The frequency spectrum uniquely determines the length of the system. Mathematically, solutions of the Laplace equation on an interval of length $L$ are $\operatorname{simply}$ given by $\sin (n \pi x / L)$. ETC.... 

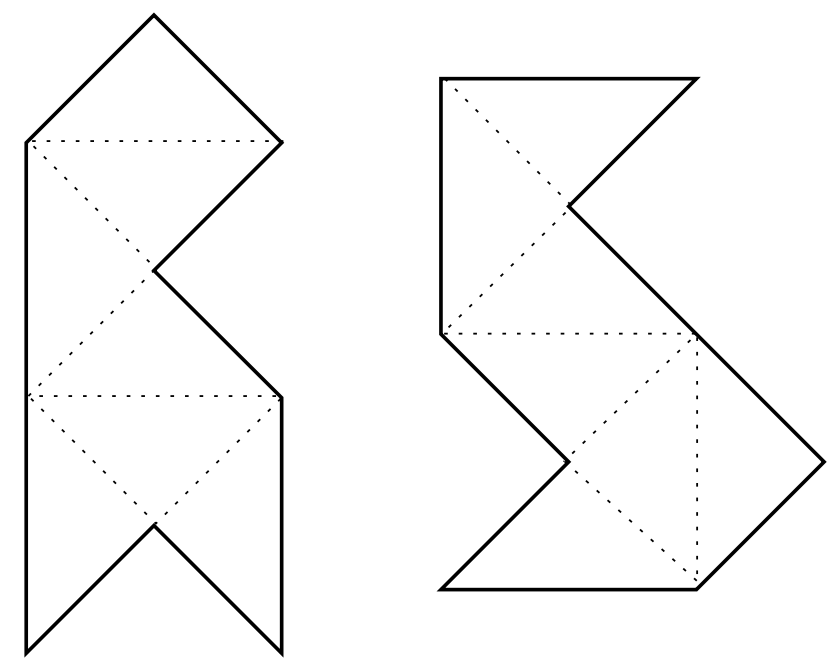

Figure 1 The paradigmatic pair of isospectral billiards with seven half-square shaped base tiles.

\section{B. Vibrating plates experiments (Chladni)}

\section{Physical problems}

- Scalar problem: vibrating membranes (Helmholtz equation for acoustical modes with $E=k^{2}$ and $k=2 \pi \nu / c$, where $\nu$ is the frequency and $c$ the velocity of sound), quantum mechanics (time-independent Schrödinger equation)

- Electromagnetic problem: for a cavity with perfectly reflecting walls, an electromagnetic wave verifies Maxwell equations... with boundary conditions $E_{\perp}=\ldots$. Here $k=2 \pi \nu / c$, with $c$ the speed of light;

- billiards.

Billiards are two-dimensional closed compact domains of the Euclidean plane $\mathbb{R}^{2}$. We will be mainly concerned here with systems modeling the behaviour of a particle in a box, whose dimensions are such that it can be assumed that it can be approximated by a twodimensional enclosure. The behaviour of the particle can be described, in the framework of quantum mechanics, by a wavefunction $\psi$, which is a function of the position of the particle, and which characterizes the amplitude of probability $\psi(x)$ that the particle be located at position $x$. If the system is described by the Hamiltonian $H$, the wavefunction 
satisfies the stationary Schrödinger equation

$$
(-H+E) \psi=0 .
$$

For two-dimensional billiards, defined by a closed contour $\partial B$ in the plane, the Hamiltonian describes the free motion inside the billiard and hard-wall boundary conditions; it reads $H=p^{2} / 2 m$ inside the boundary enclosure $\partial B, \infty$ outside. The study of billiards consists of looking for solutions of equation (2) for the free motion of a particle of mass $m$ inside the billiard, imposing boundary conditions on $\partial B$. Using units such that $\hbar=1$ and $m=1 / 2$, the stationary Schrödinger equation then reads

$$
(\Delta+E) \psi=0 \text {. }
$$

We will consider

- Dirichlet boundary conditions $\psi_{\mid \partial B}=0$;

- Neumann boundary conditions $\nabla \psi_{\mid \partial B}=0$.

Solving equation (3) gives eigenfunctions $\psi_{n}$ and eigenvalues $E_{n}$; if boundary conditions are imposed there is as infinite but countable number of real positive eigenvalues: we note $0<E_{1} \leq E_{2} \leq E_{3} \ldots$. Apart from some very special cases (e.g. the rectangular billiard), the analytical calculation of eigenvalues or eigenfunctions is practically impossible. However their statistical behaviour can be characterized, as we will see later.

\section{Quantum chaos}

In his work "Essai philosophique sur les probabilités", the French philosopher and mathematician Pierre Simon de Laplace writes in 1776 that "If we could imagine an intelligence that, for a given time, would capture all relations between beings in this universe, it could determine for any given time in the past or in the future the respective position, movements and, more generally, characteristics of all these beings." Laplacian determinism prevailed up to the end of the 19th century, when the mathematician Jacques Hadamard 
stressed the existence of systems for which time evolution depended strongly on initial conditions, which forbids any long-term prediction. In his book "Science et Méthode" (1908), Henri Poincaré states the fact that "A cause so small that it escapes our attention may determine a considerable effect that we are to see, and then we say that this effect is due to chance." Classical systems are thus sensitive to initial conditions, which make their evolution impredictable; very quickly chaos appears. This has led to the birth of new fields such as the study of stochastic systems, whose aim is at finding, through a statistical approach of phenomena, laws that govern chaos. Poincaré distinguishes between integrable systems, whose equations of motion can be made equivalent, by an appropriate change in variables, to a set of independent systems with one degree of freedom. The equations of motion for systems with one degree of freedom can be solved analytically, and therefore integrable systems are characterized by the fact that their equations of motion can be solved exactly. In integrable systems, trajectories occur in families. On the other hand, systems whose equations of motion are nonlinear do not admit generically analytical solutions: they are called non-integrable. In general, trajectories do not occur in families. They are isolated, and chaotic, which means that two trajectories initially very close can quickly diverge. For instance, the hydrogen atom, whose classical description can be done in terms of a two-body problem, is integrable, whereas the helium atom is more complex and non-integrable. This distinction establishes two classes in the world of classical mechanics. One is the class of integrable systems, whose equations of motion are exactly solvable, and which are governed by predictability. The other contains all non-integrable systems, among which chaos seems to be a characteristic.

The intrinsic unpredictability conferred to non-integrable systems by this chaotic behaviour has met the quantum revolution at the beginning of the twentieth century. Heisenberg's uncertainty principle makes any microscopic determinism impossible: according to this principle, it is impossible to know with arbitrary accuracy both the momentum and the position of a particle. However, the very notion of a trajectory looses its meaning in quantum mechanics. The evolution of a system is governed by a Schrödinger equation, which is a linear equation describing equally hydrogen and helium atoms. A system is described by the Hamiltonian and its eigenvalues and eigenfunctions which contain all information about its evolution. Since wavefunctions represent probability density amplitudes, this description is probabilistic by nature. But the statistical description that allowed 
to circumvent the lack of knowledge on a system because of its chaotic behaviour does not exist any more in quantum mechanics. Thus, quantum mechanics seems to ignore the distinction between chaotic and integrable systems.

However, a detailed study of experimental data coming from neutron scattering on heavy atomic nuclei revealed that such processes, which are chaotic, posess a local density of energy levels which coincides with the distribution of eigenvalues of random matrices (that is, matrices with independently distributed random coefficients). On the other hand, integrable systems, like for instance an electron in a rectangular box, displayed a local density of energy levels distributed like independent random variables. According to their classical behaviour, systems thus appeared to be characterized by very different energy level distributions. In particular, for independent random variables the probability for two levels to be very close is huge, whereas eigenvalues of random matrices have a characteristic behaviour called "level repulsion"; that is, the probability for two energy levels (or eigenvalues) to be close to each other goes to zero when the distance between nearest levels vanishes. At the beginning of the eighties, it became clear that there was indeed a quantum evidence of classical characteristics (integrability or chaoticity) of a system, and that the classical behaviour governed e.g. the properties of the local density of energy levels.

Numerical and experimental study of many various systems has confirmed the existence of two classes for the distribution of energy levels, corresponding to the two distinct classical behaviours of integrable or chaotic systems: all integrable systems on the one hand, all chaotic systems on the other hand, have a similar quantum behaviour. One can naturally wonder about the reasons of this correspondence, which is a manifestation of the deep connection between the classical and the quantum world. It is well known that classical mechanics can be seen as a limit of quantum mechanics when Planck's constant, seen as a parameter, goes to zero. It is therefore natural that, for small enough values of this parameter, classical characteristics of quantum systems begin to emerge. If one considers an electron in a box, one can construct a certain linear combination of wave functions that describes its probability density distribution at each point of the box. At the classical limit, this probability distribution gets localized (that is, takes higher values) on classically authorized trajectories. The quantum systems thus somehow "knows" about classical trajectories of the underlying classical system; it is therefore quite natural that the statistical 
behaviour of quantum energy levels be, at least partly, related to the classical features of the system. It should be briefly mentioned that there is a great variety of systems beyond integrable and chaotic ones. In particular, non-integrable systems can display various degrees of chaos; some are not even chaotic. This quantum-classical correlation can be understood in a less handwaving way, in the framework of quantum chaos, by a tool called trace formula, which will be the subject of section V.B.2.

As the study of isospectral billiards wasa historically mainly concerned with polygonal billiards, it should be mentioned that there exists in particular a class of billiards called pseudo-integrable billiards. These are both non-integrable and non-chaotic billiards, and their classical characteristics intermediate between those of integrable and those of chaotic billiards. For instance, classical trajectories appear within families of parallel trajectories of same length, but nevertheless the equations of motion are not exactly solvable because of the presence of diffraction corners. The quantum study of such diffractive systems has revealed that for many of them the energy level nearest-neighbour distribution also displayed properties intermediate between those of integrable and those of chaotic billiards. In particular they display level repulsion, like chaotic billiards or eigenvalues of random matrices; but the nearest-neighbour distribution has only an exponential decrease, like for integrable systems or randomly distributed variables. These distributions are called "intermediate statistics".

\section{E. Mathematical problem}

harmonic functions (solutions of Laplace equation).

- Dirichlet problem

- Neumann problem (also called in old papers hydrodynamic problem)

- mixed boundary conditions: Zaremba (Zaremba, 1927) proposed in 1927 the generalized problem of finding, for a given $L^{2}$ vector field $V$, a harmonic $L^{2}$ function $u$ such that $\forall h$ harmonic function $\int(V-\nabla \cdot u) \cdot|\nabla h|=0$. He showed that if $\operatorname{div} V=0$ in the whole domain this problem is equivalent to Neumann problem. 


\section{A PEDESTRIAN PROOF OF ISOSPECTRALITY}

The first examples of isospectral billiards in the Euclidean plane were constructed by using powerful mathematical tools. We will however postpone this historical construction to section III.3. This sections aims at presenting the main ideas that are involved in isosectrality, so that the reader can acquire some intuition on it. More rigorous mathematical grounds will be provided in the next section.

\section{A. Paper-folding proof}

We shall start with a simple construction method that was proposed in (Chapman, 1995). It is based on the so-called "paper-folding" method. To illustrate it we will follow (Thain, 2004), where the method is illustrated for a simple example. Consider the two billiards

\begin{tabular}{|l:l:l|l|}
\hline 1 & 2 & 3 & 4 \\
\cline { 2 - 3 } & 5 & 6 & 7 \\
\hline
\end{tabular}

\begin{tabular}{|l|l|l|l|l|}
\hline 1 & 2 & 3 & 4 & \\
\hline & 5 & 6 & 7 \\
\hline
\end{tabular}

Figure 2 The pair $7_{3}$ (see appendix B) of isospectral billiards with a rectangular base shape.

in Fig. 2. Each billiard is made of seven identical rectangular building blocks. The solid lines are hard wall boundaries, the dotted lines are just an eyeguide marking the building blocks. Let $\phi$ be an eigenfunction of the left billiard with energy $E$. The goal is to construct an eigenfunction of the right billiard with the same energy, that is a function which

- verifies Eq. (3);

- vanishes on the boundary of the billiard;

- has a continuous normal derivative inside the billiard.

The idea is to define a function $\psi$ on the right billiard as a superposition of translations of the function $\phi$. Since Helmholtz equation (3) is linear, any linear combination of translations of $\psi$ will be a solution of Helmholtz equation with the same eigenvalue $E$ in the interior of each building block of the second billiard. The problem reduces to find a linear combination that vanishes on the boundary and has the correct continuity properties 
inside the billiard. The following method allows to obtain all these properties simultaneously. Take three copies of the left billiard of Fig. 2. Each copy is then folded in a different
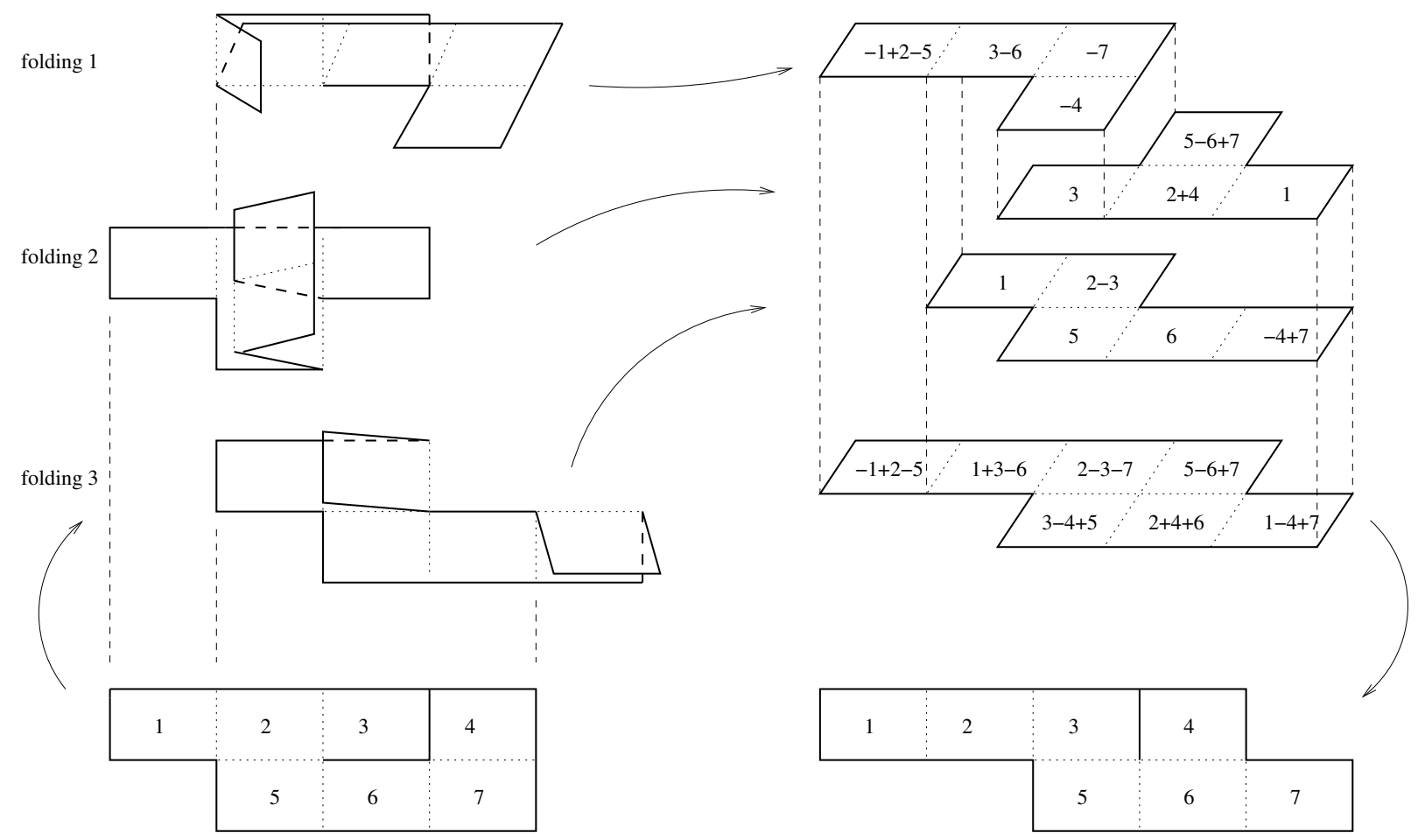

Figure 3 Pictorial representation of the paper-folding method

way, as shown in Fig. 3 (left column). Then the three folded billiards are stacked on top of each other as indicated in the right column of Fig. 3; note that the first shape (folding 1) has been translated on the left before being stacked, and that the second shape (folding 2) has been rotated by $\pi$. Once superposed, these three billiard yield the shape of the right billiard of Fig. 2.

Now we make a correspondence between stacking two sheets of paper and adding the functions defined on these sheets; moreover stacking the reverse of a sheet corresponds to adding the opposite of the function. For instance in folding 3, a minus sign is associated in the right column to tiles 3 and 4 , which are folded back, and a plus sign to the other tiles which are not. The function $\psi$ is defined by this "folding and stacking" procedure. For instance is is defined in the tile numbered 1 in Fig. 2 by

$$
\left.\psi\right|_{\mathrm{t} i l e 1}=-\left.\phi\right|_{\mathrm{t} i l e 1}+\left.\phi\right|_{\mathrm{tile2}}-\left.\phi\right|_{\mathrm{t} i l e 5} .
$$


The procedure above ensures that $\psi$ vanishes on the boundary and has a continuous derivative across the tile boundaries. Consider for instance the leftmost vertical boundary of the right billiard. We have $\left.\phi\right|_{\text {tiles }}=0$ on this boundary (since it is at the boundary of the left billiard), and $\left.\phi\right|_{\text {tile } 1}=\left.\phi\right|_{\text {tile2 }}$ on this boundary since tiles 1 and 2 are glued together. Thus, $\psi$ given by Eq. (4) indeed vanishes on the leftmost vertical boundary of the right billiard.

With the paper-folding method, it is clear that what matters is the way the building blocks (the elementary rectangles in our example) are glued to each other, irrespective of their shape. Suppose we denote by 1, 2, 3 respectively the left, right and bottom edge of tile 4 in the left billiard of Fig. 2. To obtain the whole billiard one unfolds tile 4 with respect to its side number 3 , getting tile 7 . Then tile 7 is unfolded with respect to its side number 2 , yielding tile 6 , and so on. The unfolding rules can be summed up in a graph specifying the way we unfold the building block. The vertices of the graph represent the building blocks, and the edges of the graph are "coloured" according to the unfolding rule, i.e. according to which of its sides the building block is unfolded. The graphs can also be encoded in permutations $a^{(\mu)}, b^{(\mu)}, 1 \leq \nu \leq 3$. For instance for the first graph we have $a^{(1)}=(23)(56), a^{(2)}=(12)(67), a^{(3)}=(25)(47)$. It will turn out later to be useful to write these permutations as permutation matrices $M^{(\mu)}, N^{(\mu)}, 1 \leq \nu \leq 3$, with entries 0 and 1 . In fact, only three sides of the rectangle are involved in the unfolding. So we can start with any triangular-shaped building block, and unfold it with respect to its sides in the same way as the billiards in Fig. 2 are obtained from the rectangular building block. This leads to billiard pairs whose isospectrality is granted by the paper-folding proof given above. For example, starting from the triangle in Fig. 4) left and following these unfolding rules, we get the pair of isospectral billiards shown in Fig. 4) right. Clearly the paper-folding proof applies in exactly the same way as is does for the billiards of Fig. 2. Taking a building block in the form of half-square, we recover the celebrated example of Fig. 1.

The building block is in fact not required to be a triangle or a rectangle. More generally, any building block posessing three edges around which to unfold it leads to a different pair of isospectral billiards. Another interesting example is obtained by taking an heptagon and unfolding it with respect to three of its sides following the unfolding rules (see Fig. 5). This yields the first example of Gordon, Webb and Wolpert (Gordon et al., 1992). 

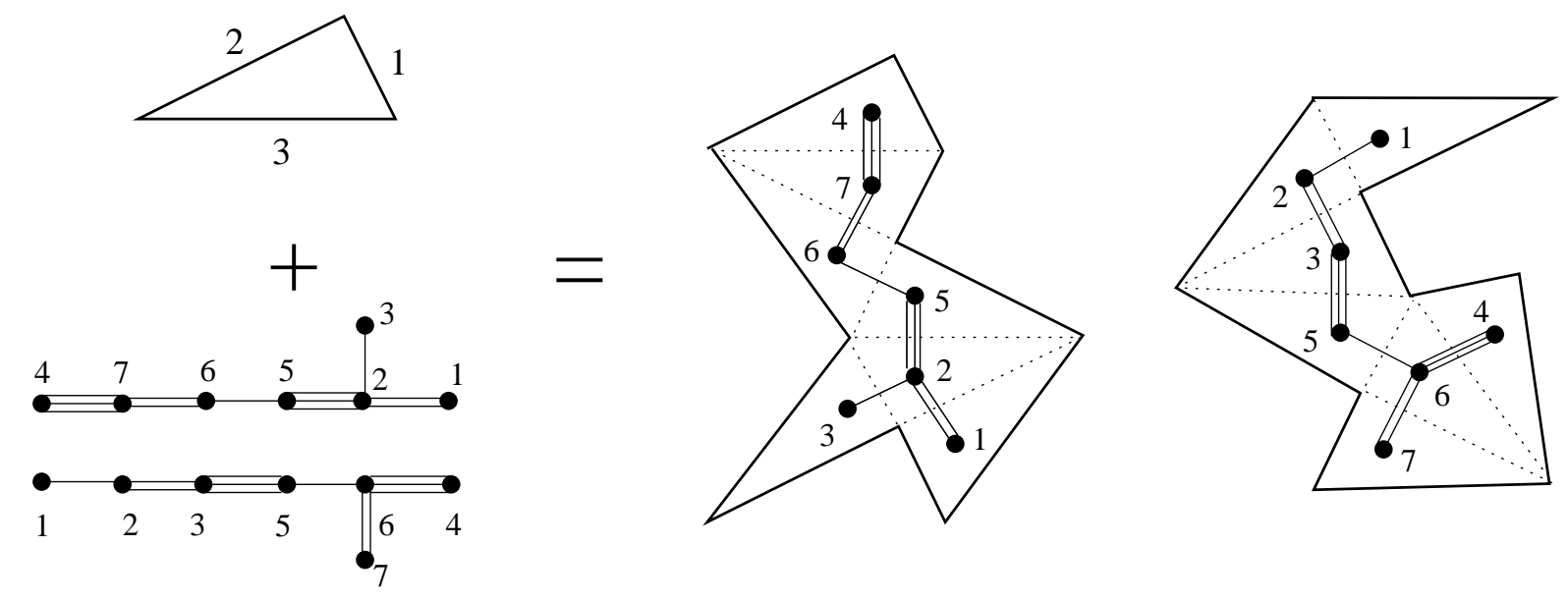

Figure 4 The graphs corresponding to a pair of isospectral billiards: if we label the sides of the triangle by $\mu=1,2,3$, the unfolding rule by symmetry with respect to side $\mu$ can be represented by edges made of $\mu$ braids in the graph. From a given pair of graphs, one can construct infinitely many pairs of isospectral billiards by applying the unfolding rules to any shape.

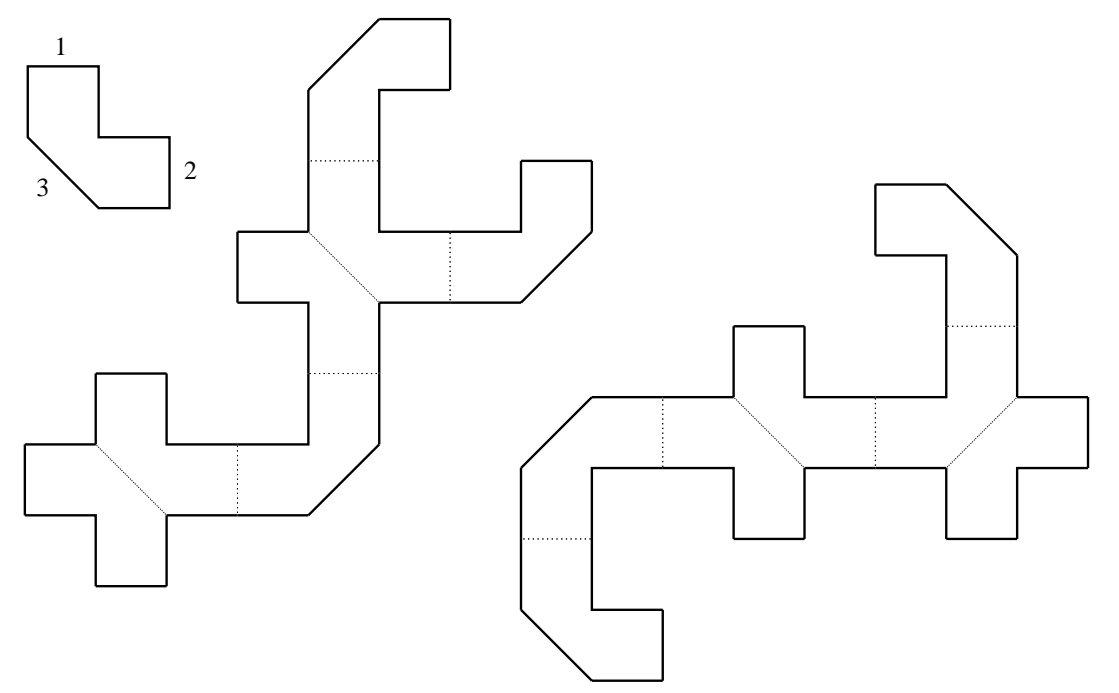

Figure 5 Isospectral billiards produced by Gordon, Webb and Wolpert (Gordon et al., 1992). The top left figure is the seven-edged building block.

In (Chapman, 1995), more involved examples are produced, following the same procedure. Starting from the building block of Fig. 6 left, one obtains an example of a pair of chaotic billiards. The building block of Fig. 6 right yields a very simple disconnected pair where each billiard consists of a disjoint rectangle and triangle. In this case isospectrality can be checked directly by calculating the eigenvalues, since the eigenvalue problem can be solved exactly for triangles and half-squares. 
In (Sleeman and Hua, 2000), the authors consider a building block with piecewise fractal boundary: starting from a $(\pi / 2, \pi / 3, \pi / 6)$ base triangle they cut each side into 3 pieces and remove the three triangular corners. Along the freshly made cuts a Koch curve is constructed, while the ancient sides still allow the Chapman unfolding. This yields a pair of isospectral billiards with fractal boundary of dimension $\log 4 / \log 3$.
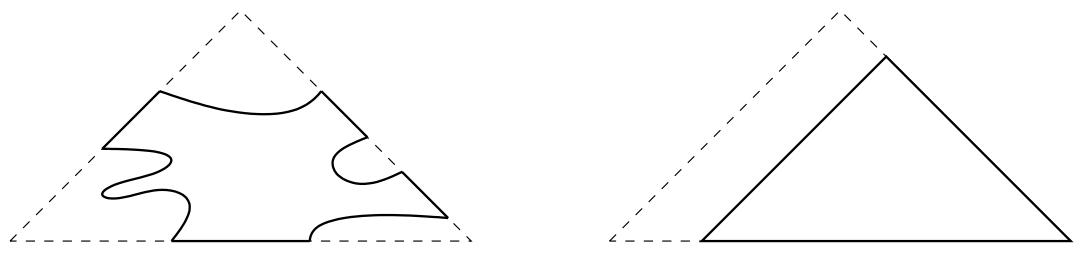

Figure 6 Examples of building blocks yielding isospectral pairs.

\section{B. Transplantation proof}

The paper-folding proof can be made more formal be means of the so-called "transplantation" method. The transplantation method was introduced by Bérard in (Bérard, 1989). It will be presented in more detail in section VII.

Let $\phi$ be an eigenstate of the first billiard. Any point in the billiard can be specified by its coordinates $\mathbf{q}=(x, y)$ inside a building block, and a number $i$ arbitrarily associated to the building block (for example $1 \leq i \leq 7$ in our example of Fig. 2). Thus $\phi$ is a function of the variable $(\mathbf{a}, i)$. According to the paper-folding proof, a building block $i$ of the second billiard is constructed from superposition of three building blocks $j$ obtained by folding the first billiard. We can code the result of the folding-and-stacking procedure in a so-called 
transplantation matrix $T$, as

$$
T=\left(\begin{array}{ccccccc}
-1 & 1 & 0 & 0 & 1 & 0 & 0 \\
1 & 0 & 1 & 0 & 0 & -1 & 0 \\
0 & 1 & -1 & 0 & 0 & 0 & -1 \\
0 & 0 & 0 & 0 & 1 & -1 & 1 \\
0 & 0 & 1 & -1 & 1 & 0 & 0 \\
0 & 1 & 0 & 1 & 0 & 1 & 0 \\
1 & 0 & 0 & -1 & 0 & 0 & 1
\end{array}\right)
$$

Isospectrality arises from the fact that one can construct an eigenstate $\psi$ of the second billiard, defined by

$$
\psi(a, i)=\mathcal{N} \sum_{j} T_{i j} \phi(a, j)
$$

where $\mathcal{N}$ is some normalization factor. That is, one can "transplant" the eigenfunction of the first billiard to the second one. The proof of isospectrality reduces to checking that $\psi$ given by (5)-(6) vanishes on the boundary and has a continuous derivative inside the billiard.

This is most simply illustrated on the following example, based on the presentation of (Thain, 2004). We consider the pair of Fig. 2 with rectangular base tile. The base shape could be replaced by any arbitrary shape provided only three sides are considered for applying unfolding rules leading to the construction of the pairs. Here we take a base rectangle with sides of length $a$ and $b$. The first step is to transform the problem into an equivalent one on translation surfaces. Translation surfaces (Gutkin and Judge, 2000), also called planar structures, are manifolds of zero curvature (i.e. such that in the vicinity of each point there exists a homeomorphism from this vicinity to $\mathbb{R}^{2}$ defining a local coordinate system), with a finite number of singular points (see (Vorobets, 1996) for a more rigorous mathematical definition). A construction by Zemlyakov and Katok (Zemlyakov and Katok, 1976) allows to construct a planar structure on rational polygonal billiards, that is polygonal billiards whose angles at the vertices are of the form $\alpha_{i}=\pi m_{i} / n_{i}$. This planar structure is obtained by "unfolding" the polygon, that is by gluing to the initial polygon its images obtained by mirror reflexion with respect to each of its sides, and repeating this process on the images. For polygons with angles $\alpha_{i}=\pi m_{i} / n_{i}$, this process 
terminates and $2 n$ copies of the initial polygon are required, where $n$ is the gcd of the $n_{i}$. Identifying parallel sides, one gets a planar structure of genus in general greater than 1 . This structure has singular points corresponding to vertices of the initial polygon where the angle $\alpha_{i}=\pi m_{i} / n_{i}$ is such that $m_{i} \neq 1$. The genus of the translation surface thus obtained is given by (Berry and Richens, 1981)

$$
g=1+\frac{n}{2} \sum_{i} \frac{m_{i}-1}{n_{i}} .
$$

The billiards of Fig. 2 possess one $2 \pi$-angle, two $3 \pi / 2$-angles and eight $\pi / 2$-angles each. The translation surfaces associated to these billiards are obtained by gluing together $2 n=$ 4 copies of the billiards, yielding planar surfaces of genus 4 . They are shown in Fig. 7. Opposite sides are identified (e.g. in the first surface, the left edge of tile 1 is identified with the right edge of tile 5). The symbols $\circ$ and $\bullet$ represent a $6 \pi$-angle, while the $\times$ and $*$ symbols denote a $8 \pi$ angle. Taking into account the identification of opposite sides, it means that one has to turn around $*$ by an angle $8 \pi$ before coming back to the initial point. An example of a staight line drawn on the first surface is shown on Fig. 7.

Each translation surface is tiled by 7 rectangles. Again, any point on the surface can be specified by its coordinates $\mathbf{q}=(x, y) \in[0, a] \times[0, b]$ inside the rectangle, together with a tile number $i$. Each tile on the translation surface has 6 neighbours, attached respectively at its left, upper left, upper right, right, lower right and lower left edge, and numbered from 1 to 6 . For instance tile 1 is surrounded by: tile 5 on its left, tile 6 on its right, tile 3 on its upper left edge, tile 1 itself on its upper right edge (because of the identification of opposite sides), tile 3 on its lower left edge and tile 1 on its lower right edge. The way the tiles are glued together can be specified by permutation matrices $A^{(\nu)}, 1 \leq \nu \leq 6$, such that $A_{i j}^{(\nu)}=1$ if and only if the edge number $\nu$ of $i$ glues $i$ and $j$ together. For instance for 

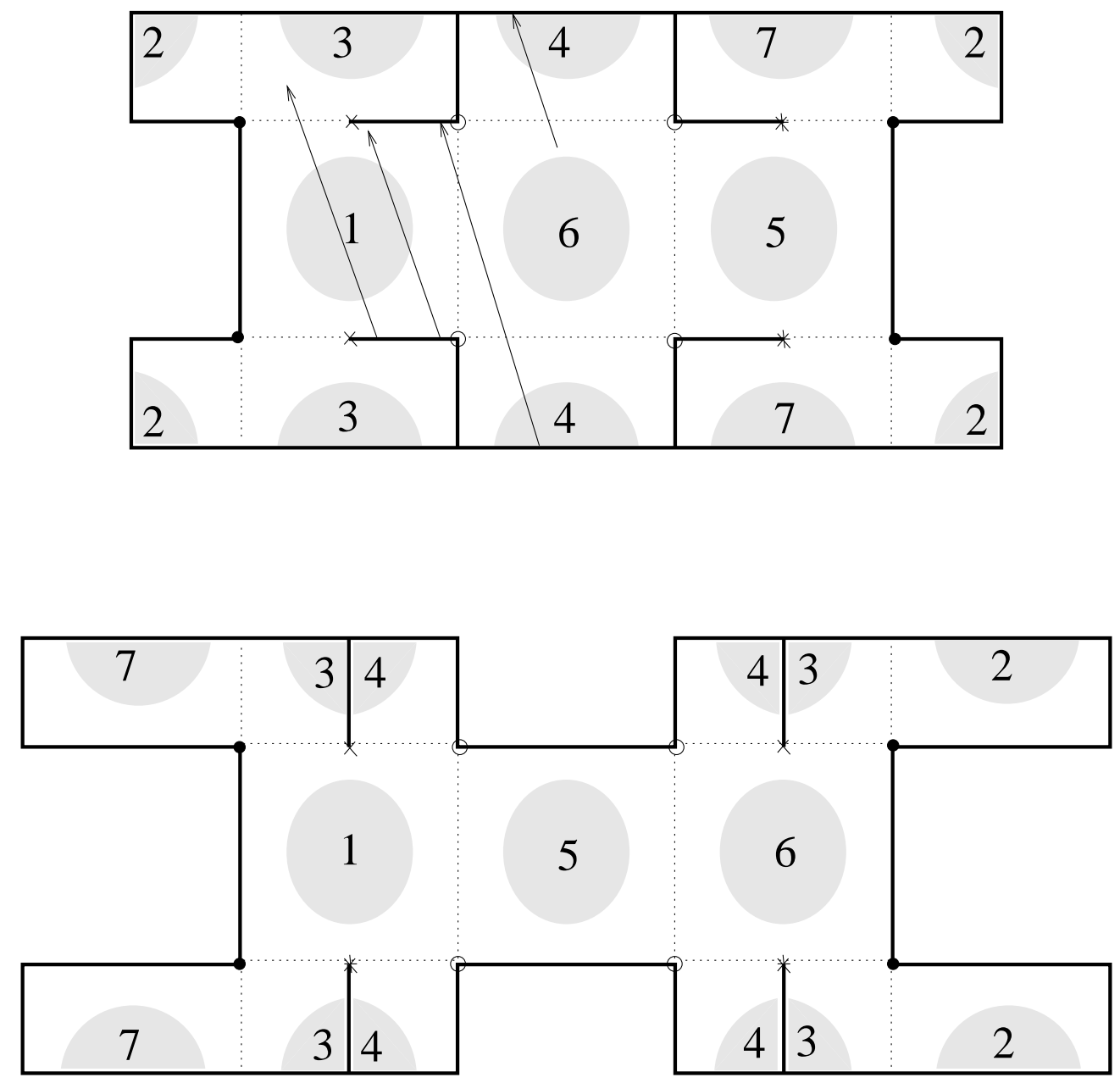

Figure 7 The pair $7_{3}$ of isospectral billiards with a rectangular base shape unfolded to a translation surface (i.e. flat billiard with opposite sides identified).

the first translation surface, the matrix specifying which tile is on the right of which is

$$
A^{(1)}=\left(\begin{array}{lllllll}
0 & 0 & 0 & 0 & 0 & 1 & 0 \\
0 & 0 & 1 & 0 & 0 & 0 & 0 \\
0 & 0 & 0 & 0 & 0 & 0 & 1 \\
0 & 0 & 0 & 1 & 0 & 0 & 0 \\
1 & 0 & 0 & 0 & 0 & 0 & 0 \\
0 & 0 & 0 & 0 & 1 & 0 & 0 \\
0 & 1 & 0 & 0 & 0 & 0 & 0
\end{array}\right)
$$

(tile 6 is on the right of tile 1 , therefore $A_{1,6}^{(1)}=1$, and so on). In a similar way, matrices 
$B^{(\nu)}, 1 \leq \nu \leq 6$, can be defined for the second translation surface. Now suppose there exists a matrix $T$ such that

$$
\forall i, 1 \leq i \leq 6, A^{(i)} T=T B^{(i)}
$$

Such a matrix provides a mapping between the two translation surfaces. It is called a "transplantation matrix". Then it is easy to show that for any given eigenstate $\phi$ of the first translation surface we can construct an eigenstate $\psi$ for the second translation surface, defined by Eq. (6). Obviously Helmholtz equation (3) is satisfied since it is linear. To prove isospectrality we only have to check for continuity properties at each edge. Suppose tiles $i$ and $j$ are glued together by one of their edges. There exists a $\nu, 1 \leq \nu \leq 6$, such that $A_{i j}^{(\nu)}=1$ ( $\nu$ is the label of the edge between tiles $i$ and $j$ ). It is such that

$$
|\psi(a, j)\rangle=\sum_{k} A_{i k}^{(\nu)}|\psi(a, k)\rangle
$$

where $|\psi(a, i)\rangle$ is the 7-dimensional vector whose components are $\psi(a, i), 1 \leq i \leq 7, a$ belonging to the edge between $i$ and $j$. To prove the continuity of $\psi$ between tiles $i$ and $j$, we have to show that the quantity

$$
\begin{aligned}
\mathcal{C} & =|\psi(a, i)\rangle-|\psi(a, j)\rangle \\
& =|\psi(a, i)\rangle-\sum_{k} A_{i k}^{(\nu)}|\psi(a, k)\rangle
\end{aligned}
$$

is equal to zero. Using Eq. 6, (11) becomes

$$
\mathcal{C}=\sum_{k} T_{i k}|\phi(a, k)\rangle-\sum_{k, k^{\prime}} A_{i k}^{(\nu)} T_{k k^{\prime}}\left|\phi\left(a, k^{\prime}\right)\right\rangle
$$

The sum over $k$ on the right-hand side yields a term $\left(A^{(\nu)} T\right)_{i k^{\prime}}$. By the commutation relation (9), it is equal to $\left(T B^{(\nu)}\right)_{i k^{\prime}}$, which gives

$$
\mathcal{C}=\sum_{k} T_{i k}\left(|\phi(a, k)\rangle-\sum_{k^{\prime}} B_{k k^{\prime}}^{(\nu)}\left|\phi\left(a, k^{\prime}\right)\right\rangle\right) .
$$

Now the continuity of the function $\phi$ ensures that the terms between brackets all vanish. 
Thus $\mathcal{C}=0$, and continuity is proven.

The proof rests entirely on the existence of a transplantation matrix $T$ satisfying the commutation properties (9). It turns out that such a matrix exists. One can check that given the matrix

$$
T=\left(\begin{array}{lllllll}
1 & 0 & 0 & 1 & 0 & 0 & 1 \\
0 & 1 & 0 & 0 & 1 & 0 & 1 \\
0 & 0 & 1 & 0 & 0 & 1 & 1 \\
1 & 0 & 0 & 0 & 1 & 1 & 0 \\
0 & 1 & 0 & 1 & 0 & 1 & 0 \\
0 & 0 & 1 & 1 & 1 & 0 & 0 \\
1 & 1 & 1 & 0 & 0 & 0 & 0
\end{array}\right),
$$

equations (9) are fulfilled for all $\nu, 1 \leq \nu \leq 6$. Thus the proof of isospectrality is completed. We will come back later in section VII on this transplantation proof of isospectrality.

The natural question is to know how one can find a suitable matrix $T$ verifying all commutation equations (9), and (to start with) how the matrices $A^{(\nu)}, B^{(\nu)}$ are obtained. Historically these matrices were obtained by the construction of Sunada triples, as will be explained in section III.3. In fact it turns out that the matrix $T$ is just the incidence matrix of the graph associated to a certain finite projective space (the Fano plane in our example), as will be explained in detail in section VII. 


\section{FURTHER EXAMPLES IN HIGHER DIMENSIONS}

In (Milnor, 1964), J. Milnor showed that from two nonisometric lattices of rank 16 in $\mathbb{R}^{16}$ discovered by Witt in (Witt, 1941), one can construct a pair of flat tori which have the same spectrum of eigenvalues. (All relevant terms will be defined below.)

In this section, we will describe a simple criterion for the construction of nonisometric flat tori with the same eigenvalues for the Laplace operator, from certain lattices (which was used by Milnor for the particular case mentioned in the beginning of this section), and then we construct, for each integer $n \geq 17$, a pair of lattices of rank $n$ in $\mathbb{R}^{n}$ which match the criterion. Furthermore, we describe powerful results of S. Wolpert and M. Kneser on the moduli space of flat tori.

\section{A. Lattices and flat tori}

A lattice (that is, a discrete additive subgroup) can be prescribed as $A \mathbb{Z}^{n}$ with $A$ a fixed matrix. For example, put

$$
A=\left(\begin{array}{ll}
1 & 0 \\
1 & 1
\end{array}\right)
$$

then the lattice $A \mathbb{Z}^{2}$ consists of the points of the form

$$
a(1,1)+b(0,1), \quad a, b \in \mathbb{Z} .
$$

An $n$-dimensional (flat) torus $T$ is $\mathbb{R}^{n}$ factored by a lattice $\mathbf{L}=A \mathbb{Z}^{n}$ with $A \in \mathbf{G L}(n, \mathbb{R})$. So the torus is determined by identifying points which differ by an element of the lattice.

If we go back to the planar example of above, the torus topologically is a doughnut - one may see this by cutting out the parallelogram determined by $(1,1)$ and $(0,1)$, and then gluing opposite sides together.

The metric structure of $\mathbb{R}^{n}$ projects to $T$, and volume $(T)=|\operatorname{det} A| ; T$ carries a Laplace 
operator

$$
\Delta=-\sum_{i} \partial^{2} / \partial x_{i}^{2}
$$

the projection of the Laplacian of $\mathbb{R}^{n}$. The lengths of closed geodesics of $T$ are given as $\|a\|$ for $a$ arbitrary in $\mathbf{L},\|\cdot\|$ being the Euclidean norm.

Let $P$ be a symmetric matrix which defines a quadratic form on $\mathbb{R}^{n}$. The spectrum of $P$ is defined to be the sequence (with multiplicities) of values $\gamma=N^{T} P N$ for $N \in \mathbb{Z}^{n}$. The sequence of squares of lengths of closed geodesics of $\mathbb{R}^{n} / A \mathbb{Z}^{n}$ is the spectrum of $A^{T} A=Q$; the sequence of eigenvalues is the spectrum of $4 \pi^{2}\left(A^{-1}\right)\left(A^{-1}\right)^{T}=4 \pi^{2} Q^{-1}$. The Jacobi inversion formula yields for positive $\tau$,

$$
\sum_{N \in \mathbb{Z}^{n}} \exp \left(-4 \pi^{2} \tau N^{T} Q^{-1} N\right)=\frac{\operatorname{volume}(T)}{(4 \pi \tau)^{n / 2}} \sum_{M \in \mathbb{Z}^{n}} \exp \left(\frac{-1}{4 \tau} M^{T} Q M\right) .
$$

We now explain the way in which $\mathbf{O}(n, \mathbb{R}) \backslash \mathbf{G L}(n, \mathbb{R}) / \mathbf{G L}(n, \mathbb{Z})$ is the moduli space of flat tori. Here, $\mathbf{O}(n, \mathbb{R})$ is the orthogonal group in $n$ dimensions. To $A \in \mathbf{G L}(n, \mathbb{R})$ is associated the lattice $A \mathbb{Z}^{n}$. The tori $\mathbb{R}^{n} / A \mathbb{Z}^{n}$ and $\mathbb{R}^{n} / B \mathbb{Z}^{n}$ are isometric if and only if $A \mathbb{Z}^{n}$ and $B \mathbb{Z}^{n}$ are isometric by left multiplication by an element of $\mathbf{O}(n, \mathbb{R})$. The matrices $A$ and $B$ are associated to the same lattice if and only if they are equivalent by multiplication on the right by an element of $\mathbf{G L}(n, \mathbb{R})$. So the tori $\mathbb{R}^{n} / A \mathbb{Z}^{n}$ and $\mathbb{R}^{n} / B \mathbb{Z}^{n}$ are isometric if and only if $A$ and $B$ are equivalent in

$$
\mathbf{O}(n, \mathbb{R}) \backslash \mathbf{G L}(n, \mathbb{R}) / \mathbf{G L}(n, \mathbb{Z}) .
$$

Denote the space of positive definite symmetric $n \times n$-matrices by $\wp(n, \mathbb{R})$, and observe that the map

$$
A \in \mathbf{G L}(n, \mathbb{R}) \mapsto A^{T} A \in \wp(n, \mathbb{R})
$$

determines a bijection from $\mathbf{O}(n, \mathbb{R}) \backslash \mathbf{G L}(n, \mathbb{R})$ to $\wp(n, \mathbb{R})$. 


\section{B. Construction of examples}

CRITERION. If $\mathbf{L}$ is a lattice of $\mathbb{R}^{n}, \mathbf{L}^{*}$ denotes its dual lattice, which consists of all $y \in \mathbb{R}^{n}$ for which $\langle x, y\rangle \in \mathbb{Z}$ for all $x \in \mathbf{L}$; here, $\langle\cdot, \cdot\rangle$ is the usual scalar product on $\mathbb{R}^{n} \times \mathbb{R}^{n}$. Clearly, $\left(\mathbf{L}^{*}\right)^{*}=\mathbf{L}$, and two lattices $\mathbf{L}$ and $\mathbf{L}^{\prime}$ are isometric if and only if $\mathbf{L}^{*}$ and $\mathbf{L}^{\prime *}$ are.

Recall that two flat tori of the form $\mathbb{R}^{n} / \mathbf{L}_{i}, i \in\{1,2\}$, are isometric if the lattices $\mathbf{L}_{1}$ and $\mathbf{L}_{2}$ are isometric.

We start by making the following observation.

Theorem II.1 Let $\mathbf{L}_{1}$ and $\mathbf{L}_{2}$ be two nonisometric lattices of rank $n$ in $\mathbb{R}^{n}, n \geq 2$, and suppose that for each $r>0$ in $\mathbb{R}$, the ball of radius $r$ about the origin contains the same number of points of $\mathbf{L}_{1}$ and $\mathbf{L}_{2}$. Then the flat tori $\mathbb{R}^{n} / \mathbf{L}_{1}^{*}$ and $\mathbb{R}^{n} / \mathbf{L}_{2}^{*}$ are nonisometric while having the same spectrum for the Laplace operator.

Proof. Suppose $x \neq \mathbf{0}$ is an element of $\mathbf{L}_{1}$ of length $\alpha$. Then there is an $\alpha^{\prime}<\alpha$ such that the ball of radius $\alpha^{\prime}$ centered at $\mathbf{0}$ contains all elements of $\mathbf{L}_{1}$ with length strictly smaller than $\alpha$ (since $\mathbf{L}_{1}$ is discrete). Since this ball contains as many elements of $\mathbf{L}_{2}$ as of $\mathbf{L}_{1}$, it follows easily that $\mathbf{L}_{2}$ also contains vectors of length $\alpha$.

Each element $z \in \mathbf{L}_{i}, i \in\{1,2\}$, determines an eigenfunction $f(x)=e^{2 \pi\langle x, z\rangle} i$ for the Laplace operator on $\mathbb{R}^{n} / \mathbf{L}_{i}^{*}$, with corresponding eigenvalue $\lambda=(2 \pi)^{2}\langle z, z\rangle$, so the number of eigenvalues less than or equal to $(2 \pi r)^{2}$ is equal to the number of points of $\mathbf{L}_{i}$ contained in the ball centered at $\mathbf{0}$ with radius $r$.

We conclude that $\mathbb{R}^{n} / \mathbf{L}_{1}^{*}$ and $\mathbb{R}^{n} / \mathbf{L}_{2}^{*}$ have the same spectrum of eigenvalues, while being not isometric.

Now consider the nonisometric lattices $\mathbf{L}_{1}^{16}$ and $\mathbf{L}_{2}^{16}$ of rank 16 in $\mathbb{R}^{16}$ as described by Witt in (Witt, 1941). These lattices satisfy the condition of Theorem II.1 (Witt, 1941, p.324). Now embed $\mathbb{R}^{16}$ in $\mathbb{R}^{17}$ in the canonical way. Denote the coordinate axes of the latter by $X_{1}, X_{2}, \ldots, X_{17}$, such that $\left\langle X_{1}, X_{2}, \ldots, X_{16}\right\rangle=\mathbb{R}^{16}$. Suppose $\ell \neq \mathbf{0}$ is a vector on the $X_{17^{-}}$ axis which has length strictly smaller than any non-zero vector of $\mathbf{L}_{1}$ (and $\mathbf{L}_{2}$ ). Define two new lattices $\mathbf{L}_{i}^{17}$ (of rank 17) generated by $\mathbf{L}_{i}^{16}$ and $\ell, i=1$, 2. Since $X_{17} \perp \mathbb{R}^{16}$, it follows easily that for any $r>0$, the ball centered at the origin with radius $r$ contains the same number of elements of $\mathbf{L}_{1}^{17}$ as of $\mathbf{L}_{2}^{17}$. One observes that these lattices are nonisometric. 
Whence, by Theorem II.1, we obtain two nonisometric flat tori $\mathbb{R}^{17} / \mathbf{L}_{i}^{17^{*}}, i=1,2$, which have the same spectrum of eigenvalues for the Laplace operator.

Inductively, we can now define, in a similar way, the nonisometric lattices $\mathbf{L}_{1}^{n}$ and $\mathbf{L}_{2}^{n}$ of rank $n, n \geq 17$, satisfying the condition of Theorem II.1, and thus leading to nonisometric flat tori $\mathbb{R}^{n} / \mathbf{L}_{i}^{n *}, i=1,2$, which have the same spectrum of eigenvalues for the Laplace operator.

Milnor's Construction. By using the Witt nonisometric lattices in $\mathbb{R}^{16}$ (Witt, 1941), John Milnor (Milnor, 1964) essentially used the aforementioned criterion to construct the first example of nonisometric isospectral flat tori.

\section{The eigenvalue spectrum as moduli for flat tori}

We now quickly browse through some interesting results on the eigenvalue spectrum for flat tori.

We already saw that there exist nonisometric isospectral flat tori. A natural question is now how such tori are distributed.

Theorem II.2 (S. Wolpert (Wolpert, 1978)) Let $T_{s}$ be a continuous family of isospectral tori defined for $s \in[0,1]$. The tori $T_{s}, s \in[0,1]$, are isometric.

A very interesting (unpublished) result by M. Kneser is the following (see (Wolpert, 1978) for a proof):

Theorem II.3 (M. Kneser) The total number of nonisometric tori with a given eigenvalue spectrum is finite.

So given an eigenvalue spectrum of some torus, only a finite number of nonisometric tori can be isospectral to it.

The following result is rather technical. 
Theorem II.4 (S. Wolpert (Wolpert, 1978)) There is a properly discontinuous group $G_{n}$ acting on $\wp(n, \mathbb{R})$ containing the transformation group induced by the $\mathbf{G L}(n, \mathbb{Z})$ action

$$
S \mapsto A[\mathcal{Z}]
$$

where $S \in \wp(n, \mathbb{R})$ and $\mathcal{Z} \in \mathbf{G L}(n, \mathbb{Z})$. Given $P, S \in \wp(n, \mathbb{R})$ with the same spectrum either $g(P)=S$ for some $g \in G_{n}$, or $P, S \in \mathfrak{V}_{n}$, where the latter is a subvariety of $\wp(n, \mathbb{R})$. Moreover,

(i) $\mathfrak{V}_{n}=\left\{Q \in \wp(n, \mathbb{R}) \| \operatorname{spec}(Q)=\operatorname{spec}(R), R \in \wp(n, \mathbb{R})\right.$ with $R \neq g(Q)$ for all $\left.g \in G_{n}\right\}$, and

(ii) $\mathfrak{V}_{n}$ is the intersection of $\wp(n, \mathbb{R})$ and a countable union of subspaces of $\mathbb{R}^{m}$ for some $m$.

In this section we have seen that is essentially "easy" to construct (nonisometric) isospectral flat tori. The Milnor example was exhibited in 1964. But it has taken about 30 years to find counter examples to Kac's question in the real plane ... 


\section{SUNADA THEORY}

We review some basic notions of group theory. We will be slightly more elaborate than just restricting ourselves to the setting we (strictly) need. Then we will introduce Sunada Theory.

\section{A. Permutations}

We denote permutation action exponentially (the image of an element $x$ by the permutation $g$ is $x^{g}$ ) and let elements act on the right. We denote the identity element of a group by id or 1, if no special symbol has been introduced for it before. A group $G$ without its identity id is denoted $G^{\times}$. The number of elements of a group $G$ is denoted by $|G|$. We write the action of a group on a set at the right, as an exponent, and as such a permutation group $(G, X)$ is a pair consisting of a group $G$ and a set $X$ such that each element $g$ of $G$ defines a permutation $g: X \rightarrow X$ of $X$, and the permutation defined by $g h, g, h \in G$, is given by $g h: X \rightarrow X: x \mapsto\left(x^{g}\right)^{h}$.

Finally, an involution in a group is an element of order 2.

\section{B. Commutator notions}

The conjugate of $g$ by $h$ is $g^{h}=h^{-1} g h$. Let $H$ be a group. The commutator of two group elements $g, h$ is equal to $[g, h]=g^{-1} h^{-1} g h$. The commutator of two subsets $A$ and $B$ of a group $G$ is the subgroup $[A, B]$ generated by all elements $[a, b]$, with $a \in A$ and $b \in B$. The commutator subgroup of $G$ is $[G, G]$, also denoted by $G^{\prime}$. Two subgroups $A$ and $B$ centralize each other if $[A, B]=\{\mathbf{i d}\}$. The subgroup $A$ normalizes $B$ if $B^{a}=B$, for all $a \in A$, which is equivalent with $[A, B]$ being a subgroup of $B$. If $A$ and $B$ are two subgroups of the group $G$, then they are conjugate(d) if there is an element $g$ of $G$ such that $A^{g}=B$. The subgroup $A$ of $G$ is (a) normal (subgroup) in (of) $G$ if $A^{g}=A$ for all $g \in G$. In such a case, we write $A \unlhd G$. If $A \neq G$, we also write $A \triangleleft G$.

Inductively, we define the $n$th central derivative $[G, G]_{[n]}$ of a group $G$ as $\left[G,[G, G]_{[n-1]}\right]$, and the $n$th normal derivative $[G, G]_{(n)}$ as $\left[[G, G]_{(n-1)},[G, G]_{(n-1)}\right]$. For $n=0$, the 0th 
central and normal derivative are by definition equal to $G$ itself. If, for some natural number $n,[G, G]_{(n)}=\{\mathbf{i d}\}$, and $[G, G]_{(n-1)} \neq\{\mathbf{i d}\}$, then we say that $G$ is solvable (soluble) of length $n$. If $[G, G]_{[n]}=\{\mathbf{i d}\}$ and $[G, G]_{[n-1]} \neq\{\mathbf{i d}\}$, then we say that $G$ is nilpotent of class $n$. The center of a group is the set of elements that commute with every other element, i.e., $Z(G)=\{z \in G \|[z, g]=\mathrm{id}, \forall g \in G\}$. Clearly, if a group $G$ is nilpotent of class $n$, then the $(n-1)$ th central derivative is a nontrivial subgroup of $Z(G)$.

A group $G$ is the central product of its subgroups $A$ and $B$ if $A B=G, A \cap B$ is contained in the center of $G$, and $A$ and $B$ centralize each other. Sometimes we write $G=A \circ B$ in such a case.

A group $G$ is called perfect if $G=[G, G]=G^{\prime}$.

Let $R$ be a finite group. The Frattini group $\phi(R)$ of $R$ is the intersection of all proper maximal subgroups, or is $R$ if $R$ has no such subgroups.

\section{C. $p$-Groups and extra-special groups}

For a prime number $p$, a $p$-group is a group of order $p^{n}$, for some natural number $n \neq 0$. A Sylow $p$-subgroup of a finite group $G$ is a $p$-subgroup of some order $p^{n}$ such that $p^{n+1}$ does not divide $|G|$.

A $p$-group $P$ is special if either $[P, P]=Z(P)=\phi(P)$ is elementary abelian or $P$ itself is. (A group is elementary abelian if it is abelian, and if there exists a prime $p$ so that each of its nonidentity elements has order $p$.) Note that $P /[P, P]$ is elementary abelian in that case. So we have the exact sequence

$$
\mathbf{1} \mapsto[P, P] \mapsto P \mapsto V(n, p) \mapsto \mathbf{1}
$$

where $V(n, p)$ is the $n$-dimensional vector space over $\mathbb{F}_{p}$ and $|P|=p^{n}|[P, P]|$. So

$$
P /[P, P] \cong V(n, p)
$$


the latter now seen as its additive group.

If $|Z(P)|=|[P, P]|=|\phi(P)|=p, P$ is called extra-special.

We now present a classification for extra-special groups which depends on the knowledge of the nonabelian $p$-groups of order $p^{3}$.

There are four nonabelian $p$-groups of order $p^{3}$ - see (Gorenstein, 1980). First of all, we have $M=M(p)$ :

$$
M(p)=\left\langle x, y, z \| x^{p}=y^{p}=z^{p}=\mathbf{1},[x, z]=[y, z]=\mathbf{1},[x, y]=z\right\rangle .
$$

(Note that this is the general Heisenberg group of order $p^{3}$ which we will encounter later on.) Next, define

$$
M_{3}(p)=\left\langle x, y \| x^{p^{2}}=y^{p}=\mathbf{1}, x^{y}=x^{p+1}\right\rangle .
$$

Finally, we have the dihedral group $D$ of order 8 and the generalized quaternion group $Q$ of order 8 .

Theorem III.1 ((Gordon, 1986)) An extra-special p-group $P$ is the central product of $r \geq 1$ nonabelian subgroups of order $p^{3}$. Moreover, we have

(1) If $p$ is odd, $P$ is isomorphic to $N^{k} M^{r-k}$, while if $p=2, P$ is isomorphic to $D^{k} Q^{r-k}$ for some $k$. In either case, $|P|=p^{2 r+1}$.

(2) If $p$ is odd and $k \geq 1, N^{k} M^{r-k}$ is isomorphic to $N M^{r-1}$, the groups $M^{r}$ and $N M^{r-1}$ are not isomorphic and $M^{r}$ is of exponent $p$.

(3) If $p=2$, then $D^{k} Q^{r-k}$ is isomorphic to $D Q^{r-1}$ if $k$ is odd and to $Q^{r}$ if $k$ is even, and the groups $Q^{r}$ and $D Q^{r-1}$ are not isomorphic.

\section{Finite simple groups}

A group is simple if it does not contain nontrivial normal subgroups. 
The finite simple groups are often regarded as the elementary particles in Finite Group Theory. Before explaining this a bit more precisely, recall that a composition series of a group $G$ is a normal series

$$
1=H_{0} \triangleleft H_{1} \triangleleft \cdots \triangleleft H_{n}=G,
$$

such that each $H_{i}$ is a maximal normal subgroup of $H_{i+1}$. Equivalently, a composition series is a normal series such that each factor group $H_{i+1} / H_{i}$ is simple. The factor groups are called composition factors.

A normal series is a composition series if and only if it is of maximal length. That is, there are no additional subgroups which can be "inserted" into a composition series. The length $n$ of the series is called the composition length.

If a composition series exists for a group $G$, then any normal series of $G$ can be refined to a composition series. Furthermore, every finite group has a composition series.

A group may have more than one composition series. However, the Jordan-Hölder theorem states that any two composition series of a given group are equivalent.

The classification of finite simple groups (see (Solomon, 2001) for a survey) states that

Every finite simple group is cyclic, or alternating, or is contained in one of 16 families of groups of Lie type (including the Tits group, which strictly speaking is not of Lie type), or one of 26 sporadic groups.

In an appendix to this paper we list the finite simple groups, with some additional information on the nomenclature.

In this review, we will encounter several aspects of certain simple groups in the construction theory of counter examples to Kac's initial question.

\section{E. Sunada Theory}

Recall that a number field is a finite, algebraic field extension of $\mathbb{Q}$. A standard example is $\mathbb{Q}(\sqrt{2})$. Let $\mathbb{K}$ be as such. The (Dedekind) zeta function $\zeta_{\mathbb{K}}(s)$ (associated to $\mathbb{K}$ ), $s$ being a complex variable, is 


$$
\sum_{I}\left(N_{Q}^{\mathbb{K}}(I)\right)^{-s}
$$

taken over all ideals $I$ of the ring of integers $O_{\mathbb{K}}$ of $\mathbb{K}, I \neq\{0\}$. Note that $N_{Q}^{\mathbb{K}}(I)$ denotes the norm of $I$ (to $\mathbb{Q}$ ), equal to $\left|O_{\mathbb{K}} / I\right|$.

Let $\mathbb{K}$ be an algebraic number field of degree $n$. Let $p$ be a rational prime. Let $P_{1}, \ldots, P_{g}$ be the prime ideals of $O_{\mathbb{K}}$ lying above $p$. Then

$$
\langle p\rangle=\prod_{i=1}^{g} P_{i}^{e_{i}}
$$

where

$$
e_{i}=e_{\mathbb{K}}\left(P_{i}\right)
$$

If $e_{i}>1$ for some $i \in\{1, \ldots, g\}$, then $p$ is said to be ramified in $\mathbb{K}$. If $e_{i}=1$ for all $i, p$ is unramified in $\mathbb{K}$.

Let $\mathbb{K}=\mathbb{Q}(\theta)$ be as above, that is, an algebraic number field of degree $n$. Suppose $\theta_{1}, \theta_{2}, \ldots, \theta_{n}$ are the conjugates of $\theta$ over $\mathbb{Q}$. If

$$
\mathbb{Q}\left(\theta_{1}\right)=\cdots=\mathbb{Q}\left(\theta_{n}\right)=\mathbb{K}
$$

then $\mathbb{K}$ is a Galois extension of $\mathbb{Q}$.

A number-theoretic exercise which asks for non-isomorphic number fields $\mathbb{K}_{1}$ and $\mathbb{K}_{2}$ with the same zeta function has the following answer:

Theorem III.2 (K. Komatsu (Komatsu, 1976)) Let $\mathbb{K}$ be a finite Galois extension of $\mathbb{Q}$ with Galois group $G=\operatorname{Gal}(\mathbb{K} / \mathbb{Q})$, and let $\mathbb{K}_{1}$ and $\mathbb{K}_{2}$ be subfields of $\mathbb{K}$ corresponding to subgroups $G_{1}$ and $G_{2}$ of $G$, respectively. Then the following conditions are equivalent:

(i) Each conjugacy class of $G$ meets $G_{1}$ and $G_{2}$ in the same number of elements; 
(ii) The same primes $p$ are ramified in $\mathbb{K}_{1}$ and $\mathbb{K}_{2}$ and for the unramified $p$ the decomposition of $p$ in $\mathbb{K}_{1}$ and $\mathbb{K}_{2}$ is the same;

(iii) The zeta functions of $\mathbb{K}_{1}$ and $\mathbb{K}_{2}$ are the same.

In particular, if $G_{1}$ and $G_{2}$ are not conjugate in $G$, then $\mathbb{K}_{1}$ and $\mathbb{K}_{2}$ are not isomorphic while having the same zeta function. It should be noted that several such triples $\left(G, G_{1}, G_{2}\right)$ are known - see the examples paragraph further in this section.

Any group triples $\left(G, G_{1}, G_{2}\right)$ satisfying Theorem III.2(i) are said to satisfy "Property (*)".

\section{F. Sunada's theorem and a trace formula}

Sunada's idea was to establish a counterpart of this theorem for Riemannian geometry. In that context, there also is an analogue for the zeta function. For $\mathcal{M}$ a Riemannian manifold, one defines

$$
\zeta_{\mathfrak{M}}(s)=\sum_{i=1}^{\infty} \lambda_{i}^{-s}, \quad \mathfrak{R e}(s) \geq 0
$$

where

$$
0<\lambda_{1} \leq \lambda_{2} \leq \cdots
$$

are the non-zero eigenvalues of the Laplacian for $\mathfrak{M}$.

The function $\zeta_{\mathfrak{M}}$ has an analytic continuation to the whole plane, and it is well-known that $\zeta_{\mathfrak{M}_{1}}(s)=\zeta_{\mathfrak{M}_{2}}(s)$ if and only if $\mathfrak{M}_{1}$ and $\mathfrak{M}_{2}$ are isospectral.

The following theorem gives sufficient conditions for two manifolds to have the same zeta function.

Theorem III.3 (T. Sunada (Sunada, 1985)) Let $\pi: \mathfrak{M} \mapsto \mathfrak{M}_{0}$ be a normal finite Riemannian covering with covering transformation group $G$, and let $\pi_{1}: \mathfrak{M}_{1} \mapsto \mathfrak{M}_{0}$ and $\pi_{2}: \mathfrak{M}_{2} \mapsto \mathfrak{M}_{0}$ be the coverings corresponding to the subgroups $H_{1}$ and $H_{2}$ of $G$, respectively. If the triplet $\left(G, H_{1}, H_{2}\right)$ satisfies Property (*), then the zeta functions $\zeta_{\mathfrak{M}_{1}}(s)$ and $\zeta_{\mathfrak{M}_{2}}(s)$ are identical. 
The proof of the latter theorem makes use of an interesting trace formula, which we present now.

Let $V$ be a Hilbert space on which a finite group $G$ acts as unitary transformations and let $A: V \mapsto V$ be a self-adjoint operator of trace class such that $A$ commutes with the $G$-action. For a subgroup $H$ of $G$, denote by $V^{H}$ the subspace of $H$-invariant vectors.

Trace Formula. The restriction of $A$ to the subspace $V^{G}$ is also of trace class, and

$$
\operatorname{tr}\left(\left.A\right|_{V^{G}}\right)=\sum_{[g] \in[G]}\left(\left|G_{g}\right|\right)^{-1} \operatorname{tr}(g A)
$$

where $[G]=\{[g]\}$ is the set of conjugacy classes in $G$ and $G_{g}$ is the centralizer of $g$ in $G$.

If the triplet $\left(G, G_{1}, G_{2}\right)$ satisfies Property (*), then

$$
\operatorname{tr}\left(\left.A\right|_{V^{G_{1}}}\right)=\operatorname{tr}\left(\left.A\right|_{V^{G_{2}}}\right)
$$

Even if $G_{1}$ and $G_{2}$ are not conjugate, the manifolds $\mathfrak{M}_{1}$ and $\mathfrak{M}_{2}$ could possibly be isometric.

Theorem III.4 (T. Sunada (Sunada, 1985)) There exist finite coverings $\pi_{1}: \mathfrak{M}_{1} \mapsto \mathfrak{M}_{0}$ and $\pi_{2}: \mathfrak{M}_{2} \mapsto \mathfrak{M}_{0}$ of Riemann surfaces with genus $\geq 2$ such that for a generic metric $g_{0}$ on $\mathfrak{M}_{0}$, the surfaces $\left(\mathfrak{M}_{1}, \pi_{1}^{*} g_{0}\right)$ and $\left(\mathfrak{M}_{2}, \pi_{2}^{*} g_{0}\right)$ are isospectral, but not isometric.

Sunada's Theorem allows us to construct isospectral pairs provided we find triples $\left(G, G_{1}, G_{2}\right)$ satisfying Property (*).

Now we give examples of such triples.

\section{G. Property (*): examples}

Example 1 - see I. Gerst (Gerst, 1970). Let $G$ be the semidirect product $\mathbb{Z} / 8 \mathbb{Z}^{\times} \times \mathbb{Z} / 8 \mathbb{Z}$, and define $G_{1}$ and $G_{2}$ by

$$
G_{1}=\{(1,0),(3,0),(5,0),(7,0)\}, \quad G_{2}=\{(1,0),(3,4),(5,4),(7,0)\} .
$$


Example 2 - see F. Gassman (Gassmann, 1926). Let $G=\mathbf{S}_{6}$ be the symmetric group on 6 letters $\{a, b, c, d, e, f\}$. Put

$$
G_{1}=\{\mathbf{1},(a b)(c d),(a c)(b d),(a d)(b c)\}
$$

and

$$
G_{2}=\{\mathbf{1},(a b)(c d),(a b)(e f),(c d)(e f)\}
$$

Example 3 - see K. Komatsu (Komatsu, 1976). Let $G_{2}$ and $G_{2}$ be two finite groups with the same order, and suppose that their exponents (= the least common multiples of the orders of their elements) both equal the same odd prime $p$. Put $\left|G_{1}\right|=\left|G_{2}\right|=p^{h}$ for $h \in \mathbb{N}^{\times}$, and embed $G_{1}$ and $G_{2}$ in the symmetric group $\mathbf{S}_{p^{h}}$ on $p^{h}$ letters by their left action on themselves. For a conjugacy class $[g]$ corresponding to the partition

$$
\left|\mathbf{S}_{p^{h}}\right|=p^{h} !=p+p+\cdots+p
$$

we have

$$
\left|\left([g] \cap G_{1}\right)\right|=p^{h}-1=\left|\left([g] \cap G_{2}\right)\right|,
$$

while $\left|\left([g] \cap G_{i}\right)\right|=0$ otherwise.

Concretely, let $G_{1}=(\mathbb{Z} / p \mathbb{Z})^{3}$, and let $G_{2}$ be the group

$$
G_{2}=\left\langle a, b \| a^{p}=b^{p}=[a, b]^{p}=\mathbf{1}, a[a, b]=[a, b] b, b[a, b]=[a, b] b\right\rangle,
$$

that is, $G_{2}$ is the extra-special group of order $p^{3}$. Then $\left(\mathbf{S}_{p^{3}}, G_{1}, G_{2}\right)$ verify Property (*).

One can in fact generalize Komatsu's example by defining the following group. The general Heisenberg group $\mathbf{H}_{n}$ of dimension $2 n+1$ over $\mathbb{F}_{q}$, with $n$ a natural number, is the group of square $(n+2) \times(n+2)$-matrices with entries in $\mathbb{F}_{q}$, of the following form (and with the usual matrix multiplication): 


$$
\left(\begin{array}{ccc}
1 & \alpha & c \\
0 & \mathbf{I}_{n} & \beta^{T} \\
0 & 0 & 1
\end{array}\right)
$$

where $\alpha, \beta \in \mathbb{F}_{q}^{n}, c \in \mathbb{F}_{q}$ and with $\mathbf{I}_{n}$ being the $n \times n$-unit matrix. Let $\alpha, \alpha^{\prime}, \beta, \beta^{\prime} \in \mathbb{F}_{q}^{n}$ and $c, c^{\prime} \in \mathbb{F}_{q}$; then

$$
\left(\begin{array}{ccc}
1 & \alpha & c \\
0 & \mathbf{I}_{n} & \beta^{T} \\
0 & 0 & 1
\end{array}\right) \times\left(\begin{array}{ccc}
1 & \alpha^{\prime} & c^{\prime} \\
0 & \mathbf{I}_{n} & \beta^{\prime T} \\
0 & 0 & 1
\end{array}\right)=\left(\begin{array}{ccc}
1 & \alpha+\alpha^{\prime} & c+c^{\prime}+\left\langle\alpha, \beta^{\prime}\right\rangle \\
0 & \mathbf{I}_{n} & \beta+\beta^{\prime} \\
0 & 0 & 1
\end{array}\right)
$$

Here $\langle x, y\rangle$, with $x=\left(x_{1}, x_{2}, \ldots, x_{n}\right)$ and $y=\left(y_{1}, y_{2}, \ldots, y_{n}\right)$ elements of $\mathbb{F}_{q}^{n}$, denotes $x_{1} y_{1}+x_{2} y_{2}+\ldots+x_{n} y_{n}$.

The following properties hold for $\mathbf{H}_{n}$.

(i) $\mathbf{H}_{n}$ has exponent $p$ if $q=p^{h}$ with $p$ an odd prime; it has exponent 4 if $q$ is even.

(ii) The center of $\mathbf{H}_{n}$ is given by

$$
\left\{(0, c, 0) \| c \in \mathbb{F}_{q}\right\}
$$

(iii) $\mathbf{H}_{n}$ is nilpotent of class 2 .

Then similarly as above, $\left(\mathbf{S}_{p^{2 n+1}}, \mathbf{H}_{n},(\mathbb{Z} / p \mathbb{Z})^{2 n+1}\right)$ verifies Property (*).

Any finite group arises as the fundamental group of a compact smooth manifold of dimension 4. For a triplet $\left(G, G_{1}, G_{2}\right)$ of the type described in Example 3, we find a compact manifold $\mathfrak{M}_{0}$ with fundamental group $G$. Let $\mathfrak{M}$ be the universal covering of $\mathfrak{M}_{0}$. Then the quotients $\mathfrak{M}_{i}=\mathfrak{M} / G_{i}$ have non-isomorphic fundamental groups $G_{i}, i=1,2$. By Theorem III.3 the manifolds $\left(\mathfrak{M}_{1}, \pi_{1}^{*} g_{0}\right)$ and $\left(\mathfrak{M}_{2}, \pi_{2}^{*} g_{0}\right)$ are isospectral for any metric $g_{0}$ on $\mathfrak{M}_{0}$, but not isometric. 


\section{LIVSIC COHOMOLOGY}

In this section, we describe a connection between isospectrality and cohomology. We start with tersely introducing group cohomology.

\section{A. Group cohomology}

\section{Group modules}

Let $G$ be a group. A (left) $G$-module $M$ is an abelian group (written additively) on which $G$ acts as endomorphisms. In other words, a $G$-module is an abelian group $M$ together with a map

$$
G \times M \mapsto M, \quad(g, m) \mapsto g m
$$

such that for all $g, h \in G$ and $m, n \in M$ the following properties hold:

$$
\begin{gathered}
g(m+n)=g m+g n \\
(g h) m=g(h m) \\
1 m=m .
\end{gathered}
$$

Example. Let $G$ be a group. The module $M=\mathbb{Z}[G]$ with the action

$$
h\left(\sum_{g} n_{g} g\right)=\sum_{g} n_{g} h g
$$

is called the regular G-module.

Let $M$ be a $G$-module, and define the module of invariants $M^{G}$ as

$$
M^{G}=\{m \in M \| g m=m \text { for all } g \in G\} .
$$


$M^{G}$ is of course a submodule of $M$.

\section{The $n$-th cohomology group}

Let $A$ be a $G$-module and let $C^{n}(G, A)$ denote the set of functions of $n$ variables

$$
f: G \times G \times \cdots \times G \mapsto A
$$

into $A$. If $n=0, C^{0}(G, A)=\operatorname{Hom}(\mathbf{1}, A) \cong A$. The elements of $C^{n}(G, A)$ are $n$-cochains. Clearly, $C^{n}(G, A)$ is an abelian group with the usual addition and trivial element.

Now define homomorphisms $\delta=\delta_{n}: C^{n}(G, A) \mapsto C^{n+1}(G, A)$ as follows.

$$
\begin{gathered}
\delta_{n}(f)\left(x_{1}, \ldots, x_{n+1}\right)=x_{1} f\left(x_{2}, \ldots, x_{n+1}\right)+ \\
\sum_{i=1}^{n}(-1)^{i} f\left(x_{1}, \ldots, x_{i-1}, x_{i} x_{i+1}, \ldots, x_{n+1}\right) \\
+(-1)^{n+1} f\left(x_{1}, \ldots, x_{n}\right) .
\end{gathered}
$$

One can prove that $\delta_{n+1} \delta_{n}\left(C^{n}(G, A)\right)=0$ for all $n \in \mathbb{N}$. So the following sequence is a complex:

$$
A \mapsto^{\delta_{0}} \quad C^{1}(G, A) \mapsto^{\delta_{1}} \quad \ldots \mapsto^{\delta_{n-1}} \quad C^{n}(G, A) \mapsto^{\delta_{n}} \quad C^{n+1}(G, A) \mapsto^{\delta_{n+1}} \quad \ldots
$$

Now define the subgroups $Z^{n}(G, A)=\operatorname{ker} \delta_{n}$ and $B^{n}(G, A)=\operatorname{im} \delta_{n-1}$. For $n=0$ let $B^{0}(G, A)=0$. The elements of $Z^{n}(G, A)$ are the n-cocycles; the elements of $B^{n}(G, A)$ the $n$-coboundaries. Since $B^{n}(G, A)$ is a normal subgroup of $Z^{n}(G, A)$, factor groups can be formed. The $n$-th cohomology group of $G$ with coefficients in $A$ is then given by

$$
H^{n}(G, A)=Z^{n}(G, A) / B^{n}(G, A)=\operatorname{ker} \delta_{n} / \operatorname{im} \delta_{n-1} .
$$

For $n=0$ we have 


$$
H^{0}(G, A)=Z^{0}(G, A)=\{a \in A \| x a=a \text { for all } x \in G\}=A^{G},
$$

the module of invariants.

Example. Let $M$ be a $G$-module and regard $\mathbb{Z}$ as a trivial $G$-module. Then

$$
H^{0}(G, M)=M^{G} \cong \operatorname{Hom}_{G}(\mathbb{Z}, M)
$$

If $A$ is a $G$-module, then

$$
Z^{1}(G, A)=\{f: G \mapsto A \| f(x y)=x f(y)+f(x)\}
$$

and

$$
B^{1}(G, A)=\{f: G \mapsto A \| f(x)=x a-a \text { for some } a \in A\}
$$

The 1-cocycles are also called crossed homomorphisms of $G$ into $A$.

Proposition IV.1 Let $A$ be a G-module. Then there exists a bijection between $H^{1}(G, A)$ and the set of conjugacy classes of subgroups $H \leq G \ltimes A$ complementary to $A$, in which the conjugacy class of $G$ maps to zero. All the complements of $A$ in $G \ltimes A$ are conjugate if and only if $H^{1}(G, A)=0$.

The following proposition is known as "Hilbert's Satz 90" (which we present in the form of E. Noether's generalization):

Proposition IV.2 Let $L / K$ be a finite Galois extension with Galois group $G=\operatorname{Gal}(L / K)$. Then $H^{1}\left(G, L^{\times}\right)=\mathbf{1}$ and $H^{1}(G, L)=0$.

We mention the following result on the second cohomology group.

Theorem IV.3 Let $G$ be a group and $A$ an abelian group, and let $M$ denote the set of group extensions 


$$
0 \mapsto A \mapsto E \mapsto G \mapsto \mathbf{1}
$$

with a given $G$-module structure on $A$. Then there is a $1-1$ correspondence between the set of equivalence classes of extensions of $A$ by $G$ contained in $M$ with the elements of $H^{2}(G, A)$. The class of split extensions in $M$ corresponds to the class $[0] \in H^{2}(G, A)$.

For finite groups, one has the next theorem.

Theorem IV.4 Let $G$ be a finite group and $A$ be a G-module. Then every element of $H^{n}(G, A), n \in \mathbb{N}$, has a finite order which divides $|G|$. If $A$ is a finite G-module and $(|G|,|A|)=1$, then $H^{n}(G, A)=0$ for all $n \geq 1$. So any extension of $A$ by $G$ is split.

\section{B. Livsic's cohomological equation}

Let $(M, g)$ be a Riemannian manifold without boundaries. The length spectrum is the discrete set

$$
\operatorname{Lsp}(M, g)=\left\{L_{\gamma_{1}}<L_{\gamma_{2}}<\cdots\right\}
$$

of lengths of closed geodesics $\gamma_{j}$.

Denote by $\left(T^{*} M, \sum_{j} d x_{j} \wedge d \xi_{j}\right)$ the cotangent bundle of $M$ equipped with its natural symplectic form. Given the metric $g$, we define the metric Hamiltonian by

$$
H(x, \xi)=|\xi|=\sqrt{\sum_{i j=1}^{n+1} g^{i j}(x) \xi_{i} \xi_{j}},
$$

and define the energy surface to be the unit sphere bundle

$$
S^{*} M=\{(x, \xi) \||\xi|=1\} .
$$

The geodesic flow $G^{t}$ is the Hamiltonian flow 


$$
G^{t}=\exp t \Xi_{H}: T^{*} M \backslash 0 \mapsto T^{*} M \backslash 0,
$$

where $\Xi_{H}$ is the Hamiltonian vector field. Since it is homogeneous of degree 1 with respect to the dilatation $(x, \xi) \mapsto(x, r \xi), r>0$, one can restrict $G^{t}$ to $S^{*} M$. Its generator is also denoted by $\Xi$.

Livsic's cohomological problem asks whether a cocycle $f \in C^{\infty}\left(S^{*} M\right)$ satisfying

$$
\int_{\lambda} f d s=0
$$

for every closed geodesic of the metric $g$ is necessarily a coboundary $f=\Xi(g)$, where $\Xi$ is the generator of the geodesic flow $G^{t}$ and $g$ is a function with a certain degree of regularity. Under a deformation $g_{\epsilon}$ of a metric $g=g_{0}$ preserving the extended $\operatorname{Lsp}(M, g)$ (including multiplicities), one has

$$
\int_{\lambda} \dot{g} d s=0, \forall \lambda
$$

When the cohomology is trivial, one can therefore write $\dot{g}=\Xi(f)$ for some $f$ with the given regularity.

One does not expect the cohomology to be trivial in general settings, but the results might be interesting for the length spectral deformation problem.

\section{PROPERTIES OF ISOSPECTRAL BILLIARDS}

The existence of isospectral pairs proves that the knowledge of the infinite set of eigenenergies does not suffice to uniquely determine the shape of a billiard boundary. A natural question arises: if the set of eigenvalues is not sufficient to distinguish two isospectral billiards, then which quantity, which quantities would suffice to uniquely specify which is which?

In this section we review various elements that have been brought forward to discriminate between two isospectral billiards. 


\section{A. Weyl expansion}

The problem of calculating the eigenvalue distribution for a given domain $B$ (sometimes called Weyl's problem) is dealt with starting from the density of energy levels

$$
d(E)=\sum_{n} \delta\left(E-E_{n}\right)
$$

where $\delta$ is Dirac delta function and the sum runs over all eigenvalues of the system. The counting function is its integrated version:

$$
N(E)=\sum_{n} \Theta\left(E-E_{n}\right)
$$

where $\Theta$ is the Heaviside step function. Statistical functions of the energy can be studied by proper smoothing of the delta functions in (65). The mean of a function $f$ of the energy is defined by its convolution with a test-function $\xi$ :

$$
\bar{f}(E)=\int_{-\infty}^{\infty} f(e) \xi(E-e) d e
$$

The test-function $\xi$ is taken to be centered at 0 , normalized to 1 and have an important weight only around the origin, with a width $\Delta E$ large compared to the mean level spacing but small compared to $E$.

We want to study the mean behaviour of $N(E)$. Suppose the Hamiltonian of an $N$ dimensional system is of the form

$$
H(q, p)=p^{2} / 2 m+V(q)
$$

The "Thomas-Fermi approximation" consists of making the assumption that each quantum state is associated to a volume $(2 \pi \hbar)^{N}$ in phase space. The mean value of $N(E)$ is given by

$$
\begin{aligned}
\bar{N}(E) & \simeq \int \frac{d^{N} p d^{N} q}{(2 \pi \hbar)^{N}} \Theta(E-H(q, p)) \\
& \simeq \frac{1}{\Gamma(N / 2+1)}\left(\frac{m}{2 \pi \hbar^{2}}\right)^{N / 2} \int_{V(q)<E}[E-V(q)]^{N / 2} d q
\end{aligned}
$$


after having integrated over $p$. In the case where we want to describe the movement in an $n$-dimensional box we get

$$
\bar{N}(E) \simeq \frac{\mathcal{V}}{\Gamma(N / 2+1)}\left(\frac{m}{2 \pi \hbar^{2}}\right)^{N / 2} E^{N / 2}
$$

which is the first term in a series expansion of $\bar{N}(E)$, called Weyl expansion, and $\mathcal{V}$ is the volume of the box. For two-dimensional billiards and under our conventions on units, this first term of Weyl expansion reads (for both Dirichlet and Neumann boundary conditions)

$$
\bar{N}(E) \simeq \frac{\mathcal{A}}{4 \pi} E
$$

where $\mathcal{A}$ is the area of the billiard. For the mean density of states, the Thomas-Fermi approximation gives

$$
\bar{d}(E)=\int \frac{d^{N} p d^{N} q}{(2 \pi \hbar)^{N}} \delta(E-H(q, p)) .
$$

For two-dimensional billiards, the first term in the Weyl expansion thus gives a mean density of states equal to

$$
\bar{d}=\frac{\mathcal{A}}{4 \pi} .
$$

The following terms in Weyl expansion are obtained by asymptotic expansion of the Laplace transform of the density of states, defined by

$$
Z(t)=\sum_{n} e^{-E_{n} t}
$$

For polygonal billiards, it was shown in (Bailey and Brownell, 1962) (see also (Kač, 1966) for Dirichlet boundary conditions and in (Pleijel, 1953-1954; Sleeman, 1982) for Neumann boundary conditions) that this expansion for $t \rightarrow 0$ reads

$$
Z(t)=\frac{\mathcal{A}}{4 \pi t} \mp \frac{\mathcal{L}}{8 \sqrt{\pi t}}+\beta+O(\exp (- \text { const } / t))
$$

where $\mathcal{L}$ is the perimeter of the billiard and $\beta$ is a constant depending on the connectivity of the domain and on the corners of the boundary. The sign before $\mathcal{L}$ is $(-)$ for Dirichlet boundary conditions and $(+)$ for Neumann boundary conditions. Brownell (Brownell, 1957) showed that for multiply connected domains with smooth boundary and $p$ smooth 
holes $\beta=(p-1) / 6$. Asymptotic expansion of $Z(t)$ including contributions of corners is given in (Stewardson and Waechter, 1971) (following a method described in (McKean and Singer, 1967)) for boundaries with smooth arcs of length $\gamma_{i}$ and corners of angle $0<\alpha_{j} \leq 2 \pi$. For Dirichlet boundary conditions it reads

$$
Z(t) \simeq \frac{\mathcal{A}}{4 \pi t}-\frac{\mathcal{L}}{8 \sqrt{\pi t}}+\sum_{j} \frac{1}{24}\left(\frac{\pi}{\alpha_{j}}-\frac{\alpha_{j}}{\pi}\right)+\sum_{i} \frac{1}{2 \pi} \int_{\gamma_{i}} \kappa(l) d l
$$

where $\kappa(l)$ is the curvature measured along the arc. (The inward-pointing cusp $\alpha=2 \pi$ is correctly accounted for, while the outward-pointing cusp should be treated separately, see (Baltes and Hilf, 1976).) This allows to obtain an expansion of $\bar{N}(E)$ controlled by logarithmic Gaussian error estimates (see (Baltes and Hilf, 1976) for detailed definitions):

$$
\bar{N}(E) \simeq \frac{1}{4 \pi} \mathcal{A} E \mp \frac{1}{4 \pi} \mathcal{L} \sqrt{E}+\mathcal{K}
$$

where $\mathcal{L}$ is the perimeter of the billiard and $\mathcal{K}$ a constant depending on the geometry of the boundary. The sign before $\mathcal{L}$ is $(-)$ for Dirichlet boundary conditions and $(+)$ for Neumann boundary conditions. For polygonal billiards with angles $\theta_{i}$, the constant is deduced from (76) to be

$$
\mathcal{K}=\frac{1}{24} \sum_{\text {angle } i}\left(\frac{\theta_{i}}{\pi}-\frac{\pi}{\theta_{i}}\right)
$$

As one can see, the statistical behaviour is determined by certain characteristic quantities of the boundary of the billiard, like area, perimeter, or angles. Conversely, if the spectrum is known, it determines the area and the perimeter of the billiard, and it gives the constant $\mathcal{K}$. In particular, isospectral billiards must have the same area and the same perimeter. In the case of polygonal billiards, the fact that $\mathcal{K}$ must be the same as well entails relations between the angles of the billiards.

\section{B. Periodic orbits}

\section{Green function}

We define the propagator of the system as the conditional probability amplitude $K\left(q_{f}, t_{f} ; q_{i}, t_{i}\right)$ for the particle to be at point $q_{f}$ at time $t_{f}$, knowing that it was at point 
$q_{i}$ at time $t_{i}$. The propagator is the only solution of the Schrödinger equation that satisfies condition

$$
\lim _{t_{f} \rightarrow t_{i}} K\left(q_{f}, t_{f} ; q_{i}, t_{i}\right)=\delta\left(q_{f}-q_{i}\right) .
$$

One can then show (see (Gutzwiller, 1991) and references therein) that the propagator can be written as a Feynman integral

$$
K\left(q_{f}, t_{f} ; q_{i}, t_{i}\right)=\int \mathcal{D} q(t) e^{\frac{i}{\hbar} \int d t \mathcal{L}(\dot{q}, q, t)}
$$

where the sum runs over all possible trajectories going from $\left(q_{i}, t_{i}\right)$ to $\left(q_{f}, t_{f}\right)$. The notation (80) has to be understood as the limit as $n$ goes to infinity of a discrete sum over all $n$ step paths going from $\left(q_{i}, t_{i}\right)$ to $\left(q_{f}, t_{f}\right)$ : the sum (80) runs over all continuous, but not necessarily derivable, paths. One immediately sees that the classical limit of quantum mechanics corresponds to letting the constant $\hbar$ go to 0 : the main contributions to the probability $K$ then correspond to stationary points of the action $\int d t \mathcal{L}(\dot{q}, q, t)$ (see (Berry, 1991)).

The advanced Green function is the Fourier transform of the propagator. It is defined by

$$
G\left(q_{f}, q_{i} ; E\right)=\frac{1}{i \hbar} \int_{0}^{\infty} d t K\left(q_{f}, t ; q_{i}, 0\right) e^{i E t / \hbar}
$$

It is a solution of the equation

$$
(-H+E) G\left(q_{f}, q_{i} ; E\right)=\delta\left(q_{f}-q_{i}\right)
$$

The action along a trajectory can be defined as the integral of the momentum

$$
S\left(q_{f}, q_{i} ; E\right)=\int_{q_{i}}^{q_{f}} p d q
$$

and the Green function as

$$
G\left(q_{f}, q_{i} ; E\right)=\frac{1}{i \hbar} \int \mathcal{D} q(t) e^{\frac{i}{\hbar} S\left(q_{f}, q_{i} ; E\right)}
$$

The Green function is thus a sum over all continuous paths from $q_{i}$ to $q_{f}$. In many cases equation (82) allows to calculate the Green function. In the case of free motion in Eu- 
clidean space, the Hamiltonian reduces to the Laplacian (up to a sign), and the Green function is solution of

$$
\left(\Delta_{q_{f}}+E\right) G\left(q_{f}, q_{i} ; E\right)=\delta\left(q_{f}-q_{i}\right)
$$

where the $q_{f}$ index recalls that the derivatives of the Laplacian are applied on variable $q_{f}$. In two dimensions, Green's function is

$$
G\left(q_{f}, q_{i} ; E\right)=\frac{1}{4 i} H_{0}^{(1)}\left(k\left|q_{f}-q_{i}\right|\right)
$$

with $k=\sqrt{E} ; H_{0}^{(1)}$ is a Hankel function of the first kind. In dimension 3 we get

$$
G\left(q_{f}, q_{i} ; E\right)=\frac{e^{i k\left|q_{f}-q_{i}\right|}}{2 i k}
$$

\section{Semiclassical Green function}

Semiclassical methods are based on the fact that the classical limit of quantum mechanics is obtained for $\hbar \rightarrow 0$ in the path integral expressing the propagator. The expansion of this integral in powers of $\hbar$ allows to calculate the sequence of quantum corrections to classical theory. The semiclassical approximation only keeps in this expansion the lowest-order term in $\hbar$. Corrections to this approximation correspond to taking into account higherorder terms. This semiclassical approximation is therefore valid only if the following term is negligeable, that is if $S / \hbar \gg 1$.

The expression (84) for Green's function $G\left(q_{f}, q_{i} ; E\right)$ is a sum over all continuous paths joining $q_{i}$ to $q_{f}$ at energy $E$. The semiclassical approximation consists in keeping only the lowest-order term in the $\hbar$ expansion. This term is given by stationary phase approximation. The only paths contributing to the integral (84) are paths for which the action $S$ reaches a stationary value, that is, paths that correspond to classical trajectories. The semiclassical Green function can thus be expressed as a sum, over all classical trajectories, of exponentials whose phase is, up to a $\pi / 2$ multiple, the classical action integrated along the trajectory. The same approximation can be obtained for the Feynman propagator (80). Integration of (80) (or, more precisely, of its discretized version) by stationary phase approximation is due to Van Vleck. Gutzwiller (Gutzwiller, 1991) obtained an expression for the semiclassical Green function. Stationary points correspond to classical trajectories. In- 
tegrating with respect to time and then choosing a coordinate system $\left(q_{\|}, q_{\perp}\right)$ such that $q_{\|}$is the coordinate along the trajectory and $q_{\perp}$ the coordinates perpendicular to the trajectory, one obtains the semiclassical Green function as a sum over all classical trajectories:

$$
\begin{aligned}
G^{\text {s.c. }}\left(q_{f}, q_{i} ; E\right)=\sum_{\mathrm{cl}} & \frac{2 \pi}{(2 i \pi \hbar)^{(N+1) / 2}}\left[\frac{1}{\dot{q}_{i_{\|}} \dot{q}_{f_{\|}}} \operatorname{det}\left(-\frac{\partial^{2} S}{\partial q_{f_{\perp}} \partial q_{i_{\perp}}}\right)\right]^{1 / 2} \\
& \times \exp \left(\frac{i}{\hbar} S\left(q_{f}, q_{i} ; E\right)-i \mu \frac{\pi}{2}\right) .
\end{aligned}
$$

$N$ is the space dimension. To obtain (88), the action (83) has been expanded around classical trajectories of energy $E$ going from $q_{i}$ to $q_{f}$. The second order of the expansion is a quadratic form in the position $q_{f}$. We call a conjugate point a point (of the trajectory around which the action is expanded) where one eigenvalue of this quadratic form becomes negative (close to the starting point $q_{i}$ the quadratic form is positive definite). In Equation (88), the index $\mu$ depends on the classical trajectory considered: it counts the number of conjugate points along the trajectory. Physically, a conjugate point corresponds to a point of the trajectory where there is an "inversion" of a pencil of nearby trajectories, either as a caustic or as a focal point. It is the case in particular in two dimensions for hard wall reflexions. Each reflexion yields a contribution $\mu=2$ for Dirichlet boundary conditions and 0 for Neumann or periodic boundary conditions.

Since we will mainly be interested in the trace formula in two-dimensional polygonal billiards, we only state the result in this case. The classical action along a periodic orbit of length $l_{p}$ is given by

$$
S_{p}=\oint p d q=k l_{p}
$$

To each trajectory is associated its Maslov index $\mu_{p}$. The semiclassical Green function reads

$$
G^{\text {s.c. }}(q, q ; E)=\sum_{\vec{l}_{p}} \frac{e^{i k l_{p}-i \mu_{p} \frac{\pi}{2}-3 i \frac{\pi}{4}}}{\sqrt{8 \pi k l_{p}}}
$$

with $k=\sqrt{E}$. 


\section{Density of states}

The Green function of a quantum system is defined by (81). It will be more useful to express Green's function as a sum over eigenvalues and eigenfunctions of the Hamiltonian, according to (82). It can be verified that formally

$$
G\left(q_{f}, q_{i} ; E\right)=\sum_{n} \frac{\bar{\Psi}_{n}\left(q_{i}\right) \Psi_{n}\left(q_{f}\right)}{E-E_{n}}
$$

where $\bar{\Psi}$ denotes the complex conjugate of $\Psi$, is indeed a solution of (82). In order to give a mathematically correct meaning to this expression, we use the advanced Green function

$$
G_{+}\left(q_{f}, q_{i} ; E\right)=G\left(q_{f}, q_{i} ; E+i \epsilon\right)
$$

The words "Green function" will always implicitly refer to the limit of the advanced Green function for $\epsilon \rightarrow 0$. We use the fact that for $\epsilon \rightarrow 0$,

$$
\lim _{\epsilon \rightarrow 0} \frac{1}{x+i \epsilon}=\operatorname{pv} \frac{1}{x}-i \pi \delta(x)
$$

(pv denotes the principal value and $\delta$ Dirac delta function), and that, since $H$ is Hermitian, its eigenvectors verify $\int \bar{\Psi}_{m} \Psi_{n}=\delta_{m n}$. The density of energy levels (65) can thus be related to the Green function by

$$
d(E)=-\frac{1}{\pi} \int \mathcal{I} m G(q, q ; E) d q
$$

The Green function $G\left(q^{\prime}, q ; E\right)$ diverges for $q^{\prime} \rightarrow q$ but not its imaginary part. Expression Im $G(q, q ; E)$ has to be understood as the imaginary part of $G\left(q^{\prime}, q\right)$ taken at the limit $q^{\prime} \rightarrow q$. Thanks to this relation, the density of states can be expressed as the trace of Green's function. Equation (94) is the starting point of trace formulae. Note that if the density of states (65) is regularized as a sum of Lorentzians

$$
d_{\epsilon}(E)=\frac{\epsilon}{\pi} \sum_{n} \frac{1}{\left(E-E_{n}\right)^{2}+\epsilon^{2}},
$$


one gets

$$
d_{\epsilon}(E)=-\frac{1}{\pi} \int \mathcal{I} m G(q, q ; E+i \epsilon) d q
$$

Equation (94) must therefore be understood as the limit, as $\epsilon \rightarrow 0$, of each member of Equation (96). However the density of states is usually calculated from the Green function by first evaluating the integral for $q=q^{\prime}$ (the "trace" of the Green function), then taking the imaginary part. This can be made rigorous, by multiplying the Green function by some factor making the integral convergent in the limit $q=q^{\prime}$ (Balian and Bloch, 1974).

The density of states in the semiclassical approximation is then the sum of a "smooth part" and an oscillating term which is a superposition of plane waves:

$$
d(E)=\bar{d}(E)+d^{\mathrm{osc}}(E)
$$

where $\bar{d}$ is the Thomas-Fermi term (73), and

$$
d^{\mathrm{osc}}(E) \simeq \frac{i}{(2 i \pi \hbar)^{(N+1) / 2}} \sum_{\mathrm{ppo}, \mathrm{n}} \frac{T_{p}}{\left|\operatorname{det}\left(M_{p}^{n}-\mathbb{I}\right)\right|^{1 / 2}} e^{i n\left(\frac{k l_{p}}{\hbar}-\nu_{p} \frac{\pi}{2}\right)}+\text { c.c. }
$$

the index $\nu_{p}$ now taking into account additional phases due to integration. The identity matrix is denoted by $\mathbb{I}, M_{p}$ is the monodromy matrix associated to the orbit $p$ and c.c. denotes the conjugated complex. Gutzwiller trace formula (98) is a sum over all primitive periodic orbits and all repetition numbers $n$. It is a formal sum: convergence issues will be left aside in this review.

In the case of integrable and pseudo-integrable systems, periodic orbits are no longer isolated but appear within families, of parallel trajectories having the same length ("cylinders of periodic orbits"). The Gutzwiller trace formula does not apply any more. In (Berry and Tabor, 1976), Berry and Tabor derived a trace formula for multidimensional integrable systems. In the case of a two-dimensional polygonal billiard, the trace formula becomes

$$
d(E) \simeq \bar{d}+\sum_{\mathrm{pp}} \frac{\mathcal{A}_{p}}{2 \pi} \sum_{n=1}^{\infty} \frac{e^{i k n l_{p p}-3 i \pi / 4-i n \nu_{p p} \pi / 2}}{\sqrt{8 \pi k n l_{p p}}}+\text { c.c. }
$$

where $\mathcal{A}_{p}$ is the area occupied by the cylinder of periodic orbits labeled by $p$.

It can be proved fairly easily, using transplantation, that two isospectral domains have the 
same length spectrum (i.e. both domains have periodic orbits of the same length) (Okada and Shudo, 2001a). It is possible to encode any trajectory drawn on the billiard (provided it does not pass through vertices) by symbolic dynamics. A trajectory is labeled by the sequence of edges of the base tile that it crosses on its way.

Consider again the example of Fig. 2. Fig. 8 shows two pencils of periodic orbits on each billiard. One can check that these two pencils appear with the same length and the same width in both billiards.
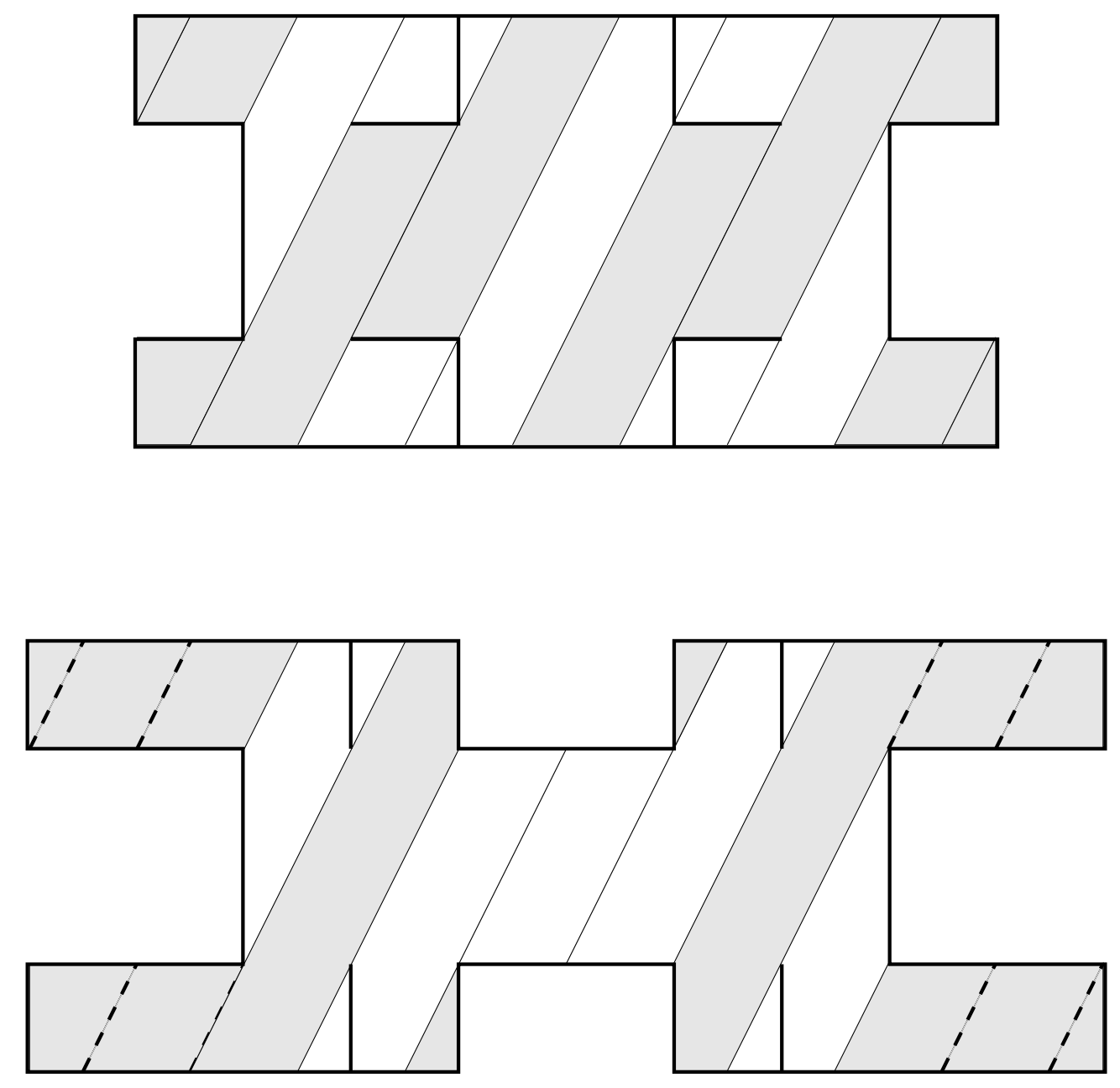

Figure 8 Periodic and diffractive orbits in the unfolded pair $7_{3}$.

Conversely, S. Fulling and P. Kuchment have proven in (Fulling and Kuchment, 2005) that "Coincidence of length spectra does not imply isospectrality", giving the explicit example of a so-called Penrose-Lifshits mushroom (see section VIII.B). 


\section{Diffractive orbits}

When the system contains scattering points, the semiclassical trace formula (99) has to be modified. The semiclassical density of states includes a term associated to diffractive trajectories, that is classical trajectories going from one scattering point to another (Keller, 1962; Vattay et al., 1994).

In the case of polygonal billiards, Hannay and Thain (Hannay and Thain, 2003) have been able to derive an exact expansion for the Green function, as a sum over all scattering trajectories. It reads

$$
\begin{aligned}
G(a, b) & =\sum_{n=0}^{\infty} \frac{1}{(2 \pi)^{n}} \sum_{\substack{n \text { vertex } \\
\text { paths }}} \frac{1}{2 i} \int_{-\infty}^{\infty} d s_{1} d s_{2} \ldots d s_{n} H_{0}^{(1)}\left[k R\left(s_{1}, s_{2}, \ldots, s_{n}\right)\right] \\
& \times \prod_{k=1}^{n} \frac{2 \pi}{\left(\gamma_{k} M_{k}+\theta_{k}+i s_{k}\right)^{2}-\pi^{2}}
\end{aligned}
$$

where

$$
\begin{array}{r}
R^{2}\left(s_{1}, s_{2}, \ldots, s_{n}\right)=\left(r_{0}+r_{1} e^{s_{1}}+r_{2} e^{s_{1}+s_{2}}+\cdots+r_{n} e^{s_{1}+s_{2}+\cdots+s_{n}}\right) \\
\times\left(r_{0}+r_{1} e^{-s_{1}}+r_{2} e^{-s_{1}-s_{2}}+\cdots+r_{n} e^{-s_{1}-s_{2}-\cdots-s_{n}}\right) .
\end{array}
$$

The Green function appears as a sum over paths made of $n$ straight lines going from a diffracting corner to another. The diffraction angles are $M_{k} \gamma_{k}+\theta_{k}, 1 \leq k \leq n$, with $\gamma_{k}$ the measure of the angle at the singularity and $M_{k}$ the number of times the path winds around the singularity (thus, $0 \leq \theta_{k}<\gamma_{k}$ ).

In (Giraud, 2004) it was shown that isospectral domains can be distinguished by the fact that in general the lengths of their diffractive orbits differs. This can be illustrated e. g. in the case of the billiard with rectangular base tile unfolded to a translation surface (Fig. 8). If the sides of the base tiles are incommensurate, then for a given diffractive orbit of one billiard a diffractive orbit of same length in the other billiard has to be in the same direction. For the dashed diffractive orbit drawn in the second billiard of Fig. 8, orbits starting from a diffractive corner of the first billiard in the same direction never reach another diffractive corner.

The connection between the energy spectrum and the length spectrum through the trace 
formula indicates however that these discrepancies between diffractive orbits must be compensated in a certain way. This can be understood by analyzing the formula of Hannay and Thain (100). In fact each contribution to the Green function in (100) has to be understood as an infinite sum over all windings around vertices (i.e. the four corners and the two points at the millde of the horizontal sides of each of the seven rectangular tiles in Fig. 7). If there is a scatterer (as is the case for instance at the bottom right corner of
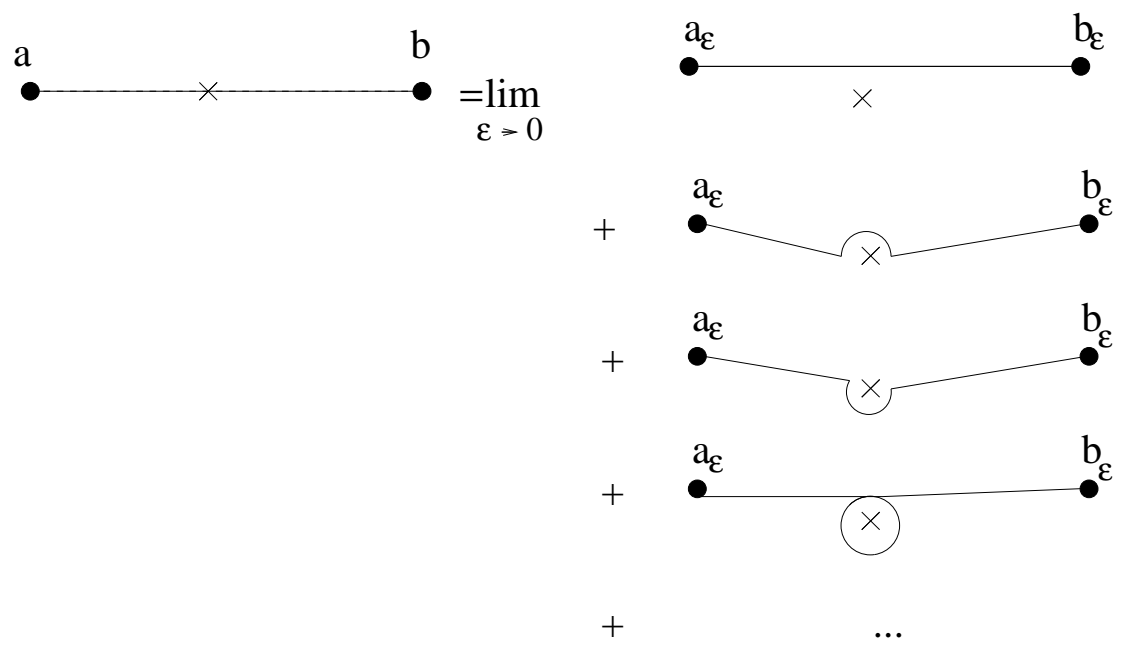

Figure 9 A contribution to the Green function in case of forward diffraction. If the orbit goes through a vertex the term in (100) should be interpreted as the limit for $\epsilon \rightarrow 0$ of an infinite number of trajectories. If there is no vertex only the straight path contribution remains, the other (winding) terms add up to zero.

tile 7 in the second billiard of Fig. 7) then there is a non-zero contribution, while if there is no scatterer (e.g. at the bottom left corner of tile 7 in the second billiard) the series of diffractive terms adds up to zero:

$$
\sum_{M_{k}=-\infty}^{\infty} \frac{2 \pi}{\left(2 \pi M_{k}+\pi+i s_{k}\right)^{2}-\pi^{2}}=0 .
$$

As a consequence, a diffractive contribution to the Green function, going from a point $a$ to a point $b$ through possibly several vertices, has to be understood as a sum of trajectories winding around scattering or non-scattering vertices (see Fig. 10). Now each of these new "ficticious" trajectories avoid vertices (since they wind around), and to each of them in one billiard it is always possible to find a partner in the other billiard. 


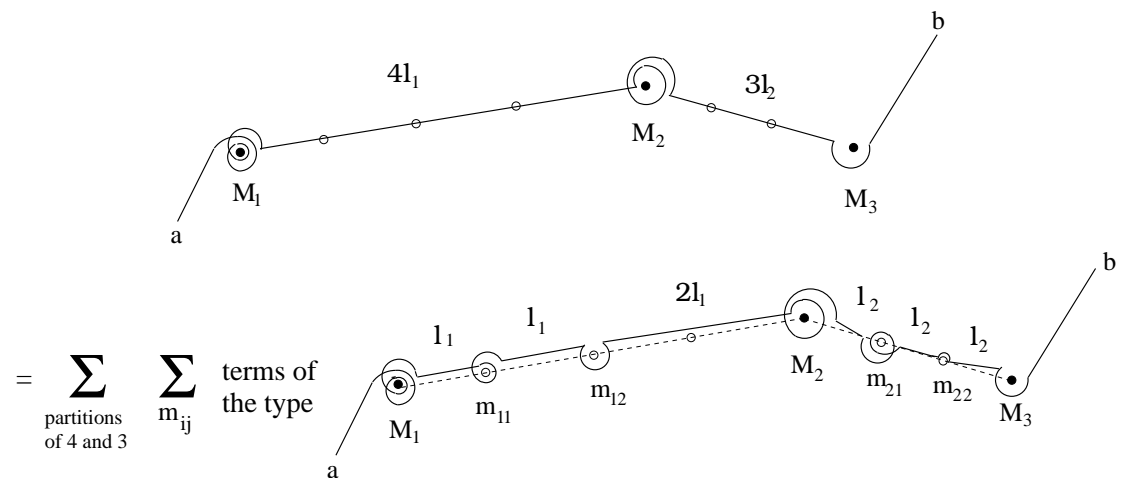

Figure 10 A contribution to the Green function in case of forward diffraction. The filled circles are scattering vertices, the empty ones are non-scattering vertices.

\section{Green function}

This compensation is made clear through a relation between the Green functions of the two domains (Giraud, 2004). Recall that the way the building blocks are glued together (or, equivalently, the colouring of the associated graph) can be described by matrices $M^{(\mu)}, N^{(\mu)}, 1 \leq \mu \leq 3$, as introduced in section I. The transplantation between the two billiards can be described by some matrix $T$. It turns out that these matrices verify the property

$$
\sum_{i^{\prime}, j^{\prime}} T_{i, i^{\prime}} T_{j, j^{\prime}} M_{i^{\prime}, j^{\prime}}^{(A)}=1+2 M_{i, j}^{(B)},
$$

which can be proved using the commutation relation (9) of the $M^{(\mu)}$ (that will be studied in more detail in section VII) and the fact that $\left(T^{2}\right)_{i j}=1+2 \delta_{i j}$. To each of the "ficticious" trajectories described above one can associate a "word" $\left(a_{1}, a_{2}, \ldots, a_{n}\right)$ describing the edges crossed by the trajectory, and a matrix $M=\prod M^{\left(a_{i}\right)}$. This trajectory exists between tiles $i$ and $j$ if and only if $M_{i j}=1$. Thus, in the expansion (100) of the Green function between a point in tile $i$ and a point in tile $j$ in the first (second) billiard, each trajectory appears with a weight $M_{i j}^{A}\left(M_{i j}^{B}\right)$. But according to Eq. (103) we have

$$
M_{i j}^{B}=\frac{1}{2} \sum_{i^{\prime}, j^{\prime}} T_{i, i^{\prime}} T_{j, j^{\prime}} M_{i^{\prime} j^{\prime}}^{A}-\frac{1}{2} .
$$


Therefore from Eq. (100) and identity (104) one can infer a relation between Green functions, namely

$$
G^{(B)}(a, i ; b, j)=\frac{1}{2} \sum_{i^{\prime}, j^{\prime}} T_{i, i^{\prime}} T_{j, j^{\prime}} G^{(A)}\left(a, i^{\prime} ; b, j^{\prime}\right)-\frac{1}{2} G^{(t)}(a ; b),
$$

where $G^{(t)}(a ; b)$ is the Green function on the base tile.

\section{E. Scattering poles of the exterior Neumann problem}

\section{Fredholm theory}

In section V.C we considered the particular case of polygonal isospectral billiards, for which the expansion of (Hannay and Thain, 2003) exists. In a more general setting, it is also possible to express the Green function with Dirichlet boundary conditions as an infinite sum taking into account all possible reflexions on obstacles. Balian and Bloch (Balian and Bloch, 1974) gave a general method, called "multiple reflexion expansion", which gives the Green function in terms of the free Green function $G_{0}$. Applied to a twodimensional billiard this gives

$$
\begin{aligned}
G\left(q, q^{\prime} ; E\right) & =G_{0}\left(q, q^{\prime} ; E\right)-2 \int_{\partial B} d s G_{0}(q, s ; E) \partial_{s} G_{0}\left(s, q^{\prime} ; E\right) \\
& +(-2)^{2} \int_{\partial B} d s d s^{\prime} \partial_{s} G_{0}(q, s ; E) \partial_{s^{\prime}} G_{0}\left(s, s^{\prime} ; E\right) G_{0}\left(s^{\prime}, q^{\prime} ; E\right)+\cdots
\end{aligned}
$$

where $s, s^{\prime}$ are points along the boundary, and $\partial_{x}$ denotes here the derivative along an outward vector normal to the boundary at point $x$. The term $G_{0}\left(q, q^{\prime} ; E\right)$ on the right hand of (106) corresponds to direct (free) propagation from $q$ to $q^{\prime}$, the first integral corresponds to trajectories from $q$ to $q^{\prime}$ with one reflexion on the boundary at point $s$, etc... We introduce the kernel $K_{E}\left(q, q^{\prime}\right)=-2 \partial_{q^{\prime}} G_{0}\left(q, q^{\prime} ; E\right)$, which a continuous infinitedimensional operator defined on $\partial B \times \partial B$. One can express (106) as

$$
\begin{aligned}
G\left(q, q^{\prime} ; E\right) & =G_{0}\left(q, q^{\prime} ; E\right) \\
& -2 \sum_{n=0}^{\infty} \int_{\partial B} d s d s^{\prime} G_{0}(q, s ; E) K_{E}^{n}\left(s, s^{\prime}\right) \partial_{s^{\prime}} G_{0}\left(s^{\prime}, q^{\prime} ; E\right) .
\end{aligned}
$$


Performing formally the sum over $n$ yields the infinite-dimensional operator $\left(\mathbb{I}-K_{E}\right)^{-1}$. Fredholm theory (Smithies, 1962) shows that, for sufficiently "nice" billiards, the operator $\left(\mathbb{I}-K_{E}\right)^{-1}$ is well-defined and can be expressed as

$$
\left(\mathbb{I}-K_{E}\right)^{-1}=\frac{N_{E}}{D(E)},
$$

where $D(E)$ is the Fredholm determinant $\operatorname{det}\left(\mathbb{I}-K_{E}\right), N_{E}$ is an infinite-dimensional operator defined on $\partial B \times \partial B$, and $\mathbb{I}$ is the identity operator. The Fredholm determinant admits an expansion

$$
D(E)=\sum_{0}^{\infty} D_{n}(E)
$$

with

$$
D_{n}(E)=\frac{(-1)^{n}}{n !} \int_{\partial B} d q_{1} \ldots \int_{\partial B} d q_{n} K\left(\begin{array}{l}
q_{1}, q_{2}, \ldots, q_{n} \\
q_{1}, q_{2}, \ldots, q_{n}
\end{array}\right)
$$

and $D_{0}(E)=1$. We have introduced the determinant

$$
K\left(\begin{array}{c}
q_{1}, q_{2}, \ldots, q_{n} \\
q_{1}^{\prime}, q_{2}^{\prime}, \ldots, q_{n}^{\prime}
\end{array}\right)=\left|\begin{array}{cccc}
K_{E}\left(q_{1}, q_{1}^{\prime}\right) & K_{E}\left(q_{1}, q_{2}^{\prime}\right) & \ldots & K_{E}\left(q_{1}, q_{n}^{\prime}\right) \\
K_{E}\left(q_{2}, q_{1}^{\prime}\right) & K_{E}\left(q_{2}, q_{2}^{\prime}\right) & \ldots & K_{E}\left(q_{2}, q_{n}^{\prime}\right) \\
\ldots & \ldots & \ldots & \ldots \\
K_{E}\left(q_{n}, q_{1}^{\prime}\right) & K_{E}\left(q_{n}, q_{2}^{\prime}\right) & \ldots & K_{E}\left(q_{n}, q_{n}^{\prime}\right)
\end{array}\right|
$$

The operator $N_{E}$ is defined on $\partial B \times \partial B$ by its expansion $N_{E}=\sum_{0}^{\infty} N_{n}$ with

$$
N_{n}=\sum_{k=0}^{n} D_{k}(E) K_{E}^{n-k}
$$

The Fredholm determinant $D(E)$ has the property that it has zeros at eigenvalues of the system (Georgeot and Prange, 1995). It has been shown in (Tasaki et al., 1997) that for billiards with $C^{2}$ boundary, $D(E)$ can be decomposed as an interior and an exterior contribution, namely $D(E)=D(0) d_{\text {int }}(E) d_{\text {ext }}(E)$. The exterior contribution $d_{\text {ext }}(E)$ is related to the scattering of a wave on an obstacle having the shape of the billiard with Neumann boundary conditions, i.e. the zeros of its analytic continuation are resonances of the exte- 
rior scattering problem. The interior contribution reads

$$
d_{\text {int }}(E)=e^{i \frac{\mathcal{A} E}{4}}\left(\frac{\mathcal{L}^{2} E}{4}\right)^{-\frac{\mathcal{A} E}{4 \pi}} e^{-\frac{\mathcal{A} \gamma E}{2 \pi}} \prod_{n=1}^{\infty}\left(1-\frac{E}{E_{n}}\right) e^{E / E_{n}}
$$

where $\mathcal{A}$ and $\mathcal{L}$ are respectively the area and the perimeter of the billiard, and $\gamma$ a constant depending on the geometry of the billiard. The zeros of $d_{\text {int }}(E)$ are thus the eigenenergies of the interior Dirichlet problem.

Obviously, isospectral billiards share the same interior part $d_{\text {int }}(E)$. But calculating the Fredholm determinant requires the knowledge of the whole shape of the billiard. In particular solutions of the exterior Neumann scattering problem may differ between two isospectral billiards. Therefore isospectral pairs might be distinguished by measuring the sound scattered by them (Tasaki et al., 1997).

To check this property, numerical investigations have been performed in (Okada et al., 2005a). In fact, Fredholm theory applies only for billiards with a smooth boundary, which is not the case for any of the known examples (see B). For billiards with a piecewise smooth boundary it is however possible to approximate $D(E)$ by a discretized version $D^{m}(E)$, depending on the number $m$ of points taken on the boundary of the billiard, and which converges to $D(E)$ for large $m$. This convergence fails for boundaries with corners: all $D^{m}(E)$ tend to 0 . Nevertheless, for domains with corners (Okada et al., 2005b) showed that it is possible to define a regularized version of $D^{m}(E)$ that converges to $D(E) / D(0)=d_{\text {int }}(E) d_{\text {ext }}(E)$. Using this regularized version, zeros of the regularized Fredholm determinant were computed numerically for various pairs of isospectral billiards in (Okada et al., 2005a). It was observed that zeros close to the real axis coincide, as they should since they are eigenvalues of the interior problem. On the other hand complex zeros (remote from the real axis), which correspond to resonances of the exterior Neumann problem, are shown to differ. To quantify this discrepancy between resonances of the two billiards, the resonance counting number

$$
N_{\delta}(r)=\left\{z \in \mathbb{C} ;|z|<r,-\frac{\pi}{2}<\arg (z)<-\delta\right\}
$$

was studied in (Okada et al., 2005a). The best fit $N_{\delta}(r)=C_{\delta, R} r^{2}$, computed over the range $r \in[0, R]$, yields noticeably different values of $C_{\delta, R}$ for each billiard. This clearly proves 
that isospectral pairs can indeed be distinguished by resonances of scattering waves.

\section{F. Eigenfunctions}

\section{Triangular states}

Although in general analytical solutions to the Helmholtz equation $(\Delta+E) \Psi=0$ with Dirichlet boundary conditions are not known, it is possible to construct particular solutions of this equation as eigenfunctions coinciding with those of the elementary sub-domains of the billiards. Let us take the example of the two billiards in Fig. 11, where each billiard in Fig. 11 is made of 7 triangular (half-square) tiles.

Eigenfunctions for a $d \times d$-square with Dirichlet boundary conditions are of the form

$$
s_{m, n}(x, y)=\frac{4}{d^{2}} \sin \left(\frac{m \pi x}{d}\right) \sin \left(\frac{n \pi y}{d}\right)
$$

with eigenvalues $E_{m, n}=\pi^{2}\left(m^{2}+n^{2}\right) / d^{2}, m, n \geq 1$. Eigenfunctions for the elementary triangles with Dirichlet boundary conditions are obtained from (115) by antisymmetrization with respect to the diagonal:

$$
t_{m, n}(x, y)=\frac{4}{d^{2}}\left[\sin \left(\frac{m \pi x}{d}\right) \sin \left(\frac{n \pi y}{d}\right)-\sin \left(\frac{m \pi y}{d}\right) \sin \left(\frac{n \pi x}{d}\right)\right] .
$$

The corresponding eigenenergies are given by $\pi^{2}\left(m^{2}+n^{2}\right) / d^{2}, m>n$. The functions $t_{m, n}$ turn out to be also elementary solutions of the Helmholtz equation for both isospectral billiards of Fig. 11. Indeed $t_{m, n}$ vanishes on all lines $x=k d, y=k d, y=x+2 k d$ and $y=-x+2 k d, k \in \mathbb{Z}$, which are precisely the lines on which the boundary of both billiards lie (in the convention of Fig. 11). It is therefore possible to construct particular solutions of Helmholtz equation by taking 7 copies of an eigenfunction of the triangle. These particular solutions are called "triangular states". The labels of the lowest-energy triangular states among the eigenvalues $E_{1} \leq E_{2} \leq \ldots$ of the billiards have been calculated in (Gottlieb and McManus, 1998). The results are displayed in Table V.F.1. Each integer pair $(m, n), m>n$, defines a triangular state $t_{m, n}$. Obviously, the fact that an integer can be represented in more than one way as a sum of two squares leads to degeneracies for triangular states. 

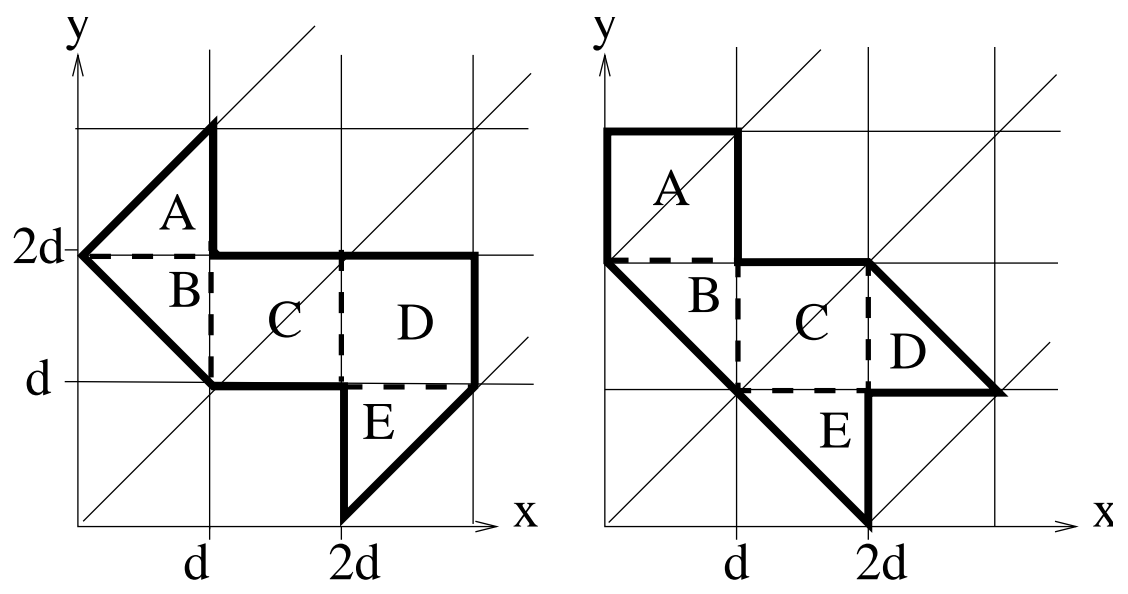

Figure 11 Isospectral billiards divided into smaller regions.

\begin{tabular}{ccc||ccc}
\multicolumn{3}{c||}{$\mathrm{m}$} & $\mathrm{n}$ & \multicolumn{3}{c}{ Eigenvalue } & \multicolumn{3}{r}{ n } & Eigenvalue \\
\hline 1 & 2 & $E_{9}$ & 0 & 1 & $E_{5}$ \\
1 & 3 & $E_{2} 1$ & 1 & 1 & $E_{9}$ \\
2 & 3 & $E_{2} 7$ & 0 & 2 & $E_{1} 5$ \\
1 & 4 & $E_{3} 8$ & 1 & 2 & $E_{2} 0$ \\
2 & 4 & $E_{4} 4$ & 2 & 2 & $E_{2} 9$
\end{tabular}

Table I Rank of the first triangular modes $t_{m, n}$.

Note that for Neumann boundary conditions, it can be easily checked that the functions

$$
u_{m, n}(x, y)=\frac{4}{d^{2}}\left[\cos \left(\frac{m \pi x}{d}\right) \cos \left(\frac{n \pi y}{d}\right)+\cos \left(\frac{m \pi y}{d}\right) \cos \left(\frac{n \pi x}{d}\right)\right]
$$

for $0 \leq m \leq n,(m, n) \neq(0,0)$, have a normal derivative that vanishes on all lines $x=k d$, $y=k d, y=x+2 k d$ and $y=-x+2 k d, k \in \mathbb{Z}$. Therefore $u_{m, n}$ are solutions of Helmholtz equations for the billiards of Fig. 11 with Neumann boundary conditions. Their label among the eigenstates of the billiards is given in Table V.F.1 (Gottlieb and McManus, 1998).

\section{Mode-matching method}

The knowledge of these particular triangular states is the starting point for the so-called "mode-matching method". For the sake of definiteness, we consider again the two isospectral pairs on a Cartesian reference frame, following (Wu et al., 1995), as in Fig. 11. The 
mode-matching method consists in dividing the billiards into subdomains for which solutions of the Helmholtz equation are known analytically. Consider as an example the left billiard of Fig. 11. It is made of the 5 elementary domains, 3 triangles $A, B, E$ and 2 squares $C, D$. Let $d$ be the length of the side of the elementary square, and set $a_{n}=n \pi / d$, $b_{n}=\sqrt{E-a_{n}^{2}}$. We define the function $\psi_{n}(x, y)=\sin \left(a_{n} x\right) \sin \left(b_{n} y\right) / \sin \left(b_{n} d\right)$. For each subdomain, analytical solutions are given by (translations of) functions (115) or (116). If given boundary conditions are imposed on the boundaries of these subdomains, as in Fig. 12, solutions can be written explicitely for these elementary subdomains as superpositions of functions obtained from translations or reflexions of $\psi_{n}$. In particular, one can

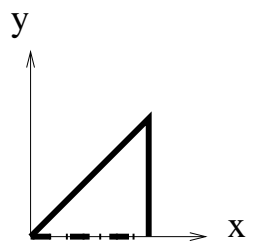

A

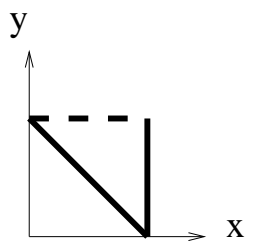

B1

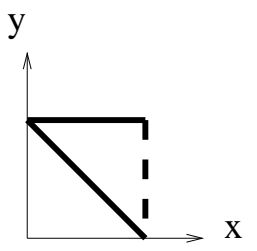

B2

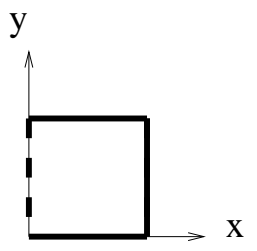

C1

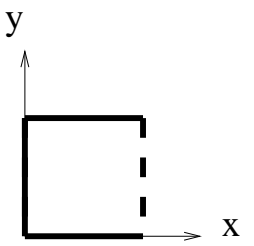

$\mathrm{C2}$

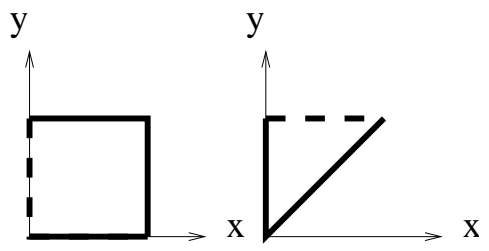

D1

$\mathbf{E}$

Figure 12 Elementary regions building the isospectral pairs.

construct functions taking value 0 on the plain boundary and 1 on the dashed boundary 
for each of the domains depicted in Fig. 12. These functions are given by

$$
\begin{aligned}
\Phi_{n}^{(A)}(x, y) & =\psi_{n}(x, d-y)-\psi_{n}(y, d-x) \\
\Phi_{n}^{(B 1)}(x, y) & =\psi_{n}(x, y)-\psi_{n}(d-y, d-x) \\
\Phi_{n}^{(B 2)}(x, y) & =\psi_{n}(y, x)-\psi_{n}(d-x, d-y) \\
\Phi_{n}^{(C 1)}(x, y) & =\psi_{n}(y, d-x) \\
\Phi_{n}^{(C 2)}(x, y) & =\psi_{n}(y, x) \\
\Phi_{n}^{(D 1)}(x, y) & =\psi_{n}(y, d-x) \\
\Phi_{n}^{(D 2)}(x, y) & =\psi_{n}(x, d-y) \\
\Phi_{n}^{(E)}(x, y) & =\psi_{n}(x, y)-\psi_{n}(y, x) .
\end{aligned}
$$

The mode-matching method consists in looking for a solution $\Psi$ of Helmholtz equation as a superposition of such functions, with amplitudes chosen such that $\Psi$ and its partial derivatives be continuous at each boundary between subdomains. At the boundary between elementary subdomains, the eigenfunction $\Psi$ can be expanded on the functions $\varphi_{n}(x)=\sin \left(a_{n} x\right)$, as

$$
\begin{aligned}
& \Psi_{A B}(x, y)=\sum_{n} A_{n} \varphi_{n}(x) \\
& \Psi_{B C}(x, y)=\sum_{n} B_{n} \varphi_{n}(y-d) \\
& \Psi_{C D}(x, y)=\sum_{n}^{n} C_{n} \varphi_{n}(y-d) \\
& \Psi_{D E}(x, y)=\sum_{n} D_{n} \varphi_{n}(x-2 d),
\end{aligned}
$$

where the sum goes from 1 to some truncation number $N$. The value of the eigenfunction $\Psi$ only depends on the value of the vector $\mathrm{V}=$ 
$\left(A_{1}, \ldots, A_{n}, B_{1}, \ldots, B_{n}, C_{1}, \ldots, C_{n}, D_{1}, \ldots, D_{n}\right)$. Therefore $\Psi$ can be written as

$$
\begin{aligned}
& \Psi_{A}(x, y)=\sum_{n} A_{n} \Phi_{n}^{(A)}(x, y-2 d) \\
& \Psi_{B}(x, y)=\sum_{n} A_{n} \Phi_{n}^{(B 1)}(x, y-d)+\sum_{n} B_{n} \Phi_{n}^{(B 2)}(x, y-d) \\
& \Psi_{C}(x, y)=\sum_{n} B_{n} \Phi_{n}^{(C 1)}(x-d, y-d)+\sum_{n} C_{n} \Phi_{n}^{(C 2)}(x-d, y-d) \\
& \Psi_{D}(x, y)=\sum_{n} C_{n} \Phi_{n}^{(D 1)}(x-2 d, y-d)+\sum_{n} D_{n} \Phi_{n}^{(D 2)}(x-2 d, y-d) \\
& \Psi_{E}(x, y)=\sum_{n} D_{n} \Phi_{n}^{(E)}(x-2 d, y)
\end{aligned}
$$

where $\Psi_{X}$ is the restriction of the function $\Psi$ to the elementary domain $X=A, B, C, D$, or $E$. The function $\Psi$ is indeed an eigenfunction of the billiard if its normal derivatives at the boundaries between domains are continuous. This latter condition can be written as a system of linear equations that can be cast under the form $M \mathbf{V}=0$, where $M$ is a $4 N \times 4 N$-matrix given by

$$
M=\left(\begin{array}{cccc}
U-2 W & P W P-P V / 2 & 0 & 0 \\
P W P-P V / 2 & U-W & -V / 2 & 0 \\
0 & -V / 2 & U & W \\
0 & 0 & W & U-P W P
\end{array}\right)
$$

with $U_{m n}=\left(b_{n} \cot b_{n} d\right) \delta_{m n}, V_{m n}=\left(b_{n} / \sin b_{n} d\right) \delta_{m n}, W_{m n}=a_{m} a_{n} /\left(E-a_{m}^{2}-a_{n}^{2}\right)$ and $P_{m n}=(-1)^{n} \delta_{m n}$. The matrix $M$ depends on $E$ through the $b_{n}$. Eigenvalues of the billiard correspond either to values of $E$ where $\operatorname{det} M=0$ ("real" billiard states) or to $V=0$. In the latter case, the wavefunction vanishes on the boundaries between the domains; it corresponds to triangular states of section V.F.1. Otherwise Eqs. (120) give the general form of eigenfunctions in the billiard.

The mode-matching method provides an alternative proof to isospectrality. Indeed the same method can be applied to the right billiard of Fig. 11. The matrix corresponding to 
$M$ is the $4 N \times 4 N$-matrix $M^{\prime}$ given by

$$
M^{\prime}=\left(\begin{array}{cccc}
U-W & P W P-P V / 2 & 0 & 0 \\
P W P-P V / 2 & U-W & P V / 2 & W \\
0 & P V / 2 & U-W & P W P \\
0 & W & P W P & U-W
\end{array}\right)
$$

and it can be easily checked that $M$ and $M^{\prime}$ are related by

$$
M={ }^{t} T M^{\prime} T
$$

with

$$
T=\frac{1}{\sqrt{2}}\left(\begin{array}{cccc}
0 & 1 & 0 & P \\
1 & 0 & P & 0 \\
0 & -1 & 0 & P \\
-1 & 0 & P & 0
\end{array}\right)
$$

If $\Psi$ is a solution of Helmholtz equation for the first billiard, given by (120) with constants specified by some vector $\mathbf{V}$, then $M \mathbf{V}=0$. Let $\Psi^{\prime}$ be the function defined on the second billiard by some constants given by the vector $\mathbf{V}^{\prime}=T \mathbf{V}$. Because of Eq. (123) the vector $\mathbf{V}^{\prime}$ verifies $M^{\prime} \mathbf{V}^{\prime}=0$, and therefore $\Psi^{\prime}$ is a solution of Helmholtz equation for the second billiard. Since the relation between $\Psi$ and $\Psi^{\prime}$ is linear, the eigenenery is the same for both functions, and thus the billiards are isospectral.

\section{G. Eigenvalue statistics}

In order to further characterize properties of the distribution of eigenvalues of the Laplacian, a commonly used function is the level spacing distribution function $P$. It is defined as the probability density distribution of the spacings between nearest-neighbour energy levels. That is, if we set $s_{i}=E_{i+1}-E_{i}$, the nearest-neighbour spacing distribution is defined by

$$
P(s) d s=\frac{1}{N} \sharp\left\{i ; s \leq s_{i} \leq s+d s\right\}
$$


where we only consider $N$ eigenvalues around a given energy $E$. In order to be able to compare statistics, the function $P(s)$ is chosen to be normalized by

$$
\int_{0}^{\infty} P(s) d s=1 \quad \text { and } \quad \int_{0}^{\infty} s P(s) d s=1
$$

For each class of classical systems described in section .D, conjectures relating the quantum behaviour to the classical properties of systems have been proposed. For integrable billiards, Berry and Tabor conjectured (Berry and Tabor, 1977) that the energy levels behave like independent uniformly distributed random variables, that is a Poisson distribution. The nearest-neighbour level spacing should thus be given by

$$
P(s)=e^{-s}
$$

Chaotic systems are so complex that a natural idea is to replace all parameters and variables appearing in its description by random variables. To study properties of chaotic systems, the basic idea is to replace the Hamiltonian of the system, which is out of reach analytically, by a matrix whose coefficients are independent Gaussian random variables. This random matrix theory (RMT) has been introduced by Wigner and Dyson (see (Porter, 1965) for a review on the seminal papers, and (Guhr et al., 1998) for a recent review on RMT). Three kinds of ensembles of random matrices (Gaussian orthogonal ensemble GOE, gaussian unitary ensemble GUE or Gaussian symplectic ensemble GSE) can be defined, according to the symmetries of the system that has to be described. Matrices are drawn from these ensembles with a Gaussian probability measure which is invariant under transformations corresponding to classical symmetries (i.e. under respectively orthogonal, unitary or symplectic transformations). Bohigas, Giannoni and Schmit conjectured (Bohigas et al., 1984) that systems which are invariant under time reversal symmetry are well described by GOE matrices, and by GSE matrices if angular momentum is a half-integer if there is no rotational invariance. Generic systems should be described by GUE matrices (Bohigas et al., 1984)-(Wintgen, 1987). Wigner (see (Guhr et al., 1998)) has proposed to approximate the nearest-neighbour spacing distribution for $N \times N$-matrices by its value for $2 \times 2$-matrices. This quantity has much simpler expressions and numerically corresponds 
very accurately to the exact $N \times N$-result. The general form of this "Wigner surmise" is

$$
P(s)=a_{\beta} s^{\beta} e^{-b_{\beta} s^{2}},
$$

with $\beta=1,2$ or 4 according to the symmetry class of the problem; $a_{\beta}$ and $b_{\beta}$ are given by normalization conditions (126). In particular systems with time-reversal symmetry can be described by the spectrum of GOE matrices. The nearest-neighbour distribution reads

$$
P(s)=\frac{\pi}{2} s \exp \left(-\frac{\pi}{4} s^{2}\right) .
$$

For intermediate systems, numerical results show that the nearest-neighbour spacing distribution behaves like a Wigner-Dyson distribution for small values of its argument, that is

$$
P(s) \sim_{s \rightarrow 0} s^{\alpha}, \quad \alpha>0,
$$

while it decreases exponentially at infinity, like Poisson distribution (127):

$$
P(s) \sim_{s \rightarrow \infty} \exp (-a s) .
$$

Another useful quantity used to characterize spectra of billiards is the spectral rigidity $\bar{\Delta}_{3}(L)$, defined in (Mehta, 1990) by par

$$
\bar{\Delta}_{3}(L)=\left\langle\min _{A, B \in \mathbb{R}} \frac{1}{L} \int_{-L / 2}^{L / 2}(N(E+\epsilon)-A-B \epsilon)^{2} d \epsilon\right\rangle .
$$

It measures the deviation of the density of states from a straight line, on an interval $[E-$ $L / 2, E+L / 2]$. Berry showed in (Berry, 1985) that for a generic integrable system

$$
\bar{\Delta}_{3}(L)=L / 15, \quad L \ll L_{\max }
$$

(with $L_{\max }=k t_{H} / T_{\min }, T_{\min }$ being the period of the shortest orbit and $t_{H}$ heiseberg time), and a non-universal saturation for $L \gg L_{\max }$. On the other hand, for a system which is 
invariant by time-reversal symmetry and with unstable periodic orbits

$$
\bar{\Delta}_{3}(L) \simeq(\ln L) / \pi^{2}-0.00695, \quad L \ll L_{\max }
$$

and non-universal saturation for $L \gg L_{\max }$.

As explained in section I, the shape of the building block can be varied as desired. Examples of chaotic pairs, or pseudointegrable pairs, or pairs with a fractal boundary can pe produced. Eigenvalue statistics for the pair of Fig. 1 have been studied numerically in (Wu et al., 1995), based on the first 598 energy levels. Removing the 78 triangular states, it was observed that $P(s)$ agrees with the nearest-neighbour distribution for GOE matrices given by Eq. (129). Computation of $\bar{\Delta}_{3}$ showed that it is also of GOE type for these billiards.

\section{H. Nodal domains}

Nodal lines for two-dimensional billiards are one-dimensional curves on which eigenfunctions vanish. Nodal domains are connected regions of the billiard where an eigenfunction has a constant sign. A theorem by Courant (Courant and Hilbert, 1953) states that the $n$-th eigenfunction $\Psi_{n}$ has at most $n$ nodal domains. The number $\nu_{n}$ of nodal domains in $\Psi_{n}$ can be further estimated (Pleijel, 1956). Let us define a rescaled nodal-domain number $\xi_{n}=\nu_{n} / n \in[0,1]$. If $j_{1}$ is the first zero of the Bessel function $J_{0}$, then $\lim _{n \rightarrow \infty} \xi_{n} \leq\left(2 / j_{1}\right)^{2}$. It has been shown that the limit distribution of $\xi_{n}$, defined by

$$
P(\xi)=\lim _{E \rightarrow \infty}\left\langle\delta\left(\xi-\xi_{n}\right)\right\rangle_{E_{n} \in[E, E+g E]}
$$

for some fixed $g>0$, has universal features.

For some instances of isospectral pairs, such as flat tori in $\mathbb{R}^{n}$ with $n \geq 4$ (Gnutzmann et al., 2005) (see also (Levitin et al., 2006)), it was conjectured that two isospectral domains produce a different number of nodal domains (domains separated by nodal lines where $\Psi=0$ ). Heuristic arguments as well as numerical investigations were collected in (Gnutzmann et al., 2005) to support this conjecture. 


\section{EXPERIMENTAL AND NUMERICAL INVESTIGATIONS}

Although isospectrality is proven on mathematical grounds, the knowledge of exact eigenvalues and eigenfunctions can not be obtained analytically for such systems. Experimental as well as numerical simulations have occured very early in the history of billiards. In 1909, in the Bulletin international de l'Académie des sciences de Cracovie, Stanislas Zaremba proposed a way of "numerically" calculating solutions of the Dirichlet and Neumann problem at a given point (Zaremba, février 1909). Usual numerical methods to compute eigenvalues and eigenfunctions in polygonal billiards are based on the so-called "method of particular solutions", or FHM as it was introduced by Fox, Henrici and Moler in (Fox et al., 1967). At a diffracting corner with angle $\pi \alpha$, a wavefunction $\Psi$ admits a "corner" decomposition into Bessel functions valid at a distance less than the nearest diffracting corner. In polar coordinates centred around the corner $\pi \alpha$ this decomposition reads

$$
\Psi(r, \theta)=\sum_{k} a_{k} J_{k \alpha}(k r) \sin (k \alpha \theta)
$$

where $J_{\nu}$ are Bessel functions of the first kind, and $k=\sqrt{E}$. The sine function in (136) ensures that the function $\Psi(r, \theta)$ is zero on the boundary edges connected to corner $\pi \alpha$. The idea of FHM is to require that $\Psi$ also vanish on the rest of the boundary at a finite number of points, and to truncate the sum (136). This gives a system of $m$ linear equations, which admits a non-zero solution $\left\{a_{k}, 1 \leq k \leq m\right\}$ if and only if the matrix corresponding to this linear system is singular. The FHM method therefore consists in varying the energy $E$ and tracking the singularities of the matrix $M$.

Unfortunately for more than one diffracting corner it becomes virtually impossible to track singularities, all the more since in various circomstances FHM fails to converge when the number of terms included in (136) is increased. Even for the paradigmatic pair with halfsquare base shape (Fig. 1), which is one of the simplest isospectral billiards, each pair has four diffractive angles: two $3 \pi / 2$ and two $3 \pi / 4$ angles, and FHM fails to give eigenvalues with a good accuracy. This is why attention has been focused on physical experiments. All known pairs of isospectral billiards are built on the same principle as the "historical" pair $7_{3}$ of Fig. 1. As has been explained in section I any initial building block possessing three sides along which to unfold the block can be used to construct an isospectral pair. 
In particular, the properties of the resulting pair will depend strongly on the choice of the initial building block. Physicists have mainly concentrated on the paradigmatic example of Fig. 1. The focus has mainly be put on this pair, which allows to make comparisons between the different approaches.

In this section we review both experimental and numerical investigations which give an insight into the behaviour of eigenvalues and eigenfunctions for isospectral billiards.

\section{A. Numerical investigations}

1. Numerical computations of the spectrum and eigenfunctions by a mode-matching method

Numerical approaches to the study of isospectrality for the billiards of Fig. 1 have followed the experiments of Sridhar and Kudrolli. Various approaches have been used in order to solve the Helmholtz equation $(\Delta+E) \Psi=0$ with Dirichlet boundary conditions inside the billiards. The first numerical results were obtained by Wu, Sprung and Martorell and reported in (Wu et al., 1995). Using the mode-matching method described in section V.F.2, eigenvalues of the billiard are the values for which the determinant of the matrix $M$, given by (121), vanishes. The results obtained by this method are displayed in column 2 of VI.B.2. As expected, both billiards yield the same values. Wu et al. (Wu et al., 1995) compared their results to results obtained by a finite-difference method consisting in discretizing the Laplacian $\Delta$. This finite-difference method gives the results displayed in column 1 of Fig. VI.B.2 (the numerical results are again exactly the same for both billiards). As a check for the validity of this approach, one can identify the eigenvalues of triangular states. Lowest-energy triangular state are expected to have eigenenergies equal to $5 \pi^{2} / d^{2}$ and $10 \pi^{2} / d^{2}$. As one can see in Table VI.B.2, these eigenvalues respectively correspond to the 9 th and the 21st mode, consistantly with Table V.F.1.

2. Numerical computations by expansion of eigenfunctions around the corners, with domaindecomposition method

The main drawback of the mode-matching method of (Wu et al., 1995) is the fact that one has to know analytic solutions of the Helmholtz equation on subdomains of the bil- 
liard. T.A. Driscoll, in (Driscoll, 1997), uses a numerical method based on an algorithm by Descloux and Tolley (Descloux and Tolley, 1983), particularly suited to treating the case of polygonal billiards. The idea is again to decompose the billiard into domains, each domain $\mathcal{D}_{i}$ containing only one diffracting angle $a_{i}$. On each domain the restriction of the eigenfunction $\Psi$ is supposed to be some $\Psi_{i}$ that admits a Bessel function expansion around corner $a_{i}$, according to (136). Truncating this expansion to some finite order, the problem reduces to finding the coefficients of the expansion for the $\Psi_{i}$. Mode-matching numerically leads to undesired singularities. Instead, (Descloux and Tolley, 1983) use an algorithm minimizing a function that measures discrepancies between the $\Psi_{i}$ and between their derivatives at the boundaries between subdomains. Improvement of this algorithm allowed Driscoll to obtain the first 25 eigenvalues for both billiards of Fig. 1 with an accuracy of up to 12 digits. Betcke and Trefethen (Betcke and Trefethen, 2005) use a modified method of particular solutions using 140 expansion terms at each singular corner, 140 boundary points on each side of the polygon, and 50 interior points to obtain following estimates for the first three eigenvalues: $2.537943999798,3.65550971352$ and 5.17555935622 .

\section{B. Experimental realizations}

\section{Electromagnetic waves in metallic cavities}

Many experimental studies have been carried out on chaotic quantum billiards to check the various properties conjectured analytically for chaotic systems (Bohigas et al., 1984). One commonly used method is based on the correspondence between the stationary Schrödinger equation and Helmholtz equation for electromagnetic waves in two dimensions (which is also the equation obeyed by vibrating plates). The experiments are carried out by sending electromagnetic microwaves into a cylindrical copper cavity. The height $h$ of the cavity is small, and the two other dimensions are shaped according to the desired billiards to investigate. For wavelengths $\lambda>2 h$, i.e. frequences below $\nu_{0}=c / 2 h$, all modes obey the two-dimensional wave equation $\left(\Delta+k^{2}\right) \Psi=0$. The $E_{z}$ component of the electric field plays the role of the quantum wave, and vanishes on the boundary. Probes allow to send an elecromagnetic wave in the cavity and to measure the transmission spec- 
trum. In particular, eigenvalues correspond to resonances in the transmission spectrum. Various choices of the probe locations ensure that no resonance is missed.

If $\mathcal{A}$ is the area of the cavity, the number of resonances below $\nu_{0}$ is approximately given by $\frac{\mathcal{A}}{4 \pi}(\pi / h)^{2}$. But the quality factor of the cavity is proportional to $h$; therefore one has to find a compromise between a high quality factor and a large number of resonances.

Measurements of the intensity of the wave function (or here the electric field) were achieved by the perturbation body method (Sridhar et al., 1992): the resonance frequency of the cavity is shifted by the presence of a small metallic body inside the cavity. This shift is a function of the square of the electric field at the point of the metallic body.

The first experimental investigation of isospectral billiards was realized at Northeastern University, Boston, by Srinivas Sridhar and Arshad Kudrolli (Sridhar and Kudrolli, 1994). Sridhar and coworkers had carried out various studies on chaotic quantum billiards, such as Sinai billiard (a square billiard with a circular obstacle in the interior) or Bunimovitch stadium-shaped billiard, observing the scarring of eigenfunctions (Sridhar, 1991) or localization phenomena (Sridhar and Heller, 1992) for such billiards. The experiments aimed at investigating isospectrality were realized on cavities having the shape of the isospectral pair of Fig. 1.

Experimentally, each cavity has 9 rectangular sides. The base shape is an isosceles rectangular triangle (a half-square) whose smaller size is $d=76 \mathrm{~mm}$ (3 in.) long. The height of the cavity is $h=6.3 \mathrm{~mm}$ ( $\simeq 0.25 \mathrm{in}$.), so that microwaves at frequencies below $\nu_{0}=25 \mathrm{GHz}$ are actually two-dimensional. Measurements carried out to obtain the 54 lowest eigenvalues showed that, as expected, the eigenvalues of the two cavities are equal. Relative discrepancies of 0.01 to $0.2 \%$ between pairs of eigenvalues were found. These discrepancies and the width of the resonances were assumed to be caused by imperfections due to assembly of the pieces forming the cavity. This experiment also allowed to get an insight on the properties of eigenvalues of isospectral pairs. It was checked that the eigenvalues found experimentally agree with the Weyl formula (71) for the integrated density of states:

$$
\mathcal{N}(E) \simeq \frac{\mathcal{A}}{4 \pi} E-\frac{\mathcal{L}}{4 \pi} \sqrt{E}+K
$$

For the choice $d=3$ in. one gets an area $\mathcal{A}=31.5$ in. $^{2}$ and a perimeter $\mathcal{L}=27$ in.; the constant $\mathcal{K}$ is given by (78) and yields $\mathcal{K}=5 / 12$. It was observed that, at least for 
the lowest eigenvalues no degeneracy occured. By measuring the electric field inside the cavity, some of the lowest eigenfunctions were obtained. The results for the 10 first eigenvalues are displayed in Table VI.B.2. It is funny to note that these pairs of eigenfunctions look quite different, although they possess the same eigenvalues. It was checked that one eigenfunction could be deduced from the other by transplantation. The particular case of the 9-th mode, which is a triangular state, is well reproduced. Indeed, as shown in Table VI.B.2, the measured 9-th eigenvalue is very close to its theoretical value $E=5 \pi^{2} / d^{2}$.

\section{Transverse vibrations in vacuum for liquid crystal smectic films}

Another experimental realization of Kac's membranes was achieved by using liquid crystal films in a smectic phase, spanned on a shape of the form of the isospectral billiard to be considered (Even and Pieranski, 1999). First, the shapes were etched in circular stainlesssteel wafers of diameter $4 \mathrm{~cm}$ and thickness $125 \mu \mathrm{m}$. The smectic film is then drawn on the shape, and after a few hours it reaches an equilibrium with uniform thickness $e$ of several hundred nm (corresponding to a few dozens monomolecular layers) over the whole surface. The whole experiment is set into vacuum. The film then obeys the wave equation

$$
\gamma \Delta z=\rho e \frac{\partial^{2} z}{\partial t^{2}}
$$

where $\gamma$ is the intrinsic tension of the film (in the experiments $\gamma \sim 5.10^{-2} \mathrm{~N} / \mathrm{m}$ ), and $\rho$ the density, with a vertical displacement $z$ vanishing on the border. The film is excited by a voltage applied by an electrode under the film, and the amplitude and phase of its oscillations are measured by sending a laser beam and measuring its deviations by a photodiode. The signal detected is proportional to the height of the film at the position of the electrode. The frequency of the excitation is varied from a few $\mathrm{Hz}$ to several $\mathrm{kHz}$, and eigenfrequencies correspond to resonance peaks. Displacing the electrode over the whole shape allows to reconstruct eigenmodes.

The experiment was carried on isospectral billiards with an isosceles triangular base shape: two angles $\beta=\gamma$ are equal, while the third one is varied from $\alpha=67.5^{\circ}$ to $\alpha=97.5^{\circ}$. The angle $\alpha=90^{\circ}$ corresponds to the example of Fig. 1. The first 30 modes for both shapes were measured. The average relative difference between two eigenvalues for a given mode is $0.3 \%$, which is within the estimated experimental error of order $0.5 \%$. For 
the right angle triangle $\left(\alpha=90^{\circ}\right)$ the modes are to be compared with other numerical or experimental results. Data for the 10 first eigenvalues of the $\alpha=90^{\circ}$ billiards were given in (Even and Pieranski, 1999) and are displayed in Table VI.B.2. When the parameter $\alpha$ is varied, there is an avoided crossing between eigenvalues of the 8th and the 9th mode. Since the 9th mode is a triangular mode (see section V.F.1) and the 8th is not, the coupling between these two modes necessarily comes from experimental imperfections.

This experiment has also been tested on a billiard where the gluing scheme of the base triangles is modified. Namely, tile $E$ in Fig. 11 left is flipped around the line $x=5 d / 2$. This leads to a significantly different spectrum. In particular, the triangular modes are no longer eigenstates of such a billiard. Again this is a check that the way the tiles are glued together, according to the rules constructed from finite projective spaces or from Sunada triples, is of primary importance for isospectrality. 


\begin{tabular}{|c|c|c|c|c|c|c|}
\hline rank & Finite differences & Mode matching & \multicolumn{2}{|c|}{ Electromagnetic waves } & \multicolumn{2}{|c|}{ Smectic films (relative values) } \\
\hline 1 & 1.028936 & 1.028535 & 1.02471 & 1.02481 & 1.000000 & 1.000000 \\
\hline 2 & 1.481865 & 1.481467 & 1.46899 & 1.47194 & 1.438000 & 1.430000 \\
\hline 3 & 2.098249 & 2.097467 & 2.08738 & 2.08831 & 2.040000 & 2.027000 \\
\hline 4 & 2.649715 & 2.649547 & 2.64079 & 2.63985 & 2.571000 & 2.548000 \\
\hline 5 & 2.938176 & 2.937434 & 2.93297 & 2.92949 & 2.854000 & 2.823000 \\
\hline 6 & 3.732689 & 3.732334 & 3.72695 & 3.71892 & 3.623000 & 3.570000 \\
\hline 7 & 4.295193 & 4.294728 & 4.28393 & 4.28388 & 4.184000 & 4.153000 \\
\hline 8 & 4.677665 & 4.677532 & 4.67021 & 4.66917 & 4.554000 & 4.507000 \\
\hline 9 & 5.000002 & 5.000000 & 4.98838 & 4.98531 & 4.861000 & 4.811000 \\
\hline 10 & 5.291475 & 5.290275 & 5.27908 & 5.27278 & 5.150000 & 5.095000 \\
\hline 11 & 5.801531 & 5.801138 & 5.78755 & 5.78371 & & \\
\hline 12 & 6.433894 & 6.432156 & 6.41357 & 6.43781 & & \\
\hline 13 & 6.866260 & 6.866226 & 6.84891 & 6.84718 & & \\
\hline 14 & 7.159802 & 7.159343 & 7.15242 & 7.16045 & & \\
\hline 15 & 7.694737 & 7.692417 & 7.67783 & 7.70604 & & \\
\hline 16 & 8.463655 & 8.463257 & 8.44285 & 8.45947 & & \\
\hline 17 & 8.613536 & 8.611169 & 8.57859 & 8.62220 & & \\
\hline 18 & 9.012405 & 9.010349 & 8.99495 & 8.97209 & & \\
\hline 19 & 9.609968 & 9.609791 & 9.60312 & 9.59562 & & \\
\hline 20 & 9.921131 & 9.921040 & 9.92583 & 9.93689 & & \\
\hline 21 & 10.000008 & 10.000000 & 10.00330 & 10.03932 & & \\
\hline 22 & 10.571020 & 10.569736 & 10.55227 & 10.55740 & & \\
\hline 23 & 11.066916 & 11.065727 & 11.09578 & 11.10035 & & \\
\hline 24 & 11.419551 & 11.418850 & 11.41874 & 11.40569 & & \\
\hline 25 & 11.984650 & 11.984080 & 11.99364 & 11.98033 & & \\
\hline
\end{tabular}

Table II Comparison between the first eigenvalues $E_{i}$ of the isospectral pair obtained by various methods, expressed in units of $\pi^{2} / d^{2}$. The 9-th mode corresponds to the triangular mode: its normalised eigenvalue is expected to be equal to 5 . (The conversion from frequencies to lengths is done assuming vacuum in the cavity; (Wu et al., 1995) give the values for electromagnetic cavities with a factor 1.0006 corresponding to the presence of air in the cavity.)

\section{TRANSPLANTATION}

The aim of this section is to describe the concept of transplantation. This concept was presumably first introduced by P. Bérard - see his papers (Bérard, 1992, 1993). It will include the geometric aspect behind Kac theory.

\section{A. Graphs and billiards by tiling}

Tiling. All known isospectral billiards can be obtained by unfolding polygonal-shaped tiles, but essentially one can only consider triangles. We call such examples isospectral 
Euclidean TI-domains. The way the tiles are unfolded can be specified by three permutation $d \times d$-matrices $M^{(\mu)}, 1 \leq \mu \leq 3$ and $d \in \mathbb{N}$, associated with the three sides of the triangle:

- $M_{i j}^{(\mu)}=1$ if tiles $i$ and $j$ are glued by their side $\mu$;

- $M_{i i}^{(\mu)}=1$ if the side $\mu$ of tile $i$ is the boundary of the billiard, and

- 0 otherwise.

The number of tiles is, of course, $d$. Call the matrices $M^{(\mu)}$ "adjacency matrices".

One can sum up the action of the $M^{(\mu)}$ in a graph with coloured edges: each copy of the base tile is associated with a vertex, and vertices $i$ and $j, i \neq j$, are joined by an edge of colour $\mu$ if and only if $M_{i j}^{(\mu)}=1$. In the same way, in the second member of the pair, the tiles are unfolded according to permutation matrices $N^{(\mu)}, 1 \leq \mu \leq 3$. We call such a coloured graph an involution graph for reasons to be explained later in this section. If $D$ is a Euclidean TI-domain with base tile a triangle, and $\mathfrak{M}=\left\{M^{(\mu)} \| \mu \in\{1,2,3\}\right\}$ is the set of associated permutation matrices (or, equivalently, the associated colouring), denote by $\Gamma(D, \mathfrak{M})$ the corresponding involution graph.

The following proposition is easy but fundamental (Thas, 2007).

Proposition VII.1 Let $D$ be a Euclidean TI-domain with base tile a triangle, and let $\mathfrak{M}=$ $\left\{M^{(\mu)} \| \mu \in\{1,2,3\}\right\}$ be the set of associated permutation matrices. Then

$$
\sum_{\mu=1}^{3} M^{(\mu)}-\Delta\left(\sum_{\mu=1}^{3} M^{(\mu)}\right)
$$

is the adjacency matrix of $\Gamma(D, \mathfrak{M})$, where $\Delta^{M}=\Delta\left(\sum_{\mu=1}^{3} M^{(\mu)}\right)$ is defined by $\Delta_{i i}^{M}=\left(\sum_{\mu=1}^{3} M^{(\mu)}\right)_{i i}$ for all $i$, and $\Delta_{i j}^{M}=0$ if $i \neq j$.

Transplantability. Two billiards are said to be transplantable if there exists an invertible matrix $T$ - the transplantation matrix - such that

$$
T M^{(\mu)}=N^{(\mu)} T \text { for all } \mu \text {. }
$$



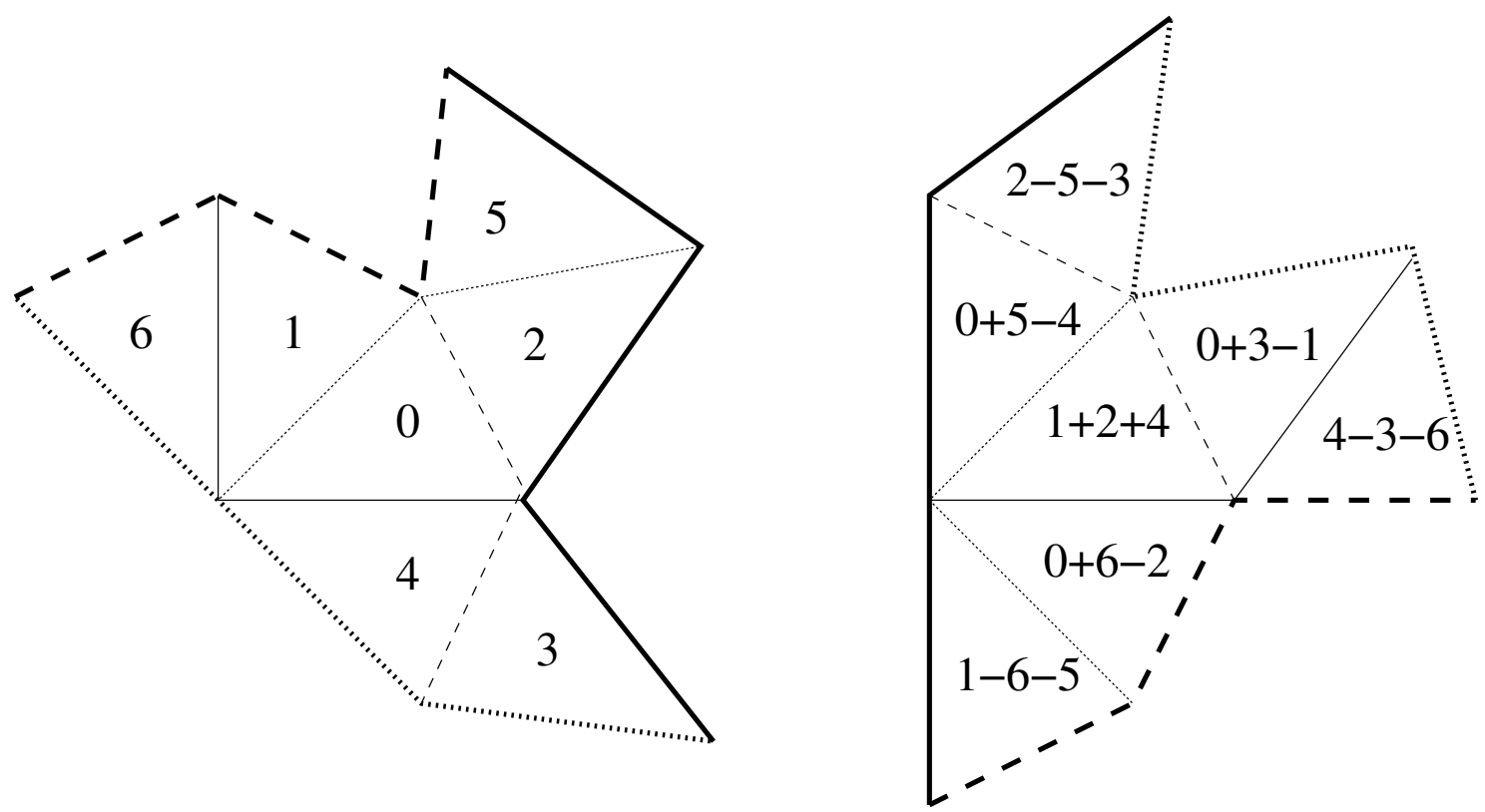

Figure 13 Two isospectral billiards with a triangular base shape on 7 tiles.

If the matrix $T$ is a permutation matrix, the two domains would just have the same shape. One can show that transplantability implies isospectrality. For, if $\Psi_{1}$ is an eigenfunction of the first billiard and $\Psi_{1}^{(i)}$ is its restriction to triangle $i$, then one can build an eigenfunction $\Psi_{2}$ of the second billiard by taking

$$
\Psi_{2}^{(i)}=\sum_{j} T_{i j} \Psi_{1}^{(j)}
$$

In the next section, we will discuss in detail the celebrated example found in (C. Gordon and Wolpert, 1992) by Buser et al. We will show isospectrality by following the method of Buser et al. (Buser et al., 1994). As the reader will notice, this will in fact be a down-to-earth approach/example of transplantability.

\section{B. The example of Gordon et al.}

In this section we analyse the first known example of a pair of simply connected compact isospectral but non-congruent Euclidian domains. The pair was found by C. Gordon, D. Webb and S. Wolpert in their paper "Isospectral plane domains and surfaces via Riemannian orbifolds" (C. Gordon and Wolpert, 1992) (Invent. Math. 110 (1992), 1-22). 
We follow the very transparent approach of Buser et al. (Buser et al., 1994) to show isospectrality.

Let $\lambda$ be any real number, and $f$ any eigenfunction of the Laplacian with eigenvalue $\lambda$ for the Dirichlet problem corresponding to the left-hand propeller (in Fig. VII.B). Let $f_{0}, f_{1}, \ldots, f_{6}$ denote the functions obtained by restricting $f$ to each of the 7 triangles of the left-hand propeller, as indicated on the left in Fig. VII.B.

Notation. For brevity, we write $\mathbf{i}$ for $f_{i}$.

The Dirichlet boundary condition is that $f$ must vanish on each boundary-segment. Using the reflection principle, this is equivalent to the assertion that $f$ would go into $-f$ if continued as a smooth eigenfunction across any boundary-segment. (More precisely it goes into $f \circ \sigma$ where $\sigma$ is the reflection on the boundary segment.)

On the right in Fig. VII.B, we show how to obtain from $f$ another eigenfunction of eigenvalue $\lambda$, this time for the right-hand propeller. In the central triangle, we put the function $1+2+4$. By this we mean the function $f_{1} \circ \tau_{1}+f_{2} \circ \tau_{2}+f_{4} \circ \tau_{4}$ where for $k=1,2,4, \tau_{k}$ is the isometry from the central triangle of the right-hand propeller to the triangle labelled $k$ on the left-hand propeller. Now we see from the left-hand side that the functions $1,2,4$ continue smoothly across dotted lines into copies of the functions $\mathbf{0 , 5}, \mathbf{- 4}$ respectively, so that their sum continues into $\mathbf{0}+\mathbf{5}-\mathbf{4}$ as shown. (The reader can check in a similar way that this continues across a solid line to $4-5-\mathbf{0}$ (its negative), and across a dashed line to $2-5-3$, which continues across either a solid or dotted line to its own negative.) These assertions, together with the similar ones obtained by symmetry (i.e. cyclic permutation of the arms of the propellers), are enough to show that the transplanted function is an eigenfunction of eigenvalue $\lambda$ that vanishes along each boundary segment of the right-hand propeller.

So we have defined a linear map which for each $\lambda$ takes the $\lambda$-eigenspace for the left-hand propeller to the $\lambda$-eigenspace for the right-hand one. This is easily checked to be a nonsingular map, and so the dimension of the eigenspace on the right-hand side is larger or equal the dimension on the left-hand side. Since the same transplantation may also be applied in the reversed direction the dimensions are equal. This holds for each $\lambda$, and so 
the two propellers are Dirichlet isospectral.

In fact they are also Neumann isospectral, as can be seen by a similar transplantation proof obtained by replacing every minus sign in the above by a plus sign. (Going from Neumann to Dirichlet is almost as easy: just color the triangles on each side alternately black and white, and attach minus signs on the right to function elements that have moved from black to white or vice versa.)

\section{The other known examples}

A similar technique as in the previous section allowed Buser et al. (Buser et al., 1994) to show that the series of billiard pairs they produced in the aforementioned paper are indeed isospectral. All these pairs are listed in an appendix to this paper; they were first found by searching for suitable Sunada triples, and then verified to be isospectral (in the plane) by the transplantation method. We also refer to (Okada and Shudo, 2001b) for a further discussion on the subject of this section.

\section{Euclidean TI-domains and their involution graphs}

The following question was posed in (Thas, 2007):

Question. Let $\left(D_{1}, D_{2}\right)$ be a pair of nonisometric isospectral Euclidean TI-domains, and let $\Gamma\left(D_{1}\right)=\Gamma\left(D_{1},\left\{M^{(\mu)} \| \mu \in\{1,2,3\}\right\}\right)$ and $\Gamma\left(D_{2}\right)=\Gamma\left(D_{2},\left\{N^{(\mu)} \quad \| \mu \in\{1,2,3\}\right\}\right)$ be the corresponding involution graphs. Are $\Gamma\left(D_{1}\right)$ and $\Gamma\left(D_{2}\right)$ cospectral?

Note that one does not ask the domains to be transplantable.

We now show that, maybe surprisingly, the answer is "yes" when the domains are transplantable. The proof is taken from (Thas, 2007). 
Proof. Define, for $\mu=1,2,3, M_{*}^{(\mu)}$ as the matrix which has the same entries as $M^{(\mu)}$, except on the diagonal, where it only has zeroes. Define matrices $N_{*}^{(\mu)}$ analogously. Suppose that $T M^{(\mu)} T^{-1}=N^{(\mu)}$ for all $\mu$.

Note the following properties:

- $M_{*}^{(\mu)}$ and $N_{*}^{(\mu)}, \mu=1,2,3$, are symmetric $(0,1)$-matrices, with at most one 1 entry on each row;

- $\left[M_{*}^{(\mu)}\right]^{m}=M_{*}^{(\mu)}$ if the natural number $m$ is odd and $\left[M_{*}^{(\mu)}\right]^{m}=\mathbb{I}_{M}^{(\mu)}$, where $\left[\mathbb{I}_{M}^{(\mu)}\right]_{i i}=1$ if there is a 1 on the $i$-th row of $M_{*}^{(\mu)}$, and 0 otherwise, if $m$ is even, $\mu=1,2,3$, and similar properties hold for the $N_{*}^{(\mu)}$;

- $\operatorname{Tr}\left(M_{*}^{(i)} M_{*}^{(j)}\right)=\operatorname{Tr}\left(M_{*}^{(j)} M_{*}^{(i)}\right)=0$ for $i \neq j$ and $\operatorname{Tr}\left(N_{*}^{(i)} N_{*}^{(j)}\right)=\operatorname{Tr}\left(N_{*}^{(j)} N_{*}^{(i)}\right)=0$ for $i \neq j$;

- $\operatorname{Tr}\left(M_{*}^{(i)} M_{*}^{(j)} M_{*}^{(k)}\right)$ and $\operatorname{Tr}\left(N_{*}^{(i)} N_{*}^{(j)} N_{*}^{(k)}\right)$ are independent of the permutation $(i j k)$ of (123) (this is because the individual matrices are symmetric);

- the value of all traces in the previous property is 0 (note that if $\{i, j, k\}=\{1,2,3\}$, such a trace equals 0 since the existence of a nonzero diagonal entry of $M_{*}^{(i)} M_{*}^{(j)} M_{*}^{(k)}$, respectively $N_{*}^{(i)} N_{*}^{(j)} N_{*}^{(k)}$, implies $\Gamma\left(D_{1}\right)$, respectively $\Gamma\left(D_{2}\right)$, to have closed circuits of length 3);

- $\sum_{i=1}^{3} M_{*}^{(\mu)}=\sum_{\mu=1}^{3} M^{(\mu)}-\Delta\left(\sum_{\mu=1}^{3} M^{(\mu)}\right)$ and $\sum_{i=1}^{3} N_{*}^{(\mu)}=\sum_{\mu=1}^{3} N^{(\mu)}-$ $\Delta\left(\sum_{\mu=1}^{3} N^{(\mu)}\right)$.

Put $A=\sum_{i=1}^{3} M_{*}^{(\mu)}$, the adjacency matrix of $\Gamma\left(D_{1}\right)$, and $B=\sum_{i=1}^{3} N_{*}^{(\mu)}$, the adjacency matrix of $\Gamma\left(D_{2}\right)$.

Consider a natural number $n \in \mathbb{N}_{0}$. Then keeping the previous properties in mind, it follows that

$$
\operatorname{Tr}\left(A^{n}\right)=\operatorname{Tr}\left(B^{n}\right)
$$

Whence by the following lemma (cf. (van Dam and Haemers, 2003, Lemma 1)) the adjacency matrices of $\Gamma\left(D_{1}\right)$ and $\Gamma\left(D_{2}\right)$ have the same spectrum. 


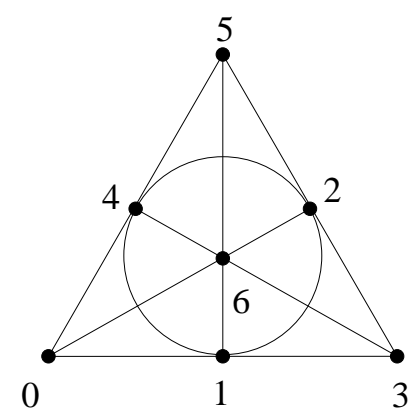

Figure 14 The Fano plane.

Lemma VII.2 Two $k \times k$-matrices $K$ and $K^{\prime}$ are cospectral if and only if $\operatorname{Tr}\left(K^{l}\right)=\operatorname{Tr}\left(K^{\prime l}\right)$ for $l=1,2, \ldots, n$.

\section{E. Finite projective geometry}

More details on the notions considered in this section can be found in (Hirschfeld, 1998).

Let $\mathbb{F}_{q}$ be the finite field with $q$ elements, $q$ a prime power, and denote by $V(n, q)$ the $n$-dimensional vectorspace over $\mathbb{F}_{q}, n$ a nonzero natural number. Define the $(n-1)$ dimensional projective geometry $\mathbf{P G}(n-1, q)$ over $\mathbb{F}_{q}$ as the geometry of all subspaces of $V(n, q)$ ordered by set inclusion.

Note that $\mathbf{P G}(n-1, q)$ is often called the "Desarguesian" or "classical" projective space.

The projective space $\mathbf{P G}(-1, q)$ is the empty set, and has dimension -1 .

A $d$ (-dimensional)-subspace of $\mathbf{P G}(n, q)$ contains $\left(q^{d+1}-1\right) /(q-1)$ points. In particular, $\mathbf{P G}(n, q)$ has $\left(q^{n+1}-1\right) /(q-1)$ points. It also has $\left(q^{n+1}-1\right) /(q-1)$ hyperplanes $(=$ $(n-1)$-dimensional subspaces).

Example. The Fano plane has 7 points, 7 lines, three points on each line ( $=$ three points "incident" with each line) and three lines through each point (= three lines "incident" with each point). 


\section{F. Axiomatic projective spaces}

A more synthetic way of defining projective spaces can be done by means of axioms as follows.

An axiomatic projective space is a set $\mathcal{P}$ (the set of points), together with a set of subsets of $\mathcal{P}$ (the set of lines) - all of which have at least three elements, and a symmetric incidence relation, satisfying these axioms:

- Each two distinct points $p$ and $q$ are incident with exactly one line.

- Axiom of Veblen: when $L$ contains a point of the line through $p$ and $q \neq p$ (different from $p$ and $q$ ), and of the line through $q$ and $r \neq q$ (different from $q$ and $r$ ), it also contains a point on the line through $p$ and $r$.

- There is a point $p$ and a line $L$ that are disjoint.

The last axiom is there to prevent degenerations.

If the number of points of the space is finite, we speak of a "finite projective space" (FPS). A subspace of the projective space is a subset $X$, such that any line containing two points of $X$ is a subset of $X$. The full space and the empty space are also considered as subspaces. The geometric dimension of the space is said to be $n$ if that is the largest number for which there is a strictly ascending chain of subspaces of the form

$$
\emptyset=X_{-1} \subset X_{0} \subset \cdots \subset X_{n}=\mathcal{P} \text {. }
$$

The following result is classical; it states that all axiomatic (finite) projective spaces of dimension at least three are essentially unique (they are of Desarguesian type). For dimension two, this result would be false, for non-Desarguesian examples are known - see (Hughes and Piper, 1973).

Theorem VII.3 A finite axiomatic projective space of dimension at least 3 is Desarguesian, so is isomorphic to a $\mathbf{P G}(n, q)$ for some natural number $n \geq 3$ and finite field $\mathbb{F}_{q}$.

We now take a closer look at (axiomatic) projective planes, for which we first provide the following equivalent definition. 
A finite axiomatic projective plane $\Pi$ of order $n$, where $n \in \mathbb{N}$, is a point-line incidence structure satisfying the following conditions:

(i) each point is incident with $n+1$ lines and each line is incident with $n+1$ points;

(ii) any two distinct lines intersect in exactly one point and any two distinct points lie on exactly one line.

One also traditionally requires that $n$ be $\geq 2$ to exclude the uninteresting cases of a single line and a point not on it $(n=-1)$, a single line and one point on it $(n=0)$, or the three vertices and three sides of a triangle $(n=1)$. This is equivalent to requiring that $\Pi$ contains an ordinary quadrangle (four points with no three on a line) as subgeometry. It is easily seen that a finite projective plane of order $n$ has $n^{2}+n+1$ points and $n^{2}+n+1$ lines.

The obvious examples of finite projective planes are the projective planes $\operatorname{PG}(2, q)$ over finite fields $\mathbb{F}_{q}$ as defined before. In this case the order $n=\left|\mathbb{F}_{q}\right|$ is a prime power, and in fact no examples of finite projective planes of non prime power order are known. A classical theorem of R. Moufang states that a finite projective plane is isomorphic to some $\mathbf{P G}(2, q)$ if and only if a certain configurational property corresponding to the classical theorem of Desargues is satisfied. Projective planes of this type are therefore often called Desarguesian, and since these correspond to planes coordinatized over finite fields, we also use this terminology for projective spaces of dimension $n \geq 3$, as already mentioned.

However, many finite projective planes are known which are not Desarguesian — see (Hughes and Piper, 1973).

\section{G. Automorphism groups}

An automorphism or collineation of a finite projective space is an incidence and type preserving bijection (so line set and point set are preserved) of the set of subspaces to itself. It can be shown that any automorphism of a $\mathbf{P G}(n, q), n \geq 3$, necessarily has the following form:

$$
\theta: \mathbf{x}^{T} \mapsto A\left(\mathbf{x}^{\sigma}\right)^{T}
$$


where $A \in \mathbf{G L}(n+1, q), \sigma$ is a field automorphism of $\mathbb{F}_{q}$, the homogeneous coordinate $\mathbf{x}=\left(x_{0}, x_{1}, \ldots, x_{n}\right)$ represents a point of the space (which is determined up to a scalar), and $\mathbf{x}^{\sigma}=\left(x_{0}^{\sigma}, x_{1}^{\sigma}, \ldots, x_{n}^{\sigma}\right)$ (recall that $x_{i}^{\sigma}$ is the image of $x_{i}$ under $\sigma$ ).

The set of automorphisms of a projective space naturally forms a group, and in case of $\mathbf{P G}(n, q), n \geq 3$, this group is denoted by $\mathbf{P} \boldsymbol{\Gamma} \mathbf{L}(n+1, q)$. The normal subgroup of $\mathbf{P} \Gamma \mathbf{L}(n+$ $1, q)$ which consists of all automorphisms for which the companion field automorphism $\sigma$ is the identity, is the projective general linear group, and denoted by $\operatorname{PGL}(n+1, q)$. So $\mathbf{P G L}(n+1, q)=\mathbf{G L}(n+1, q) / Z(\mathbf{G L}(n+1, q))$, where $Z(\mathbf{G L}(n+1, q))$ is the central subgroup of all scalar matrices of $\mathbf{G L}(n+1, q)$. Similarly one defines $\operatorname{PSL}(n+1, q)=$ $\mathbf{S L}(n+1, q) / Z(\mathbf{S L}(n+1, q))$, where $Z(\mathbf{S L}(n+1, q))$ is the central subgroup of all scalar matrices of $\mathbf{S L}(n+1, q)$ with unit determinant.

An elation of $\mathbf{P G}(n, q)$ is an automorphism of which the fixed points structure precisely is a hyperplane, or the space itself. A homology either is the identity, or it is an automorphism that fixes a hyperplane pointwise, and one further point not contained in that hyperplane.

\section{H. Involutions in finite projective space}

Let $\mathbf{P G}(n, q), n \in \mathbb{N} \cup\{-1\}$, be the $n$-dimensional projective space over the finite field $\mathbb{F}_{q}$ with $q$ elements, so that $q$ is a prime power; we have $|\mathbf{P G}(n, q)|=\frac{q^{n+1}-1}{q-1}$. (Note again that $\mathbf{P G}(-1, q)$ is the empty space.)

We discuss the different types of involutions that can occur in the automorphism group of a finite projective space (Segre., 1961).

- BAer Involutions. A Baer involution is an involution which is not contained in the linear automorphism group of the space, so that $q$ is a square, and it fixes an $n$-dimensional subspace over $\mathbb{F}_{\sqrt{q}}$ pointwise.

- Linear Involutions in Even Characteristic. If $q$ is even, and $\theta$ is an involution which is not of Baer type, $\theta$ must fix an $m$-dimensional subspace of $\mathrm{PG}(n, q)$ pointwise, with $1 \leq m \leq n \leq 2 m+1$. In fact, to avoid trivialities, one assumes that $m \leq n-1$. 
- Linear involutions in OdD Characteristic. If $\theta$ is a linear involution of PG $(n, q), q$ odd, the set of fixed points is the union of two disjoint complementary subspaces. Denote these by $\mathbf{P G}(k, q)$ and $\mathbf{P G}(n-k-1, q), k \geq n-k-1>-1 .^{1}$

We are now ready to explore a connection bewtween Incidence Geometry and Kac Theory.

\section{Transplantation matrices, projective spaces and isospectral data}

Suppose we want to construct a pair of isospectral billiards, starting from any polygonal base shape. The idea described in (Giraud, 2005a) is to start from the transplantation matrix $T$, and choose it in such a way that the existence of commutation relations

$$
T M^{(\mu)}=N^{(\mu)} T
$$

for some permutation matrices $M^{(\mu)}, N^{(\mu)}$ will be known a priori. As we will see, this is the case if $T$ is taken to be the incidence matrix of an FPS; the matrices $M^{(\mu)}$ and $N^{(\mu)}$ are then permutations on the points and the hyperplanes of the FPS.

An $(N, k, \lambda)$-symmetric balanced incomplete block design (SBIBD) is a set of $N$ points, belonging to $N$ subsets (or blocks) such that

- each block contains $k$ points;

- any two distinct points belong to exactly $\lambda$ blocks, and

- each point is contained in $k$ different blocks.

One can show that a $\mathbf{P G}(n, q)$ is an $(N, k, \lambda)$-SBIBD with $N=\left(q^{n+1}-1\right) /(q-1)$, $k=\left(q^{n}-1\right) /(q-1)$ and $\lambda=\left(q^{n-1}-1\right) /(q-1)$.

Example. The Fano plane is a $(7,3,1)$-SBIBD.

\footnotetext{
${ }^{1}$ We do not consider the possibility of involutions without fixed points, as they are not relevant for our purpose.
} 
The points and the blocks can be labeled from 0 to $N-1$. For any $(N, k, \lambda)-\operatorname{SBIBD}$ one can define an $N \times N$-incidence matrix $T$ describing to which block each point belongs. The entries $T_{i j}$ of the matrix are $T_{i j}=1$ if the point $j$ belongs to the line $i$, and 0 otherwise. The matrix $T$ verifies the relation

$$
T T^{T}=\lambda \mathbf{J}+(N-k) \lambda /(k-1) \mathbf{I},
$$

where $\mathbf{J}$ is the $N \times N$-matrix with all entries equal to 1 and $\mathbf{I}$ the $N \times N$ identity matrix. In particular, the incidence matrix of $\mathbf{P G}(n, q)$ verifies

$$
T T^{T}=\lambda \mathbf{J}+(k-\lambda) \mathbf{I}
$$

with $k$ and $\lambda$ as given above.

Example. The incidence matrix of the Fano plane corresponds to a labeling of the lines such that line the 0 contains the points $0,1,3$, and the line 1 contains the points 1,2 , etc.

Any permutation $\sigma$ on the points of an FPS can be written as a $d \times d$ permutation matrix $M$ defined by $M_{i \sigma(i)}=1$ and the other entries equal to zero.

If $M$ is a permutation matrix associated to a collineation, then there exists a permutation matrix $N$ such that

$$
T M=N T \text {. }
$$

In other words, (148) means that permuting the columns of $T$ (i.e. the hyperplanes of the space) under $M$ is equivalent to permuting the rows of $T$ (i.e. the points of the space) under $N$.

Let us consider an FPS $\pi$ with incidence matrix $T$. To each hyperplane in $\pi$ we associate a tile in the first billiard, and to each point in $\pi$ we associate a tile in the second billiard. If we choose permutations $M^{(\mu)}$ in the collineation group of $\pi$, then the commutation relation (148) will ensure that there exist permutations $N^{(\mu)}$ verifying 


$$
T M^{(\mu)}=N^{(\mu)} T
$$

These commutation relations imply transplantability, and thus isospectrality, of the billiards constructed from the graphs corresponding to $M^{(\mu)}$ and $N^{(\mu)}$.

If the base tile has $r$ sides, we need to choose $r$ elements $M^{(\mu)}, 1 \leq \mu \leq r$, in the collineation group of $\pi$. This choice is constrained by several factors:

- Since $M^{(\mu)}$ represents the reflexion of a tile with respect to one of its sides, it has to be an involution.

- In order that the billiards be connected, no point should be left out by the matrices $M^{(\mu)}$ - in other words, the graph associated to the matrices $M^{(\mu)}$ should be connected.

- Finally, if we want the base tile to be of any shape, there should be no loop in the graph.

Let us first assume we are looking for a pair of isospectral billiards with $d=\left(q^{3}-1\right) /(q-1)$ copies of a base tile having the shape of an $r$-gon, $r \geq 3$. We need to find $r$ involutions such that the associated graph is connected and without a loop. Such a graph connects $d$ vertices and thus requires $d-1$ edges. Since a collineation is a permutation, it has a cycle decomposition as a product of transpositions. For involutions with $s$ fixed points, there are $(d-s) / 2$ independent transpositions in this decomposition. Each transposition is represented by an edge in the graph. As a consequence, $q, r$ and $s$ have to fulfill the following condition:

$$
r\left(q^{2}+q+1-s\right) / 2=q^{2}+q
$$

More generally, we define "projective isospectral data" as triples $\left(\mathbf{P},\left\{\theta^{(i)}\right\}, r\right)$, where $\mathbf{P}$ is a finite projective space of dimension at least 2 , and $\left\{\theta^{(i)}\right\}$ a set of $r$ nontrivial involutions of $\mathbf{P}$, satisfying the following equation

$$
r(|\mathbf{P}|-\operatorname{Fix}(\theta))=2(|\mathbf{P}|-1),
$$


for some natural number $r \geq 3$. Here $\operatorname{Fix}(\theta)=\operatorname{Fix}\left(\theta^{(i)}\right)$ is a constant number of fixed points of $\mathbf{P}$ under each $\theta^{(i)}$, and $|\mathbf{P}|$ is the number of points of $\mathbf{P}$.

One can now generate all possible pairs of isospectral billiards whose transplantation matrix is the incidence matrix of a $\mathbf{P G}(2, q)$, with $r$ and $q$ restricted by the previous analysis. All pairs must have a triangular base shape $(r=3)$.

Using the classification of involutions for dimension 2, we examine the various cases.

LET $q$ BE EVEN AND NOT A SQUARE. Then any involution is an elation and therefore has $q+1$ fixed points. Therefore, $q$ and $r$ are constrained by the relation

$$
r q^{2} / 2=q^{2}+q
$$

The only integer solution with $r \geq 3$ and $q \geq 2$ is $(r=3, q=2)$. These isospectral billiards correspond to the Fano plane $\operatorname{PG}(2,2)$ and will be made of $d=7$ copies of a base triangle.

LET $q$ BE ODD AND NOT A SQUARE. Then any involution is a homology and therefore has $q+2$ fixed points. Therefore, $q$ and $r$ are constrained by the relation

$$
r\left(q^{2}-1\right) / 2=q^{2}+q
$$

The only integer solution with $r \geq 3$ and $q \geq 2$ is $(r=3, q=3)$. These isospectral billiards correspond to $\mathbf{P G}(2,3)$ and will be made of $d=13$ copies of a base triangle.

LET $q=p^{2}$ BE A SQUARE. Then any involution fixes all points in a Baer subplane PG $(2, p)$ and therefore has $p^{2}+p+1$ fixed points. Therefore, $p$ and $r$ are constrained by the relation

$$
r\left(p^{4}-p\right) / 2=p^{4}+p^{2}
$$

There is no integer solution with $r \geq 3$ and $q \geq 2$.

However, one can look for isospectral billiards with loops: this will require the base tile to have a shape such that the loop does not make the copies of the tiles come on top of each other when unfolded. If we tolerate one loop in the graph describing the isospectral 
billiards, then there are $d$ edges in the graph instead of $d-1$ and the equation for $p$ and $r$ becomes $r\left(p^{4}-p\right) / 2=p^{4}+p^{2}+1$, which has the only integer solution $(r=3, p=2)$. These isospectral billiards correspond to $\mathbf{P G}(2,4)$ and will be made of $d=21$ copies of a base triangle.

To summarize, we have that:

- The Fano plane $\mathbf{P G}(2,2)$ provides 3 pairs (made of 7 tiles).

- $\mathbf{P G}(2,3)$ provides 9 pairs (made of 13 tiles).

- $\mathbf{P G}(2,4)$ provides 1 pair (made of 21 tiles).

It turns out that the pairs obtained here are exactly those obtained in (Buser et al., 1994; Okada and Shudo, 2001b).

Now consider the space $\mathbf{P G}(3,2)$, which contains 15 points. The collineation group of $\mathbf{P G}(3,2)$ is the group $\mathbf{P G L}(4,2) \cong \mathbf{P} \mathbf{L}(4,2) \cong \mathbf{G L}(4,2)$. Generating (on a PC) all possible graphs from the 316 involutions, one obtains four pairs of isospectral billiards with 15 triangular tiles, which completes the list of all pairs found in (Buser et al., 1994; Okada and Shudo, 2001b).

For projective spaces of dimension 2 , we have the next result.

Theorem VII.4 (O. Giraud (Giraud, 2005a)) Let $\mathbf{P}=\mathbf{P G}(2, q)$ be the 2-dimensional projective space over the finite field $\mathbb{F}_{q}$, and suppose there exists projective isospectral data $\left(\mathbf{P},\left\{\theta^{(i)}\right\}, r\right)$. If $q$ is not a square, then $(r, q) \in\{(3,2),(3,3)\}$. If $q$ is a square, then there are no integer solutions of Equation (151).

So this method explicitly gives the transplantation matrix $T$ for all these pairs: each one is the incidence matrix of an FPS, and the transplantation matrix explicitly provides the mapping between eigenfunctions of both billiards. The inverse mapping is given by

$$
T^{-1}=\left(1 / q^{n-1}\right)\left(T^{T}-(\lambda / k) \mathbf{J}\right)
$$




\section{J. Generalized isospectral data}

In (Thas, 2006a) the next generalization was obtained for any dimension $n \geq 2$.

Theorem VII.5 (K. Thas (Thas, 2006a)) Let $\mathbf{P}=\mathbf{P G}(n, q)$ be the n-dimensional projective space over the finite field $\mathbb{F}_{q}$, and suppose there exists projective isospectral data $\left(\mathbf{P},\left\{\theta^{(i)}\right\}, r\right)$. Then $q$ cannot be a square. If $q$ is not a square, then $(r, n, q) \in$ $\{(3,2,2),(3, n, 3)\}$, where in the the case $(r, n, q)=(3, n, 3)$ each $\theta^{(i)}$ fixes pointwise a hyperplane, and also a point not in that hyperplane. However, this class of solutions only generates planar isospectral pairs if $n=2$.

Call a triple $\left(\mathbf{P},\left\{\theta^{(i)}\right\}, r\right)$, where $\mathbf{P}$ is a finite projective space of dimension at least 2 , and $\left\{\theta^{(i)}\right\}$ a set of $r$ nontrivial involutory automorphisms of $\mathbf{P}$, satisfying

$$
r(|\mathbf{P}|)-\sum_{j=1}^{r} \operatorname{Fix}\left(\theta^{(j)}\right)=2(|\mathbf{P}|-1),
$$

for some natural number $r \geq 3$, "generalized projective isospectral data".

These data were completely classified in (Thas, 2006b).

Theorem VII.6 (K. Thas (Thas, 2006b)) Let $\mathbf{P}=\mathbf{P G}(l, q)$ be the l-dimensional projective space over the finite field $\mathbb{F}_{q}, l \geq 2$, and suppose there exists generalized projective isospectral data $\left(\mathbf{P},\left\{\theta^{(i)}\right\}, r\right)$ which yield isospectral billiards. Then either $l=2$, the $\theta^{(i)}$ fix the same number of points of $\mathbf{P}$, and the solutions are as previously described, or $l=3, r=3$ and $q=2$, and again the examples are as before.

\section{K. The operator group}

Suppose $D$ is a Euclidean TI-domain on $d$ base triangles, and let $M^{(\mu)}, \mu \in\{1,2,3\}$, be the corresponding permutation $d \times d$-matrices. Define again involutions $\theta^{(\mu)}$ on a set $X$ of $d$ letters $\Delta_{1}, \Delta_{2}, \ldots, \Delta_{d}$ (corresponding to the base triangles) as follows: $\theta^{(\mu)}\left(\Delta_{i}\right)=\Delta_{j}$ if $M_{i j}^{(\mu)}=1$ and $i \neq j$. In the other cases, $\Delta_{i}$ is mapped onto itself. Then clearly, $\left\langle\theta^{(\mu)} \| \mu \in\{1,2,3\}\right\rangle$ is a transitive permutation group on $X$, which we call the operator group of $D$. 
Suppose that $\left(D_{1}, D_{2}\right)$ is a pair of non-congruent planar isospectral domains constructed from unfolding an $r$-gon, $r \geq 3, d<\infty$ times. Since the $D_{i}$ are constructed by unfolding an $r$-gon, we can associate $r$ involutions $\theta_{i}^{(j)}$ to $D_{i}, j=1,2, \ldots, r$ and $i=1,2$. Define the operator groups

$$
G_{i}=\left\langle\theta_{i}^{(j)}\right\rangle
$$

Now suppose that

$$
G_{1} \cong \operatorname{PSL}(n, q) \cong G_{2},
$$

with $q$ a prime power and $n \geq 2$ a natural number.

The natural geometry on which $\operatorname{PSL}(n, q)$ acts (faithfully) is the $(n-1)$-dimensional projective space $\mathbf{P G}(n-1, q)$ over the finite field $\mathbb{F}_{q}$. It should be mentioned that $\operatorname{PSL}(n, q)$ acts transitively on the points of $\mathbf{P G}(n-1, q)$. So we can see the involutions $\theta_{i}^{(j)}$ for fixed $i \in\{1,2\}$ as automorphisms of $\mathbf{P G}(n-1, q)$ that generate $\operatorname{PSL}(n, q)$.

This means that for fixed $i \in\{1,2\}$ the triple

$$
\left(\mathbf{P G}(n-1, q),\left\{\theta_{i}^{(j)}\right\}, r\right)
$$

yields generalized projective isospectral data.

Theorem VII. 6 implies that $(n, q)$ is contained in $\{(3,2),(3,3),(4,2),(3,4)\}$ if $n \geq 3$.

Now suppose that $n=2$. We have to solve the equation

$$
r|\mathbf{P G}(1, q)|-\sum_{j=1}^{r} \operatorname{Fix}\left(\theta_{i}^{(j)}\right)=2(|\mathbf{P G}(1, q)|-1),
$$

for fixed $i \in\{1,2\}$, where $\operatorname{Fix}\left(\theta_{i}^{(j)}\right)$ is the number of fixed points in $\mathbf{P G}(1, q)$ of $\theta_{i}^{(j)}$. Since $|\mathbf{P G}(1, q)|=q+1$ and since a nontrivial element of $\mathbf{P S L}(2, q)$ fixes at most 2 points of $\mathbf{P G}(1, q)$, an easy calculation leads to a contradiction if $q \geq 3$.

Now let $q=2$. Then $\operatorname{PSL}(2,2)$ contains precisely 3 involutions, and they each fix precisely one point of $\mathbf{P G}(1,2)$. A numerical contradiction follows. 


\section{RELATED QUESTIONS}

We now consider some questions related to the main one elaborated on in the present paper.

\section{A. Boundary conditions}

So far we have mainly concentrated on billiards with Dirichlet boundary conditions. More recently attention has been concentrated on mixed Dirichlet-Neumann boundary conditions, that is, having either $\Psi=0$ or $\partial_{n} \Psi=0$ on different intervals of the boundary ( $n$ being the normal to the boundary). The answer to this problem is much simpler than the Dirichlet problem. Very simple instances of mixed-boundary condition isospectral pairs are proposed in (Levitin et al., 2006) (see also (Jakobson et al., 2006)). Their simplest example is reproduced at Fig. 15. Eigenfunctions are given by

$$
\sin \frac{\pi(m+1 / 2) x}{d} \sin \frac{\pi n y}{d}, \quad n \geq 1, m \geq 0
$$

for the square of size $d$, and

$$
\sin \frac{\pi(m+1 / 2) x}{d \sqrt{2}} \sin \frac{\pi(n+1 / 2) y}{d \sqrt{2}}-\sin \frac{\pi(n+1 / 2) x}{d \sqrt{2}} \sin \frac{\pi(m+1 / 2) y}{d \sqrt{2}}, \quad m>n \geq 0
$$

for the triangle of size $d \sqrt{2}$. These examples can be generalized: (Levitin et al., 2006) gives
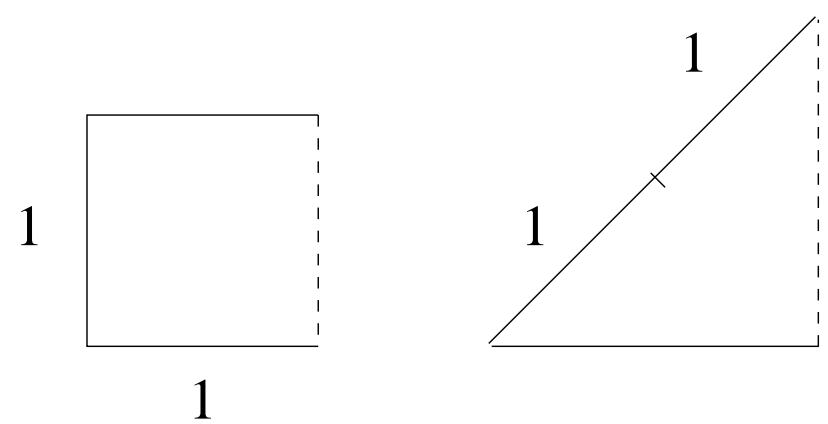

Figure 15 Isospectral billiards with mixed Neumann-Dirichlet boundary conditions. Solid line $=$ Dirichlet; dashed line $=$ Neumann.

a procedure to construct similar pairs. The idea is to construct an elementary domain, or 
"construction block", whose boundary is made of two line segments $a$ and $b$ on the plane, whose ends are joined by two arbitrary curves. Imposing any mixed Neumann-Dirichlet boundary conditions on the construction block boundary, one obtains a Neumann-Dirichlet isospectral pair by gluing the construction block together with its reflexion with respect to either $a$ or $b$ (and imposing Neumann b.c. to the segment itself, Dirichlet to its image). This technique can be further generalized by gluing together more copies of the construction block, yielding more complicated examples. In particular this method shows that for mixed boundary conditions it is possible to construct

- isospectral pairs such that one member is connected and the other is not;

- isospectral pairs such that one member is smooth and the other is not;

- isospectral 4-uples;

- billiards whose spectrum remains invariant when Dirichlet and Neumann boundaries are swapped. These billiards were investigated in (Jakobson et al., 2006). The simplest example is a billiard of semi-circular shape: if the equation of the billiard on the complex plane is given by $\{z \in \mathbb{C} ; 0 \leq \arg (z) \leq \pi ;|r| \leq 1\}$, the Dirichlet boundary conditions correspond to $\{z \in \mathbb{C} ;|r|=1, \pi / 4 \leq \arg (z) \leq 3 \pi / 4, \Re z<0\}$. A necessary condition for this Dirichlet-Neumann isospectrality is that the Dirichlet boundary has the same total length as the Neumann boundary.

Such domains have been investigated numerically (Driscoll and Gottlieb, 2003) as well as analytically (Okada and Shudo, 2001a), and experimental setups have been proposed in (Driscoll and Gottlieb, 2003).

All these examples have the property that the length difference between the Dirichlet boundary and the Neumann boundary are the same. This turns out Necessary conditions similar to those obtained from Weyl's law (77) applying to isospectral billiards derived in (Levitin et al., 2006) for mixed-b.c. isospectral billiards. In particular such isospectral pairs need to have same area, same length difference between the Dirichlet boundary and the Neumann boundary and same curvature-singularity properties, namely

$$
2 \int_{\partial B} \kappa(s) d s+\sum_{D D} \frac{\pi^{2}-\beta^{2}}{\beta}+\sum_{N N} \frac{\pi^{2}-\beta^{2}}{\beta}-\frac{1}{2} \sum_{D N} \frac{\pi^{2}+2 \beta^{2}}{\beta},
$$


where $\kappa$ is the curvature and $\beta$ represents the angles at the Dirichlet-Dirichlet, NeumannNeumann or Dirichlet-Neumann boundary intersetions.

Finally, we observe that for some of the examples produced in (Levitin et al., 2006), it was shown that two isospectral domains produce a different number of nodal domains (domains separated by nodal lines where $\Psi=0$, see section V.H).

\section{B. Isospectrality versus iso-length spectrality}

\section{Okada and Shudo's result on iso-length spectrality}

Let $D$ be a planar domain obtained by unfolding $N$ times the same triangular building block $B$ with sides $1,2,3$. Then the length spectrum is the set of lengths of closed trajectories (periodic orbits) of $D$. Any periodic orbit on $D$ can be regarded as a "lift" of a closed trajectory on $B$, because its projection is always a periodic orbit on $B$. (The converse is, of course, not necessarily true.) One observes that the number of closed lifts of a given closed trajectory on $B$ is counted as

$$
n^{D}(\gamma)=\operatorname{Tr}\left(M^{\left(\gamma_{m}\right)} M^{\left(\gamma_{m-1}\right)} \ldots M^{\left(\gamma_{1}\right)}\right)
$$

where $\gamma=\prod \gamma_{i}\left(\gamma_{i} \in\{1,2,3\}\right)$ denotes the sequence representing the order in which a given closed trajectory on $B$ hits the boundary segments. (The $M^{\left(\gamma_{j}\right)}$ 's are adjacency matrices.) Note that such a sequence is not uniquely determined by a given closed orbit - the number of closed lifts, however, is. So the length spectrum of $D$ is determined by the length spectrum of $B$ and by $n^{D}(\gamma)$. Hence, if one considers two domains $D$ and $D^{\prime}$ that are constructed by unfolding the same building block as above, it is sufficient to prove that $n^{D}(\gamma)=n^{D^{\prime}}(\gamma)$ for all possible sequences $\gamma$ in order to conclude "iso-length spectrality".

The following is now obvious.

Theorem VIII.1 (Y. Okada and A. Shudo (Okada and Shudo, 2001b)) Let D and $D^{\prime}$ be two unfolded domains obtained by $N$ times succesive reflections of the same building block. If $D$ and $D^{\prime}$ are transplantable, then $n^{D}(\gamma)=n^{D^{\prime}}(\gamma)$ for any sequence $\gamma$, so $D$ and $D^{\prime}$ are 
iso-length spectral.

Theorem VIII.2 (Y. Okada and A. Shudo (Okada and Shudo, 2001b)) Let $D$ and $D^{\prime}$ be two unfolded domains obtained by $N$ times succesive reflections of the same building block. If $n^{D}(\gamma)=n^{D^{\prime}}(\gamma)$ for any sequence $\gamma$, then $D$ and $D^{\prime}$ are transplantable, so also isospectral.

Proof. Let $G$ and $G^{\prime}$ - respectively corresponding to $D$ and $D^{\prime}$ — be the groups generated by the adjacency matrices:

$$
G=\left\langle M^{(\mu)}\right\rangle, \quad G^{\prime}=\left\langle N^{(\mu)}\right\rangle ;
$$

then clearly $G$ and $G^{\prime}$ are subgroups of the symmetric group $\mathbf{S}_{N}$ on $N$ letters. Let $\mathbf{F}_{3}$ be a free group generated by symbols $a, b$ and $c$. Define the surjective homomorphism

$$
\Phi_{D}: \mathbf{F}_{3} \mapsto G: \gamma=\gamma_{1} \gamma_{2} \ldots \gamma_{m} \mapsto M^{\left(\gamma_{m}\right)} M^{\left(\gamma_{m-1}\right)} \ldots M^{\left(\gamma_{1}\right)}
$$

Then

$$
G \cong \mathbf{F}_{3} / \operatorname{ker} \Phi_{D} \text { and } G^{\prime} \cong \mathbf{F}_{3} / \operatorname{ker} \Phi_{D^{\prime}}
$$

the latter notation being obvious.

Now assume that $n^{D}(\gamma)=n^{D^{\prime}}(\gamma)$ for any sequence $\gamma$. Then

$$
\begin{gathered}
\operatorname{ker} \Phi_{D}=\left\{\gamma \| \Phi_{D}(\gamma)=\mathbb{I}\right\}=\left\{\gamma \| n_{D}(\gamma)=N\right\}=\left\{\gamma \| n_{D^{\prime}}(\gamma)=N\right\} \\
=\left\{\gamma \| \Phi_{D^{\prime}}(\gamma)=\mathbb{I}\right\}=\operatorname{ker} \Phi^{D^{\prime}} .
\end{gathered}
$$

So the map

$$
\Delta: G \mapsto G^{\prime}: \Phi^{D}(\gamma) \mapsto \Phi^{D^{\prime}}(\gamma)
$$

yields an isomorphism between $G$ and $G^{\prime}$.

Let (identity maps) 


$$
\rho^{D}: G \mapsto \mathbf{G L}(N, \mathbb{C}), \quad \rho^{D^{\prime}}: G^{\prime} \mapsto \mathbf{G L}(N, \mathbb{C})
$$

be linear representations of $G$ and $G^{\prime}$, respectively. Since the latter groups are isomorphic,

$$
\rho=\rho^{D^{\prime}} \circ \Delta: \Phi^{D}(\gamma) \mapsto \Phi^{D^{\prime}}(\gamma) \in \mathbf{G L}(N, \mathbb{C})
$$

is another linear representation of $G$. Since $n^{D}(\gamma)$ and $n^{D^{\prime}}(\gamma)$ become (equal) characters of the representations $\rho^{D}$ and $\rho$ respectively, the representations are similar. So there exists an invertible matrix $T$ for which

$$
T M^{(\mu)}=N^{(\mu)} T
$$

for any $\mu$. So $D$ and $D^{\prime}$ are transplantable.

\section{Penrose-Lifshits mushrooms}

Michael Lifshits (unpublished), exploiting a type of construction attributed to R. Penrose (see, e.g., (Rauch, 1978)), constructed a class of pairs of planar domains that, while not isometric, have periodic geodesics of exactly the same lengths (including multiplicities). At least when the boundaries are smooth $\left(C^{\infty}\right)$, it follows that the two billiards have the same wave invariants, in the sense that the traces of their wave groups, $\cos (t \sqrt{\Delta})$, differ at most by a smooth function (Melrose, 1996). In a recent review of the inverse spectral problem (Zelditch, 2004) S. Zelditch asked whether the Dirichlet Laplacians, $\Delta$, for the two domains are necessarily isospectral (judging that proposition "dubious" but not yet refuted). Given the refutation, such billiards provide a kind of converse to the famous examples of drums that sound the same, being drums that sound different but are very similar geometrically - in fact, in the geometrical features deemed most relevant to the spectrum.

In this section we describe a construction of smooth Penrose-Lifshits mushroom pairs that are not isospectral, following (Fulling and Kuchment, 2005). Since the domains are smooth (but not convex), the spectral difference is not attributable to diffraction from 
corners, which would muddy the definition or the relevance of "periodic geodesics".

The construction of a mushroom starts from a half-ellipse $E$ with foci $F$ and $\tilde{F}$ :

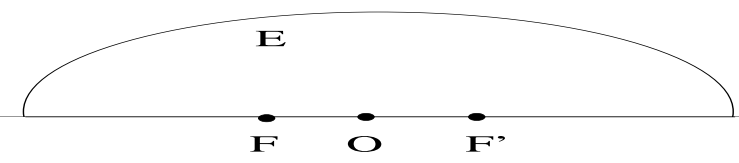

We use the tilde, whether applied to regions, curves, or points, to indicate the operation of reflection through the minor axis of the ellipse. If two entities are interchanged by that reflection, we call them dual. Next, add two "bumps", $B_{1}$ on the left and $B_{2}$ on the right, with $\tilde{B}_{1} \neq B_{2}$, to form a smooth domain $\Omega$ :

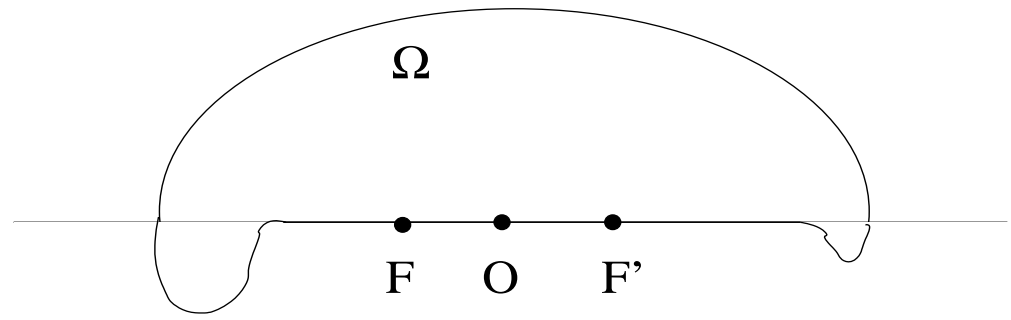

Finally, add another bump (not self-dual) between the foci in two dual ways ( $M$, respectively $\tilde{M}$ ) to get two domains $\Omega_{j}$ :

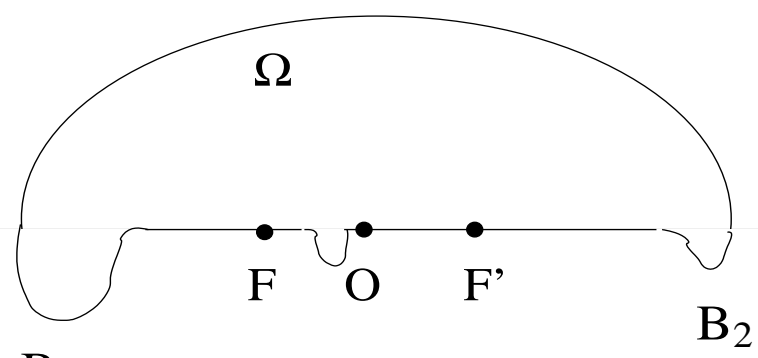

$\mathrm{B}_{1}$ 
respectively

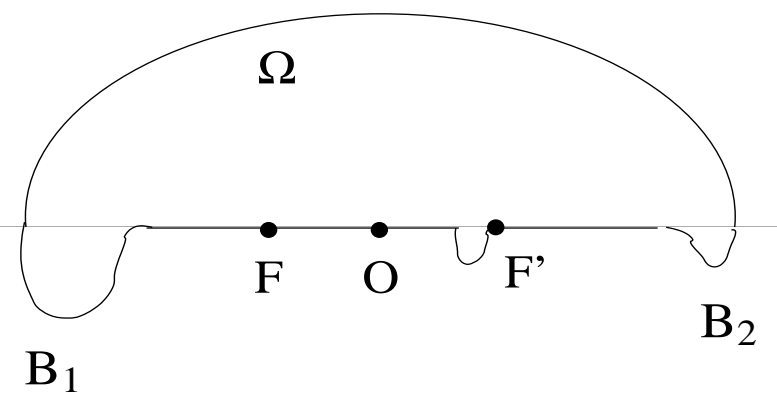

We call the domains $\Omega_{1}$ and $\Omega_{2}$ constructed in this manner a Penrose-Lifshits mushroom pair.

We repeat that the bumps can be added in such a way that the boundaries remain smooth. That assumption, however, is needed mainly to draw a clean conclusion about equality of the length spectra.

Theorem VIII.3 (S. A. Fuller and P. Kuchment (Fulling and Kuchment, 2005)) If $B_{1}$ and $B_{2}$ are given and not dual, then there exist dual bumps $M$ and $\tilde{M}$ such that the resulting Penrose-Lifshits mushrooms $\Omega_{j}$ have the same length spectra and wave invariants but are not isospectral.

Proof. First we review the proof that the length spectra coincide (Melrose, 1996; Zelditch, 2004). The geodesics in an ellipse fall into two disjoint categories (Berry, 1981; Keller and Rubinow, 1960; Rauch, 1978):

- those that intersect the major axis between the foci, and

- those that do so at or beyond the foci.

The only exception is the major axis itself. The smoothness assumption guarantees that the major axis will not bifurcate in $\Omega_{j}$ by diffraction.

It follows that a similar division holds for the domains $\Omega_{j}$ we have just described: any geodesic originating in a bump $B_{1}$ or $B_{2}$ can never reach a bump $M$ or $\tilde{M}$, and vice versa. Now, the geodesics that do not intersect the focal segment $F \tilde{F}$ are exactly the same for the two domains. On the other hand, those for $\Omega_{1}$ that do intersect this segment are identified one-to-one with their duals in $\Omega_{2}$ by the reflection operation. 
This shows length isospectrality.

Now we have to show nonisospectrality for some choice of $M$. Consider the spectrum of $\Omega_{1}$ assuming that the bump $M$ is small and has support on the left half of the focal segment; i.e., to construct $\Omega_{1}$ the (open) segment $F O$ in the boundary of $\Omega$ is perturbed by the graph of a smooth, compactly supported (and nonpositive) function $\epsilon f(x)$, where $\epsilon$ is a small parameter. Let $\psi_{0}$ be the ground state of the Dirichlet Laplacian on $\Omega$ and $\lambda_{0}$ be the corresponding lowest eigenvalue. The known Rayleigh-Hadamard formula for change of the spectrum under domain perturbations (e.g., (Garabedian and Schiffer, 1952; L. Ivanov and Krein, 1977; Zelditch, 2004) or (Garabedian, 1998, Section 15.1, Exercise 9)) shows that if

$$
\int_{\partial \Omega}\left(\frac{\partial \psi_{0}(x)}{\partial \nu}\right)^{2} f(x) d \sigma(x) \neq 0,
$$

where $\frac{\partial \psi_{0}(x)}{\partial \nu}$ is the normal derivative of the eigenfunction on the boundary, then the lowest eigenvalue $\lambda_{0}$ changes under the perturbation. This integral gives the derivative at $\epsilon=0$ of the lowest eigenvalue with respect to $\epsilon$. Thus, if one could guarantee that the values of this integral are different for the two small perturbation domains $\Omega_{j}$, this would imply their non-isospectrality: the lowest Dirichlet eigenvalues would change with different velocities. Since the choice of the perturbation shape $f$ is in our hands, in order to make these integrals different, and thus domains nonisospectral, it is sufficient to have two mutually dual segments inside the focal segment such that the square of the normal derivative of the ground state is not an even function on their union, $I$. Indeed, in this case we could find an even perturbation $f$ that would provide nonequal integrals (in fact, almost any perturbation would do).

\section{Spectral problems for Lie geometries}

There exists a vast literature on spectral problems for (finite) graphs - see the excellent paper (van Dam and Haemers, 2003) on that behalf. In this section we want to consider a spectral - "Kac type" — problem for graphs which are associated to the most important incidence geometries. 
We have already seen that certain simple Lie groups turn up in the construction theory of counter examples. It seems natural to extend (variations of) Kac theory to the natural geometries on which such groups act as automorphism groups. Since the most important rank 2 geometries are the so-called "generalized polygons", we address the extension for these Lie geometries. Generalized polygons are the natural generalizations of the projective planes, but of course one could address the question(s) we consider below to other incidence geometries.

First we need to introduce these synthetic structures.

\section{Generalized polygons}

In this section we state the definition of the central Lie type geometries of rank 2, namely the "generalized polygons", and introduce some standard notation and notions. Generalized polygons were introduced by J. Tits in his celebrated paper (Tits, 1959), in order to have a geometric interpretation of certain Chevalley groups of rank 2. They are also the building bricks of (Tits) buildings, the natural geometries for the groups with a BN-pair. A group $G$ is said to have a $B N$-pair $(B, N)$, where $B, N$ are subgroups of $G$, if the following properties are satisfied:

(BN1) $\langle B, N\rangle=G$;

(BN2) $H=B \cap N \unlhd N$ and $N / H=W$ is a Coxeter group (see, e.g., (Tits, 1974)) with distinct generators $s_{1}, s_{2}, \ldots, s_{n}$;

(BN3) $B s_{i} B w B \subseteq B w B \cup B s_{i} w B$ whenever $w \in W$ and $i \in\{1,2, \ldots, n\}$;

(BN4) $s_{i} B s_{i} \neq B$ for all $i \in\{1,2, \ldots, n\}$.

The subgroup $B$, respectively $W$, is a Borel subgroup, respectively the Weyl group, of $G$. The natural number $n$ is called the rank of the BN-pair.

Example. Suppose $\mathbf{P G}(1, q)$ is the projective line over the finite field $\mathbb{F}_{q}$; so $\mathbf{P G}(1, q)$ has $q+1$ points. Consider the natural action of $\operatorname{PSL}(2, q)$ on $\mathbf{P G}(1, q)$, and let $x$ and $y$ be 
distinct points of the projective line. Put $B=\operatorname{PSL}(2, q)_{x}$ and $N=\operatorname{PSL}(2, q)_{\{x, y\}}$. Then $(B, N)$ is a BN-pair for $\operatorname{PSL}(2, q)$. Here $N /(B \cap N)=W$ is just the group of order 2.

Example. Consider the Desarguesian projective plane $\mathbf{P G}(2, q)$, and $\operatorname{PSL}(3, q)$ in its natural action on the latter plane. Let $(x, L)$ be an incident point-line pair, and $\Delta$ a triangle (in the ordinary sense) that contains $x$ as a point and $L$ as a side. Put $B=\operatorname{PSL}(3, q)_{(x, L)}$ and $N=\operatorname{PSL}(3, q)_{\Delta}$; then $(B, N)$ is a $\operatorname{BN}$-pair for $\operatorname{PSL}(3, q)$ and $N /(B \cap N)=W$ is the dihedral group of order 6 .

The reader may want to use the monographs (J. A. Thas and Maldeghem, 2006; Maldeghem, 1998; Payne and Thas, 1984; Thas, 2004) as standard references on the subject of generalized polygons.

\section{Definition and properties}

We start with a synthetic definition. Let $n \geq 3$ be a natural number. A (thick) generalized $n$-gon or (thick) generalized polygon (GP) is a point-line geometry $\Gamma=(\mathcal{P}, \mathcal{B}, \mathbf{I})$, where $\mathcal{P}$ is the point set, $\mathcal{B}$ the line set and $\mathbf{I} \subset(\mathcal{P} \times \mathcal{B}) \cup(\mathcal{B} \times \mathcal{P})$ the symmetric incidence relation, so that the following axioms are satisfied:

(i) $\Gamma$ contains no $k$-gon (in the ordinary sense), for $2 \leq k<n$;

(ii) any two elements $x, y \in \mathcal{P} \cup \mathcal{B}$ are contained in some ordinary $n$-gon in $\Gamma$;

(iii) there exists an ordinary $(n+1)$-gon in $\Gamma$.

The point graph of a point-line geometry is the graph of which the vertices are the points of the geometry, and for which two vertices are joined by an edge if they are collinear in the geometry. Equivalently, a generalized polygon could be defined as a point-line geometry for which the point graph is bipartite of diameter $n$ and girth $2 n$.

The generalized 3-gons are precisely the projective planes. If (iii) is not satisfied for $\Gamma$, then $\Gamma$ is called thin. Otherwise, it is called thick. Each thick generalized $n$-gon, $n \geq 3, \Gamma$ 


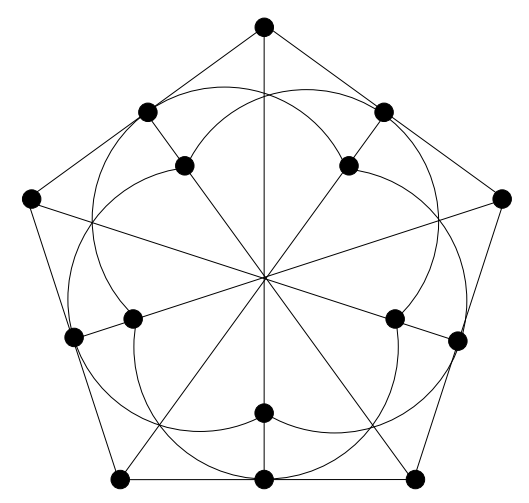

Figure 16 The unique generalized quadrangle of order 2 : the symplectic $\mathbf{W}(3,2)$.

has an order: there are (not necessarily finite) constants $s>1$ and $t>1$ so that each point is incident with $t+1$ lines and each line is incident with $s+1$ points. We then say that $\Gamma$ "has order $(s, t)$ ". Note that, for a point $x$ and a line $L, " x \mathbf{I} L$ " means that $(x, L) \in \mathbf{I}$ (and so also $(L, x) \in \mathbf{I})$.

In this paper we only consider thick GPs.

Now let $G$ be a group with a BN-pair $(B, N)$ of rank 2 .

One can associate a generalized polygon $\mathcal{B}(G)$ to the group $G$ in the following way. For this purpose, define $P_{1}=\left\langle B, B^{s_{1}}\right\rangle$ and $P_{2}=\left\langle B, B^{s_{2}}\right\rangle$.

- Call the right cosets of $P_{1}$ "points".

- Call the right cosets of $P_{2}$ "lines".

- Call two such (distinct) cosets "incident" if their intersection is nonempty (so $P_{1} g$ is incident with $P_{2} h, g, h \in G$, if $P_{1} g \cap P_{2} h \neq \emptyset$ ).

Then $\mathcal{B}(G)$ is a GP - say a generalized $n$-gon for some natural number $n$ - on which $G$ acts naturally as an automorphism group that permutes transitively the ordered $n$-gons (in the ordinary sense).

Conjecture [J. Tits, (Tits, 1974, §11.5.1)]. If a finite thick generalized $n$-gon is such that the automorphism group permutes transitively the ordered n-gons (that is, if $\Delta$ is associated with a BN-pair), then $\Delta$ is isomorphic with the GP of an absolutely simple group over a finite field, or with the GP of a Ree group of Type ${ }^{2} \mathbf{F}_{4}$ over a finite field. 
For more on the classification of BN-pairs of rank 2, see (Thas, a,c,d; Thas and Maldeghem).

\section{Duality principle}

Let $\Gamma=(\mathcal{P}, \mathcal{B}, \mathbf{I})$ be a GP of order $(s, t)$. Then $\Gamma^{D}=(\mathcal{B}, \mathcal{P}, \mathbf{I})$ clearly again is a GP, but now of order $(t, s)$. (The latter geometry is called the point-line dual of $\Gamma$.) So any theorem which holds for a GP, has a dual interpretation; we call this principle "Duality Principle".

\section{Automorphisms and isomorphisms}

Let $\Gamma=(\mathcal{P}, \mathcal{B}, \mathbf{I})$ and $\Gamma^{\prime}=\left(\mathcal{P}^{\prime}, \mathcal{B}^{\prime}, \mathbf{I}^{\prime}\right)$ be GPs. Then an isomorphism between $\Gamma$ and $\Gamma^{\prime}$ is a pair $(\alpha, \beta)$ for which $\alpha$ is a bijection between $\mathcal{P}$ and $\mathcal{P}^{\prime}, \beta$ is a bijection between $\mathcal{B}$ and $\mathcal{B}^{\prime}$, and $x \mathbf{I} L$ (in $\Gamma$ ) if and only if $x^{\alpha} \mathbf{I} L^{\beta}$. If there is an isomorphism between $\Gamma$ and $\Gamma^{\prime}$, we say they are "isomorphic", and write $\Gamma \cong \Gamma^{\prime}$.

If $\Gamma=\Gamma^{\prime}$ one speaks of an "automorphism". The set of all automorphisms of a GP forms a group, and the classical examples of GPs are those examples that are associated to a Chevalley group (or, equivalently, to a "classical" BN-pair), cf. (Maldeghem, 1998) for more details.

\section{Collinearity matrices}

Suppose $\Gamma=(\mathcal{P}, \mathcal{B}, \mathbf{I})$ is a finite $\mathrm{GP}$ ( $\Gamma$ has a finite number of points and lines) of order $(s, t)$, put $|\mathcal{P}|=v$, and let $\left\{x_{1}, x_{2}, \ldots, x_{v}\right\}=\mathcal{P}$ be the point set. Define the collinearity matrix $\mathbf{A}(\Gamma)=\mathbf{A}$ as the $v \times v$-matrix $\left(a_{i j}\right)$ for which $a_{i j}=1$ if $x_{i} \sim x_{j} \neq x_{i}$ (the latter notation meaning that $x_{i}$ and $x_{j}$ are different collinear points), and 0 otherwise. So it is the adjacency matrix of the point graph of $\Gamma$. The (point) spectrum of $\Gamma$ is the spectrum of $\mathbf{A}$, and we denote it by $\operatorname{spec}(\mathbf{A})$. 
The following quantum mechanical question is the Kac inverse problem for the theory of GPs.

QUESTION VIII.4 Let $\Gamma$ and $\Gamma^{\prime}$ be distinct finite thick generalized polygons with associated collinearity matrices $\mathbf{A}$ and $\mathbf{A}^{\prime}$, respectively. Does $\operatorname{spec}(\mathbf{A})=\operatorname{spec}\left(\mathbf{A}^{\prime}\right)$ imply that $\Gamma \cong \Gamma^{\prime}$ ?

Clearly, a similar problem can be posed for the line spectrum, but as points and lines play essentially the same role in a GP, we will only consider the question in its above form.

We will show that Question VIII.4 can be reduced to a very important question in the theory of GPs.

Theorem VIII.5 (K. Thas (Thas, b)) Let $\Gamma$ and $\Gamma^{\prime}$ be distinct finite thick generalized polygons with associated collinearity matrices $\mathbf{A}$ and $\mathbf{A}^{\prime}$, respectively. Then $\operatorname{spec}(\mathbf{A})=\operatorname{spec}\left(\mathbf{A}^{\prime}\right)$ if and only if $\Gamma$ and $\Gamma^{\prime}$ have the same order.

The next section contains the proof of Theorem VIII.5.

\section{E. Point spectra and order}

Let $\Gamma$ be a finite thick GP of order $(s, t)$, with associated collinearity matrix A. Our first concern is to calculate $\operatorname{spec}(\mathbf{A})$.

First of all, we recall a celebrated theorem of Feit and Higman (Feit and Higman, 1964):

A finite thick generalized $n$-gon exists if and only if $n \in\{3,4,6,8\}$.

We will do a case-by-case analysis according to this result.

Case $n=3$

Recall that a generalized 3-gon is the same as an axiomatic projective plane.

Now let $\Gamma$ be a finite projective plane of order $n, n \geq 2$, and put $n^{2}+n+1=\mathfrak{v}$, its number of points. Then $\mathbf{A}=\mathbf{J}_{\mathfrak{v}}-\mathbf{I}_{\mathfrak{v}}$, where $\mathbf{J}_{\mathfrak{v}}$ is the all $1 \mathfrak{v} \times \mathfrak{v}$-matrix, and $\mathbf{I}_{\mathfrak{v}}$ the $\mathfrak{v} \times \mathfrak{v}$-identity matrix. It follows that 


$$
\operatorname{spec}(\mathbf{A})=\{-1, \mathfrak{v}-1\}
$$

So if the spectra of two finite projective planes coincide, their orders also do.

Case $n=4$

Let $\Gamma$ be a thick generalized 4-gon, or also "GQ" ("generalized quadrangle") of order $(s, t)$. Then by (Payne and Thas, 1984, 1.2.2), we have

$$
\operatorname{spec}(\mathbf{A})=\{-t-1, s-1, s(t+1)\}
$$

Now let $\Gamma^{\prime}$ also be a thick GQ, with the same spectrum, of order $\left(s^{\prime}, t^{\prime}\right)$. There is only one negative eigenvalue, so $-t-1=-t^{\prime}-1$ and $t=t^{\prime}$. Since $s-1<s(t+1)\left(s^{\prime}-1<s^{\prime}\left(t^{\prime}+1\right)\right)$, it also follows that $s=s^{\prime}$, and hence $\Gamma$ and $\Gamma^{\prime}$ have the same order.

Case $n=6$

For this case, we need one more definition. A distance regular graph $G$ with diameter $d$ is a regular connected graph with valency $k$ for which there exist natural numbers $b_{0}=$ $k, b_{1}, \ldots, b_{d-1} ; c_{1}=1, c_{2}, \ldots, c_{d}$ such that for each pair of vertices $x$ and $y$ at distance $j$, we have

- $\left|\Gamma_{j-1}(y) \cap \Gamma_{1}(x)\right|=c_{j}, 1 \leq j \leq d ;$

- $\left|\Gamma_{j+1}(y) \cap \Gamma_{1}(x)\right|=b_{j}, 0 \leq j \leq d-1$.

Now define the constants $a_{j}=\left|\Gamma_{j}(y) \cap \Gamma_{1}(x)\right|$ for $0 \leq j \leq d$. A result of (A. E. Brouwer and Neumaier, 1989) claims that the eigenvalues of the point graph of $G$ are the eigenvalues of the following "intersection matrix": 


$$
\left(\begin{array}{cccccc}
0 & 1 & & & & \\
k & a_{1} & c_{2} & & & \\
& b_{1} & a_{2} & & & \\
& & b_{2} & \ldots & & \\
& & & \cdots & \cdot & \\
& & & & \cdot & \\
& & & & c_{d} & \\
& & & & b_{d-1} & a_{d}
\end{array}\right) .
$$

It just so happens to be that the collinearity graph of a generalized hexagon $(=\mathrm{a}$ generalized 6-gon) is distance regular with diameter 3. An easy exercise yields, for a thick generalized hexagon of order $(s, t)$, the following intersection matrix:

$$
\mathbf{B}=\left(\begin{array}{cccc}
0 & 1 & 0 & 0 \\
s(t+1) & s-1 & 1 & 0 \\
0 & s t & s-1 & t+1 \\
0 & 0 & s t & (t+1)(s-1)
\end{array}\right)
$$

The determinant of $\mathbf{B}-x \mathbf{I}_{4}$ has the following roots:

$$
x=-t-1, \quad x=s(t+1), \quad x=s-1-\sqrt{s t}, \quad x=s-1+\sqrt{s t} .
$$

One observes that $-t-1$ is strictly the smallest eigenvalue, while $s(t+1)$ is the largest. It now easily follows that if a generalized hexagon of order $\left(s^{\prime}, t^{\prime}\right)$ has the same spectrum as $\Gamma$, then it has the same order. 
Case $n=8$

Let $\Gamma$ be a thick generalized octagon (8-gon) of order $(s, t)$. Again, the point graph is distance regular, now with diameter 4 . The intersection matrix is easily seen to be the following:

$$
\mathbf{B}=\left(\begin{array}{ccccc}
0 & 1 & 0 & 0 & 0 \\
s(t+1) & s-1 & 1 & 0 & 0 \\
0 & s t & s-1 & 1 & 0 \\
0 & 0 & s t & s-1 & t+1 \\
0 & 0 & 0 & s t & (t+1)(s-1)
\end{array}\right)
$$

which has eigenvalues

$$
x=-t-1, \quad x=s-1, \quad x=s(t+1), \quad x=s-1-\sqrt{2 s t}, \quad x=s-1+\sqrt{2 s t} .
$$

The third largest eigenvalue is $s-1$, so, if $\Gamma^{\prime}$ is a thick generalized octagon of order $\left(s^{\prime}, t^{\prime}\right)$ with the same spectrum, then $s=s^{\prime}$. As $s(t+1)$ is the largest eigenvalue of $\operatorname{spec}(\mathbf{A})$, it follows that $t=t^{\prime}$.

This ends the proof of Theorem VIII.5.

\section{F. Concluding remarks}

In this section, we make some comments on generalized polygons that are characterized by their order.

Projective Planes. For some small values, e.g. $n=2$, it is known that there is a unique projective plane of order $n$ (up to isomorphism). It is well-known, however, that as soon 
as $n$ is large enough and not a prime, nonisomorphic examples exist. On the other hand, for $p$ a prime, only one example is known, namely the classical example $\mathbf{P G}(2, p)$ arising from a BN-pair in $\operatorname{PSL}(2, p)$.

GENERALIZED QUADRANGLES. Many infinite classes of GQs are known, and several examples with small parameters are completely determined by their order. We refer to (Payne and Thas, 1984, Chapter 6) for these examples. We make some comments according to the known orders. Below, $q$ is always a prime power. We also assume $s \leq t$ by reasons of duality. (Details and references can be found in (Thas, 2004, Chapter 3).)

- $(s, t)=\left(q^{2}, q^{3}\right)$. Only one example is known (for each $q$ ), namely the Hermitian quadrangle $\mathbf{H}\left(4, q^{2}\right)$.

- $(s, t)=(q-1, q+1)$. If $q \geq 8$ and $q$ is even, nonisomorphic examples are known for every $q$. In the other cases, only unique examples are known.

- $(s, t)=(q, q)$. If $q$ is odd, nonisomorphic examples are known for every $q$. If $q \geq 8$ and $q$ is even, we have the same remark. The other values give unique examples.

- $(s, t)=\left(q, q^{2}\right)$. If $q \geq 5$, nonisomorphic examples are known for every $q$. The examples of order $(2,4)$ and $(3,9)$ are unique.

GENERALIZED HEXAGONS. Up to duality, only two classes of generalized hexagons are known (both associated to classical groups): the split Cayley hexagons $\mathbf{H}(q)$ of order $q, q$ a prime power, and the twisted triality hexagons $\mathbf{T}\left(q, q^{3}\right)$ of order $\left(q, q^{3}\right)$, cf. (Maldeghem, 1998, Chapter 2). We know that

$$
\mathbf{H}(q) \cong \mathbf{H}(q)^{D} \text { if and only if } q \text { is a power of } 3 \text { (Maldeghem, 1998). }
$$

So if $q$ is not as such, $\mathbf{H}(q) \approx \mathbf{H}(q)^{D}$, while both have the same spectrum.

GENERALIZED OCTAGONS. Up to duality, the only known thick finite generalized octagons are the Ree-Tits octagons $\mathbf{O}(q)$, where $q$ is an odd power of 2, and can be constructed from a BN-pair in the Ree groups of Type ${ }^{2} \mathbf{F}_{4}$ (Maldeghem, 1998, Chapter 2). They have order $\left(q, q^{2}\right)$. 
Finally, the reader is referred to the monograph (D. M. Cvetkovic and Sachs, 1998) for more information on graph spectra. Also, the recent paper (van Dam and Haemers, 2003) surveys the known cases of graphs which are determined by their spectrum. In that paper, also some generalized quadrangles with small parameters are mentioned which are uniquely determined by their spectrum. Since such examples must have the property that they are determined by their order, the reference (Payne and Thas, 1984, Chapter 6) also yields these examples. 


\section{G. Homophonic pairs}

Homophonic pairs in $\mathbb{R}^{2}$ are nonisometric compact domains which are not isospectral, but which have a distinguished point such that the corresponding (normalized) Dirichlet eigenfunctions take equal values at these points. This could be interpreted in the following way: if the corresponding drums are struck at these special points, then they sound the same in such a way that every frequency will be excited to the same intensity for each. An example of two billiards which are not isospectral but homophonic (Buser et al., 1994) is provided in the gallery of examples (example $21_{1}$ right). These billiards sound the same when struck at the interior points where six triangles meet.

\section{OPEN PROBLEMS}

Perhaps the single most important open problem in Kac theory is the following:

Show that for all $N \in \mathbb{N}$ there exists an $N^{*} \geq N$ such that there are isospectral pairs on $N^{*}$ tiles.

(Equivalently, show that there are infinitely many pairs of involution graphs which yield isospectral pairs.)

So far, only for $N \leq 21$ values of $N$ are known for which this question has a positive answer.

Further interesting problems in construction theory are:

Construct isospectral pairs which are not transplantable.

Derive criterions for pairs of involution graphs to yield isospectral plane domains.

Construct isospectral pairs on $\infty$ tiles (by a free construction?).

Construct isospectral $\mathbb{R}^{2}$-domains with smooth boundaries (independent of the tiling method.) 
Find examples of (planar) isospectral pairs not coming from Sunada triples, or still arising from Sunada triples but not being transplantable.

On the group theoretical level, we pose the following question.

Are the operator groups of (transplantable) isospectral pairs always 2-transitive? If so, the classification of finite simple groups could be used to classify such operator groups. In the same spirit, one could ask as to whether other finite simple groups can act as operator groups.

Develop a theory of isospectral "domains" for (= on) general buildings. Note that the projective completion of $\mathbb{R}^{2}$ is a rank 2-building over $\mathbb{R}$.

The same questions could all be formulated for "isospectral $n$-tuples", $n>2$. 


\section{Acknowledgments}

The second author is a Postdoctoral Fellow of the Fund for Scientific Research — Flanders (Belgium).

This paper was partly written while the second author was visiting the Discrete Mathematics group of the Technical University of Eindhoven, The Netherlands, whose hospitality he gratefully acknowledges.

The paper was finished while both authors were hosted by Institut Henri Poincaré (IHP) at Paris, whose hospitality is gratefully acknowledged. 
Appendix A: The finite simple groups

In this appendix we list the finite simple groups.

Notation. $n$ is a positive integer, $q>1$ is a power of a prime number $p$, and is the order of some underlying finite field. The groups we list may not be simple; often one has to factor out the central subgroup to obtain the simple group, compare (Gordon, 1986, p. 490-491).

\section{Infinite Families.}

- Cyclic groups: $\mathbb{Z}_{p}$

- $\mathbf{A}_{n}, n>4$ : Alternating groups

- $\mathbf{A}_{n}(q)$ : Chevalley groups, linear groups; note that $A_{n}(q) / Z\left(A_{n}(q)\right) \cong \mathbf{P S L}(n+1, q)$

- $\mathbf{B}_{n}(q), n>1$ : Chevalley groups, orthogonal group

- $\mathbf{C}_{n}(q), n>2$ : Chevalley groups, symplectic groups

- $\mathbf{D}_{n}(q), n>3$ : Chevalley groups, orthogonal groups

- $\mathbf{E}_{6}(q)$ : Chevalley groups

- $\mathbf{E}_{7}(q)$ : Chevalley groups

- $\mathbf{E}_{8}(q)$ : Chevalley groups

- $\mathbf{F}_{4}(q)$ : Chevalley groups

- $\mathbf{G}_{2}(q)$ : Chevalley groups

- ${ }^{2} \mathbf{A}_{n}\left(q^{2}\right), n>1$ : Steinberg groups, unitary groups

- ${ }^{2} \mathbf{D}_{n}\left(q^{2}\right), n>3$ : Steinberg groups, orthogonal groups

- ${ }^{2} \mathbf{E}_{6}\left(q^{2}\right)$ : Steinberg groups

- ${ }^{3} \mathbf{D}_{4}\left(q^{3}\right)$ : Steinberg groups 
- ${ }^{2} \mathbf{B}_{2}\left(2^{2 n+1}\right)$ : Suzuki groups

- ${ }^{2} \mathbf{F}_{4}\left(2^{2 n+1}\right)$ : Ree groups, Tits group

- ${ }^{2} \mathbf{G}_{2}\left(3^{2 n+1}\right)$ : Ree groups

Sporadic Groups.

(1) Mathieu group $\mathbf{M}_{11}$

(2) Mathieu group $\mathbf{M}_{12}$

(3) Mathieu group $\mathbf{M}_{22}$

(4) Mathieu group $\mathbf{M}_{23}$

(5) Mathieu group $\mathbf{M}_{24}$

(6) Janko group $\mathbf{J}_{1}$

(7) Janko group $\mathbf{J}_{2}$

(8) Janko group $\mathbf{J}_{3}$

(9) Janko group $\mathbf{J}_{4}$

(10) Conway group $\mathrm{Co}_{1}$

(11) Conway group $\mathrm{Co}_{2}$

(12) Conway group $\mathrm{Co}_{3}$

(13) Fischer group $\mathbf{F i}_{22}$

(14) Fischer group $\mathbf{F i}_{23}$

(15) Fischer group $\mathbf{F i}_{24}$

(16) Higman-Sims group HS

(17) McLaughlin group McL

(18) Held group He 
(19) Rudvalis group $\mathrm{Ru}$

(20) Suzuki sporadic group Suz

(21) O’Nan group $\mathrm{O}^{\prime} \mathbf{N}$

(22) Harada-Norton group $\mathbf{H N}$

(23) Lyons group Ly

(24) Thompson group Th

(25) Baby Monster group B

(26) Fischer-Griess Monster group M

Appendix B: Gallery of examples

1. Some modes
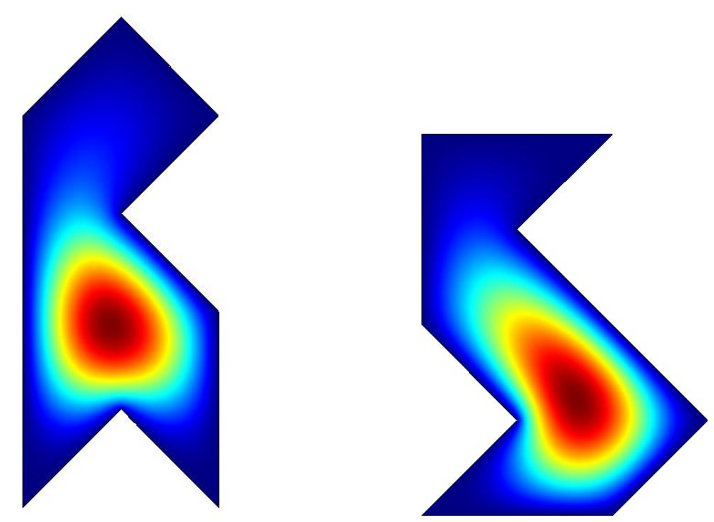

Figure 17 Fundamental modes

2. The 17 families of isospectral pairs and their mathematical properties

The following gallery presents the 17 known families of isospectral pairs, as obtained in (Buser et al., 1994; Giraud, 2005b; Okada and Shudo, 2001a). All are based on a Sunada triple $\left(G, G_{1}, G_{2}\right)$, where $G=\operatorname{PSL}(n+1, q)$ is the special linear automorphism group 

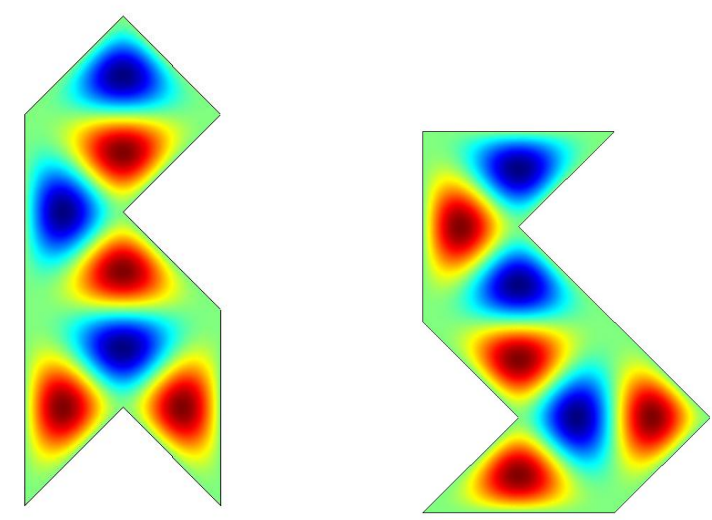

Figure 18 Triangular modes
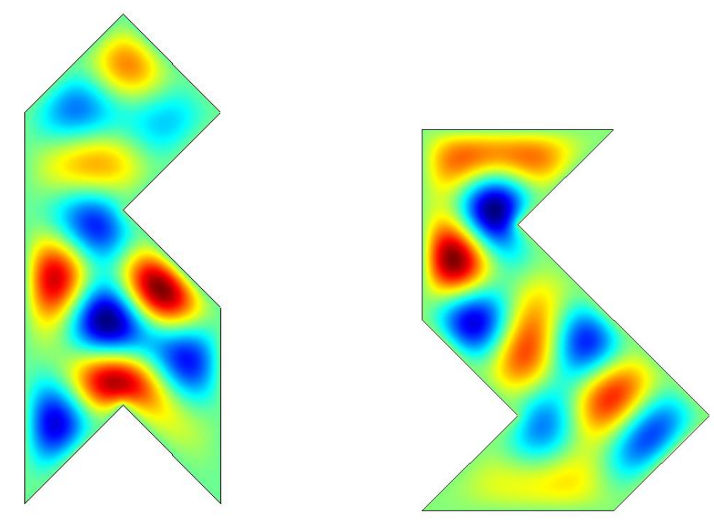

Figure 19 15-th mode

of a finite projective space of $\left(q^{n+1}-1\right) /(q-1)$ points, and $G_{1}, G_{2}$ are two subgroups respectively generated by $a_{1}, b_{1}, c_{1}$ and $a_{2}, b_{2}, c_{2}$ given below. These automorphisms are collineations of order 2 of the underlying finite projective space; $a_{1}, b_{1}, c_{1}$ act on points while $a_{2}, b_{2}, c_{2}$ act on lines, numbered from 0 to $\left(q^{n+1}-1\right) /(q-1)-1$.

Interestingly, the structure of pairs $13_{6}$ and $15_{2}$ forbids to construct any proper billiard, that is structures where triangles do not overlap. It is quite simple to convince oneself of this fact. In the case of the billiard $13_{6}$ (see Fig. 28), the initial triangle is unfolded six times around each of its corner. Clearly, to have a non-overlapping billiard, each angle should be less than $\pi / 3$, which is impossible unless the initial triangle is equilateral.

For the billiard $15_{2}$ (see Fig. 33), the initial triangle is unfolded six times around two of its corners, four times around the third one, thus two angles have to be less than $\pi / 3$ and 

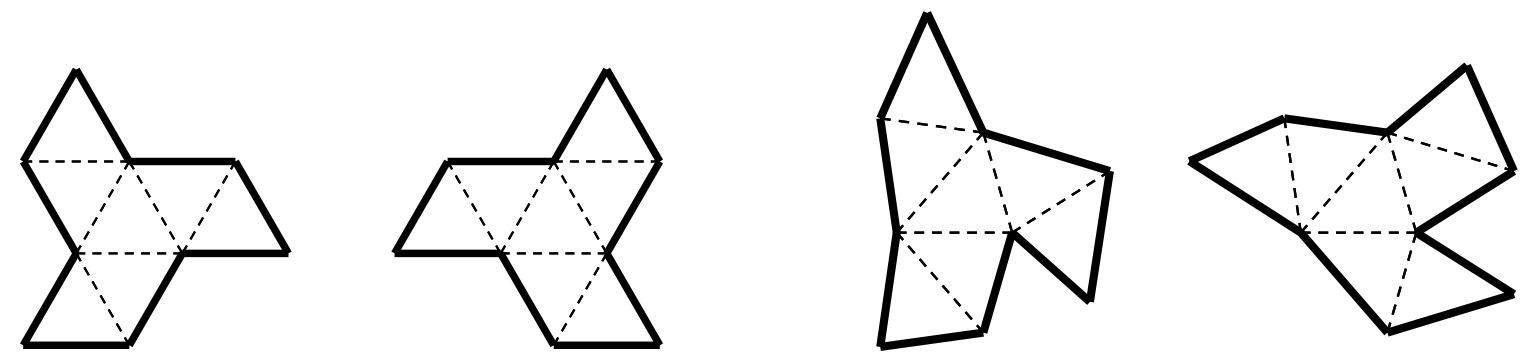

Figure 20 Pair $7_{1}$. Sunada triple $G=\operatorname{PSL}(3,2), G_{i}=\left\langle a_{i}, b_{i}, c_{i}\right\rangle, i=1,2$, with $a_{1}=\left(\begin{array}{ll}0 & 1\end{array}\right)(25)$,

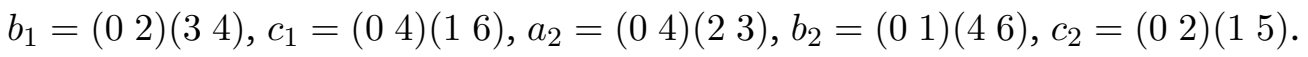
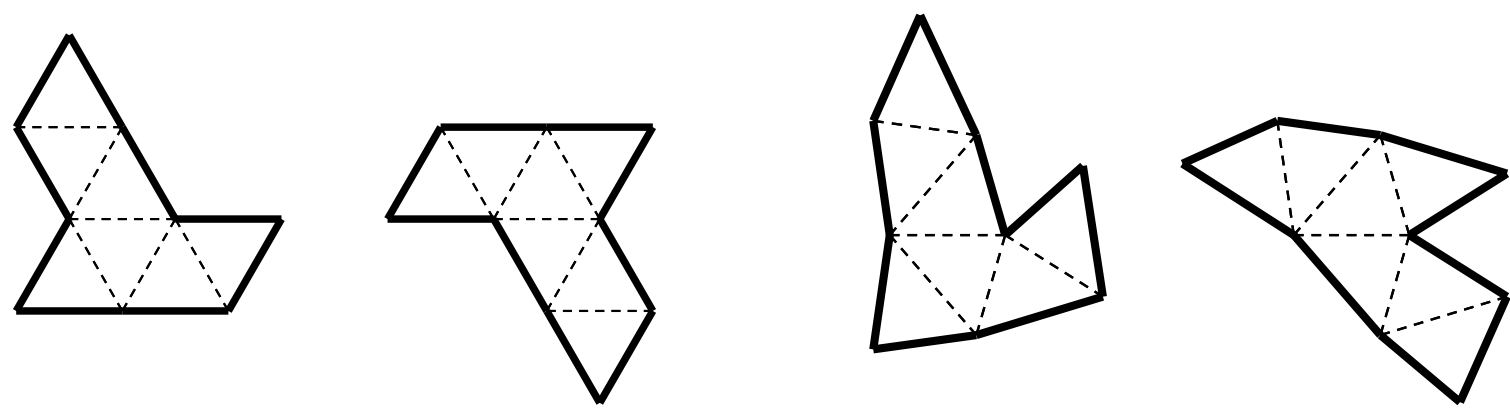

Figure 21 Pair $7_{2}$. Sunada triple $G=\operatorname{PSL}(3,2), G_{i}=\left\langle a_{i}, b_{i}, c_{i}\right\rangle, i=1,2$, with $a_{1}=\left(\begin{array}{ll}0 & 1\end{array}\right)(25)$,

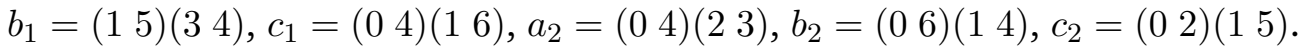

one less than $\pi / 2$. While it is possible to construct such a billiard, it is impossible to get a pair of planar billiards. Indeed, the role of the angles is exchanged from one billiard to the other, which leads to the condition that the three angles be less than $\pi / 3$.
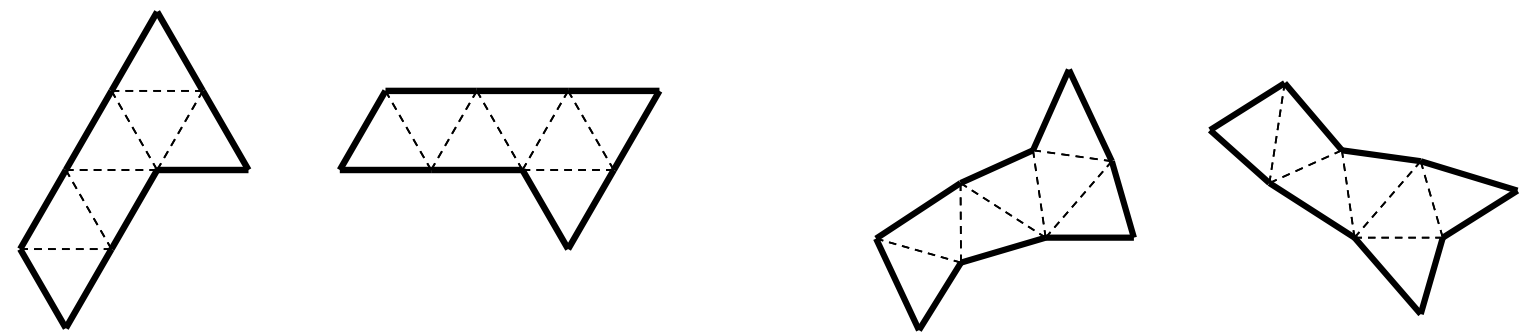

Figure 22 Pair $7_{3}$. Sunada triple $G=\operatorname{PSL}(3,2), G_{i}=\left\langle a_{i}, b_{i}, c_{i}\right\rangle, i=1,2$, with $a_{1}=(25)(46)$,

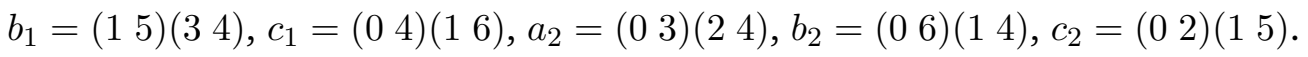



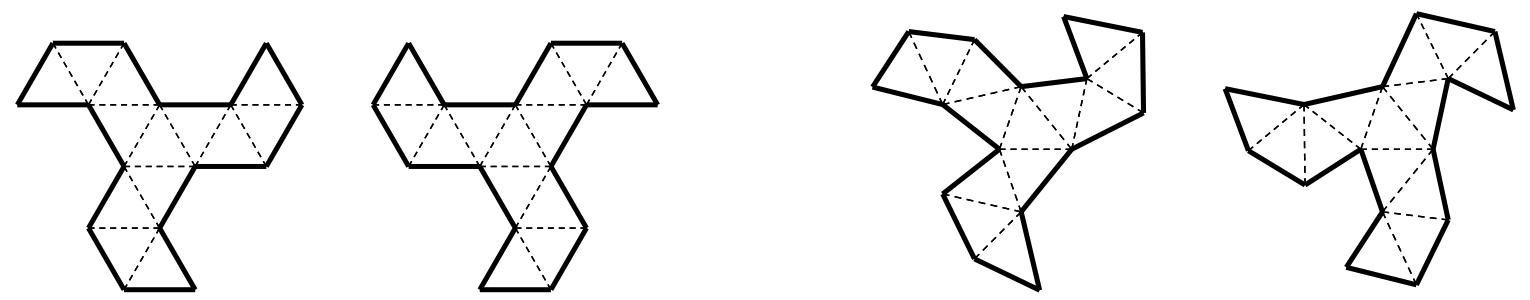

Figure 23 Pair $13_{1}$. Sunada triple $G=\operatorname{PSL}(3,3), G_{i}=\left\langle a_{i}, b_{i}, c_{i}\right\rangle, i=1,2$, with $a_{1}=$ $\left(0\right.$ 12)(1 10)(3 5)(6 7), $b_{1}=\left(\begin{array}{ll}0 & 10\end{array}\right)(2 \quad 9)(3 \quad 4)(5 \quad 8), c_{1}=\left(\begin{array}{lll}0 & 4\end{array}\right)\left(\begin{array}{lll}1 & 6\end{array}\right)\left(2\right.$ 11)(9 12), $a_{2}=$ $(04)\left(2\right.$ 3)(6 8)(9 10), $b_{2}=\left(\begin{array}{ll}0 & 12\end{array}\right)(14)\left(5\right.$ 11)(6 9), $c_{2}=\left(\begin{array}{lll}0 & 10\end{array}\right)(15)(2 \quad 7)(312)$.
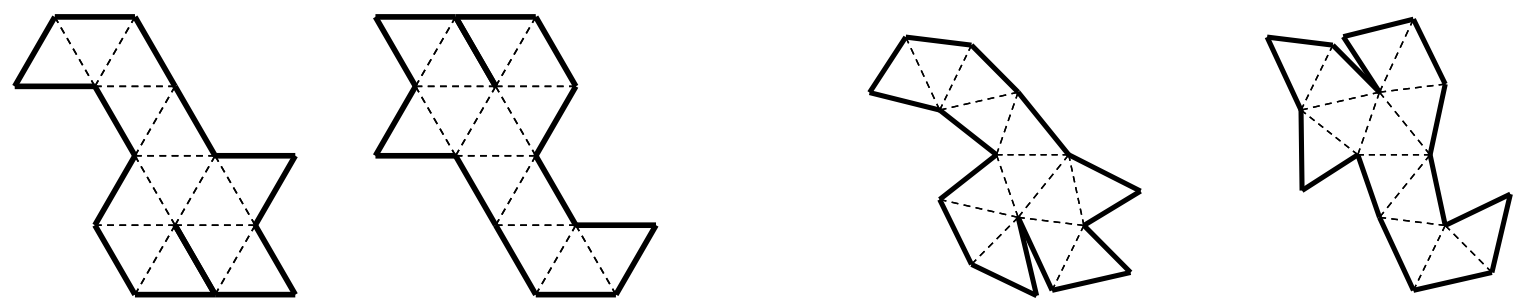

Figure 24 Pair $13_{2}$. Sunada triple $G=\operatorname{PSL}(3,3), G_{i}=\left\langle a_{i}, b_{i}, c_{i}\right\rangle, i=1,2$, with $a_{1}=$

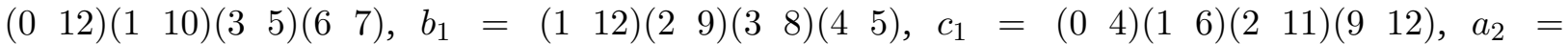

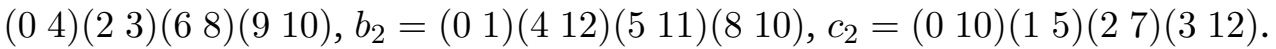
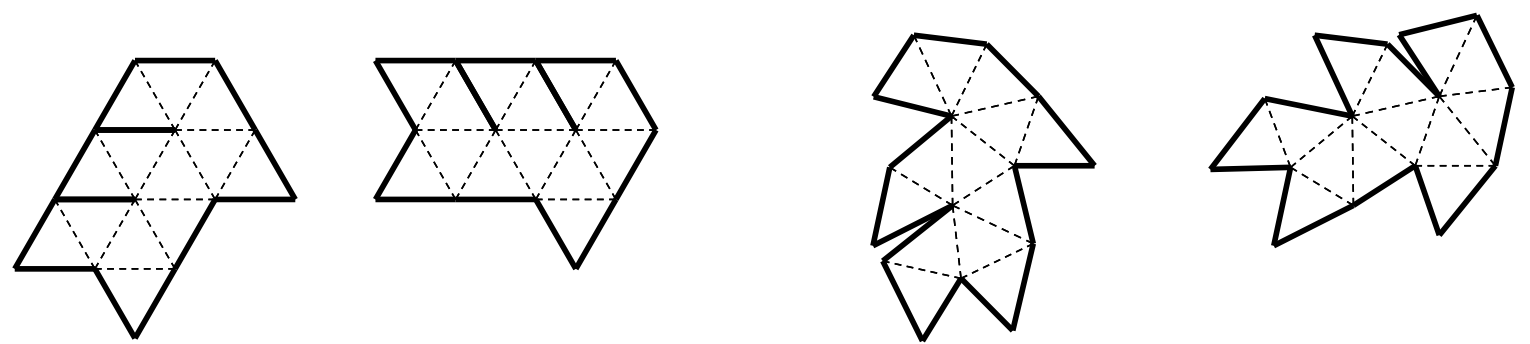

Figure 25 Pair $13_{3}$. Sunada triple $G=\operatorname{PSL}(3,3), G_{i}=\left\langle a_{i}, b_{i}, c_{i}\right\rangle, i=1,2$, with $a_{1}=$ $(1 \quad 7)(3 \quad 5)(4 \quad 9)(6 \quad 10), b_{1}=\left(\begin{array}{lll}1 & 12\end{array}\right)(2 \quad 9)(3 \quad 8)(4 \quad 5), c_{1}=\left(\begin{array}{lll}0 & 4\end{array}\right)\left(\begin{array}{lll}1 & 6\end{array}\right)\left(2\right.$ 11)(9 12), $a_{2}=$

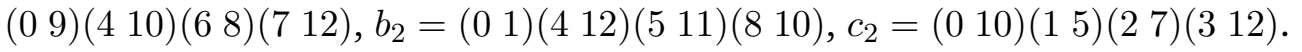
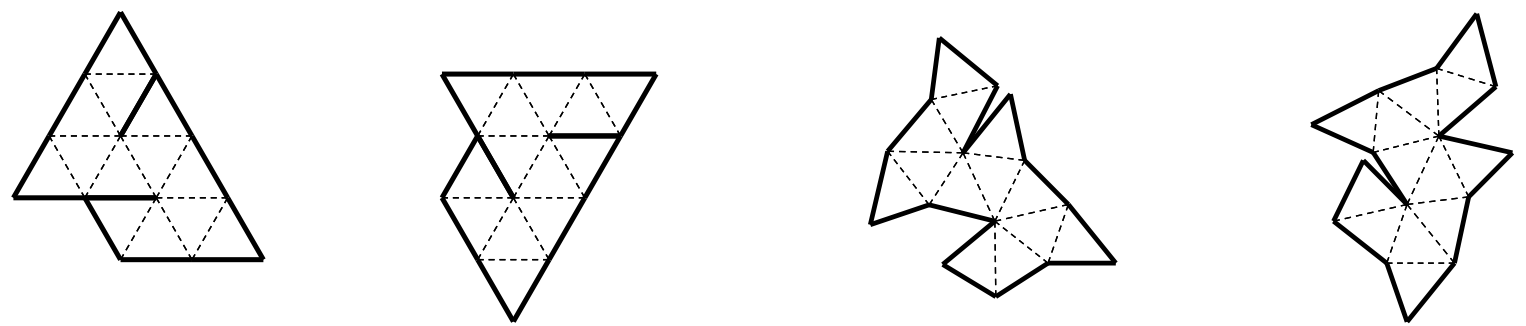

Figure 26 Pair 134. Sunada triple $G=\operatorname{PSL}(3,3), G_{i}=\left\langle a_{i}, b_{i}, c_{i}\right\rangle, i=1,2$, with $a_{1}=$

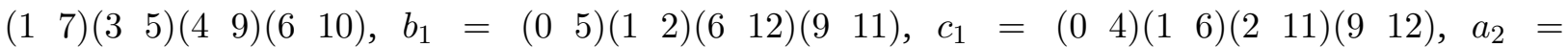

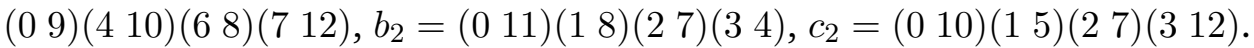



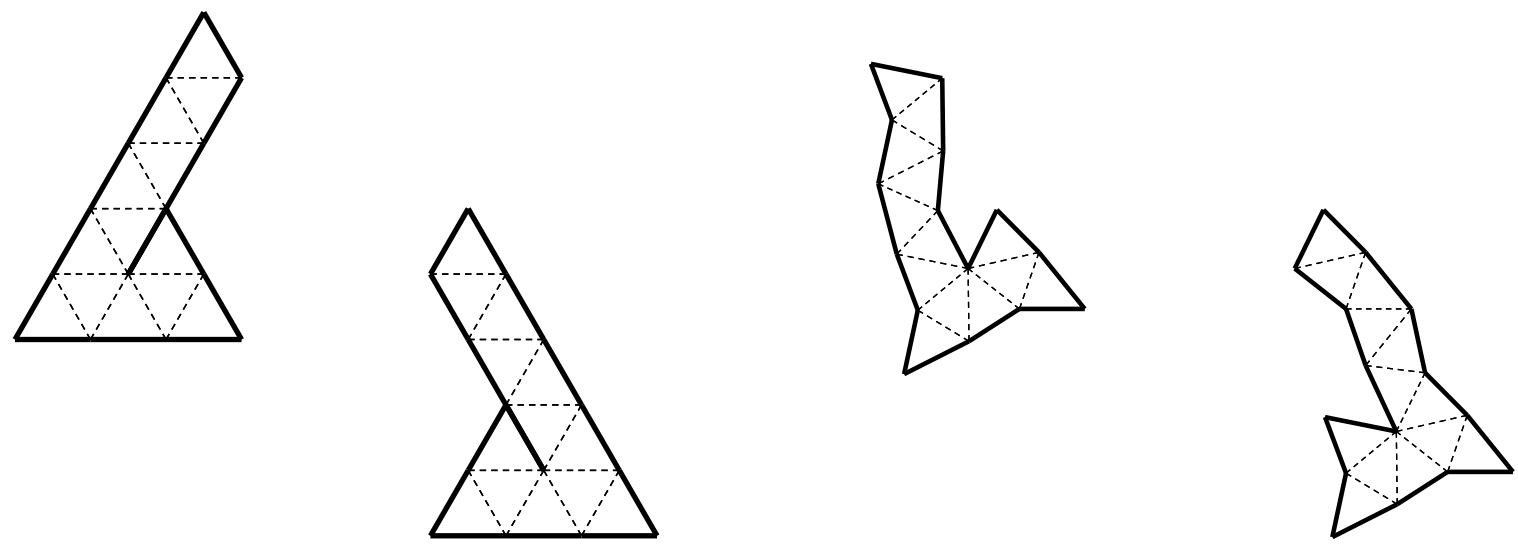

Figure 27 Pair $13_{5}$. Sunada triple $G=\operatorname{PSL}(3,3), G_{i}=\left\langle a_{i}, b_{i}, c_{i}\right\rangle, i=1,2$, with $a_{1}=$ $(1 \quad 7)(3 \quad 5)(4 \quad 9)(6 \quad 10), b_{1}=\left(\begin{array}{lll}0 & 5\end{array}\right)(1 \quad 2)(6 \quad 12)\left(9\right.$ 11),$c_{1}=\left(\begin{array}{lll}0 & 4\end{array}\right)\left(\begin{array}{lll}1 & 6\end{array}\right)\left(2\right.$ 11)(9 12), $a_{2}=$

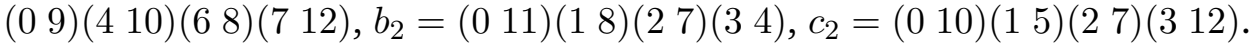



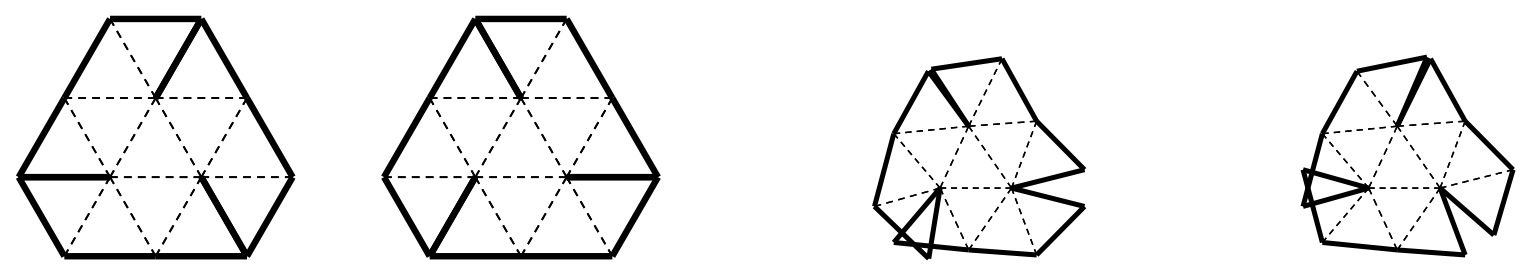

Figure 28 Pair $13_{6}$. Sunada triple $G=\mathbf{P S L}(3,3), G_{i}=\left\langle a_{i}, b_{i}, c_{i}\right\rangle, i=1,2$, with $a_{1}=$

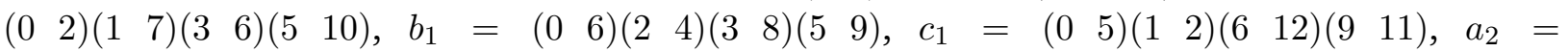

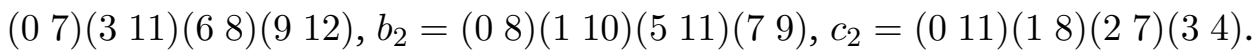
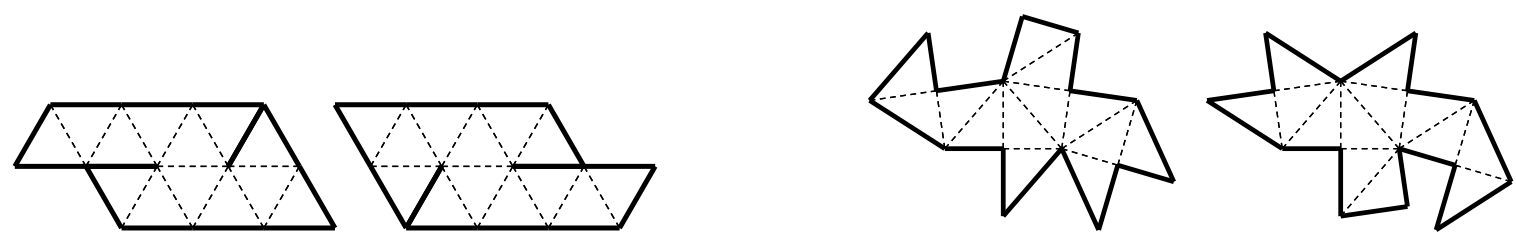

Figure 29 Pair 137. Sunada triple $G=\operatorname{PSL}(3,3), G_{i}=\left\langle a_{i}, b_{i}, c_{i}\right\rangle, i=1,2$, with $a_{1}=$

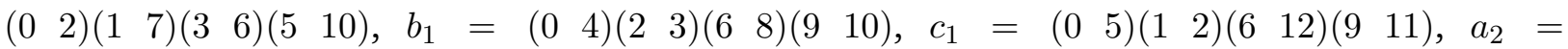

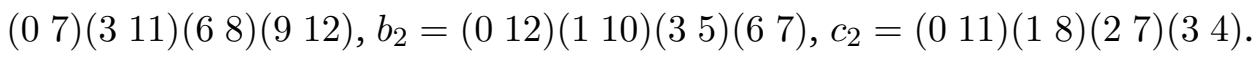
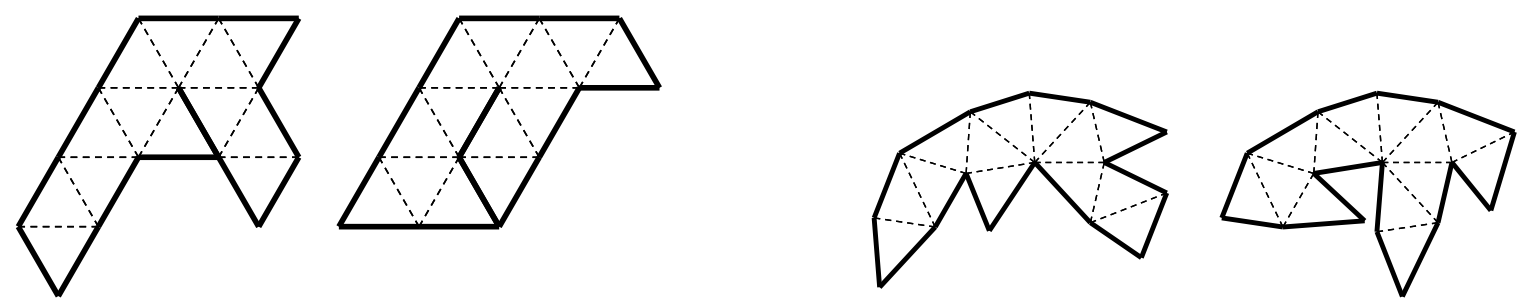

Figure 30 Pair 138 . Sunada triple $G=\operatorname{PSL}(3,3), G_{i}=\left\langle a_{i}, b_{i}, c_{i}\right\rangle, i=1,2$, with $a_{1}=$

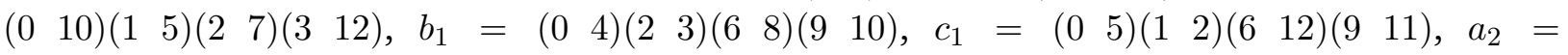

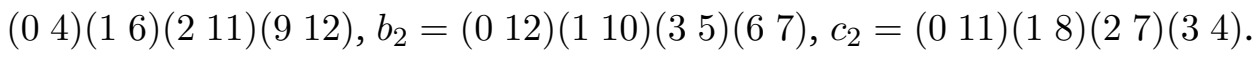
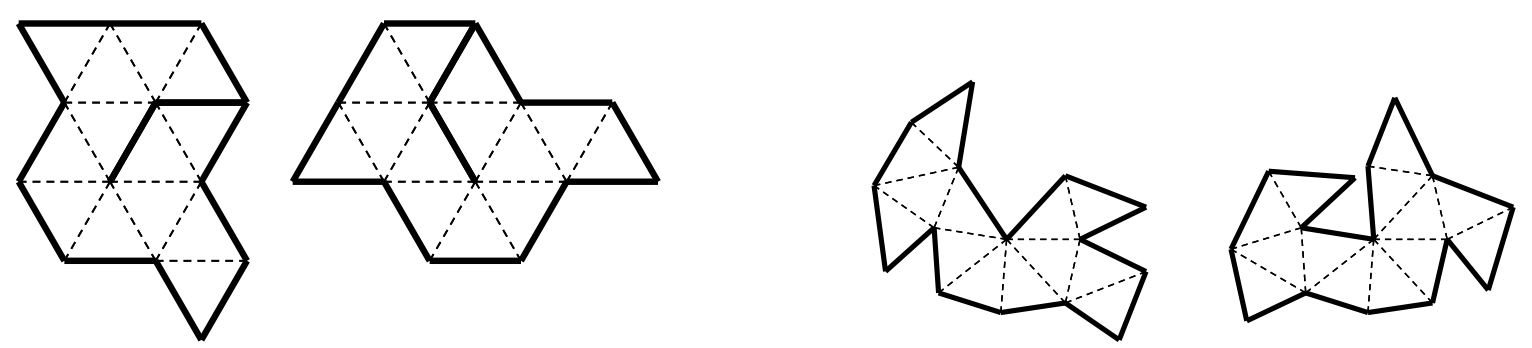

Figure 31 Pair 139 . Sunada triple $G=\operatorname{PSL}(3,3), G_{i}=\left\langle a_{i}, b_{i}, c_{i}\right\rangle, i=1,2$, with $a_{1}=$

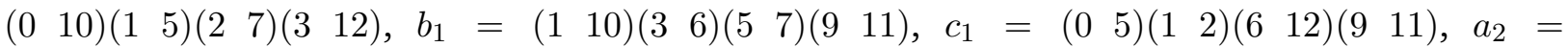

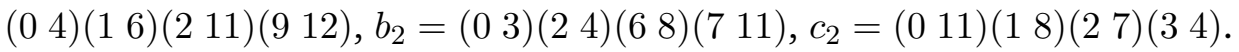



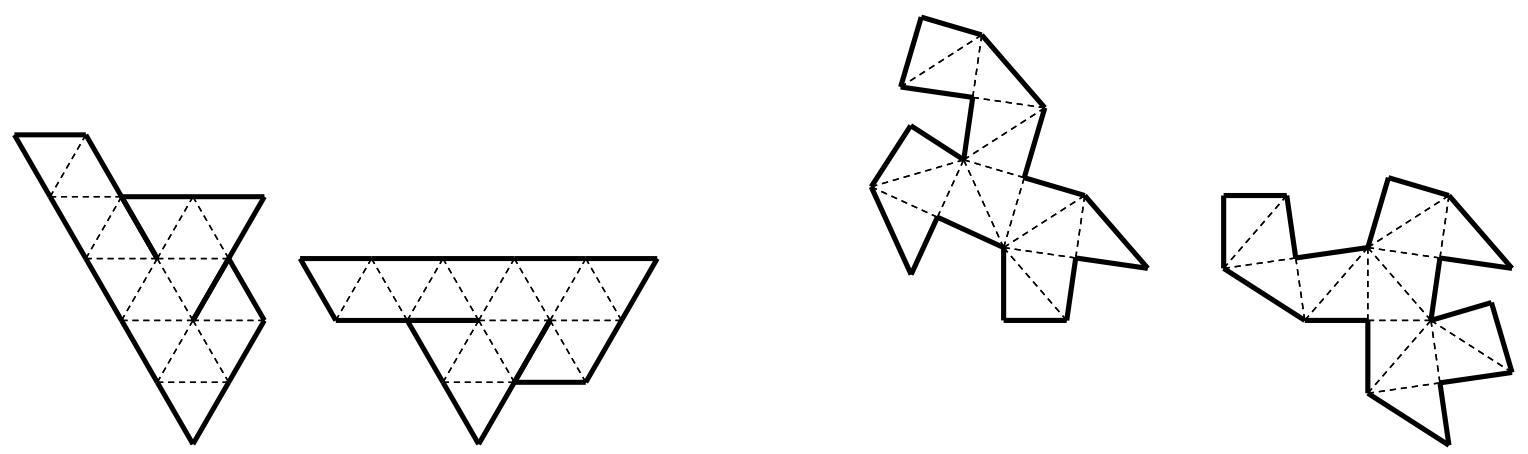

Figure 32 Pair $15_{1}$. Sunada triple $G=\operatorname{PSL}(1,2), G_{i}=\left\langle a_{i}, b_{i}, c_{i}\right\rangle, i=1,2$, with $a_{1}=$

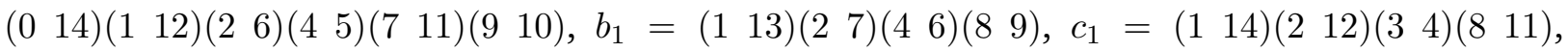

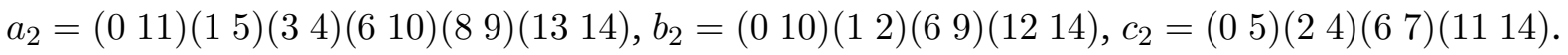
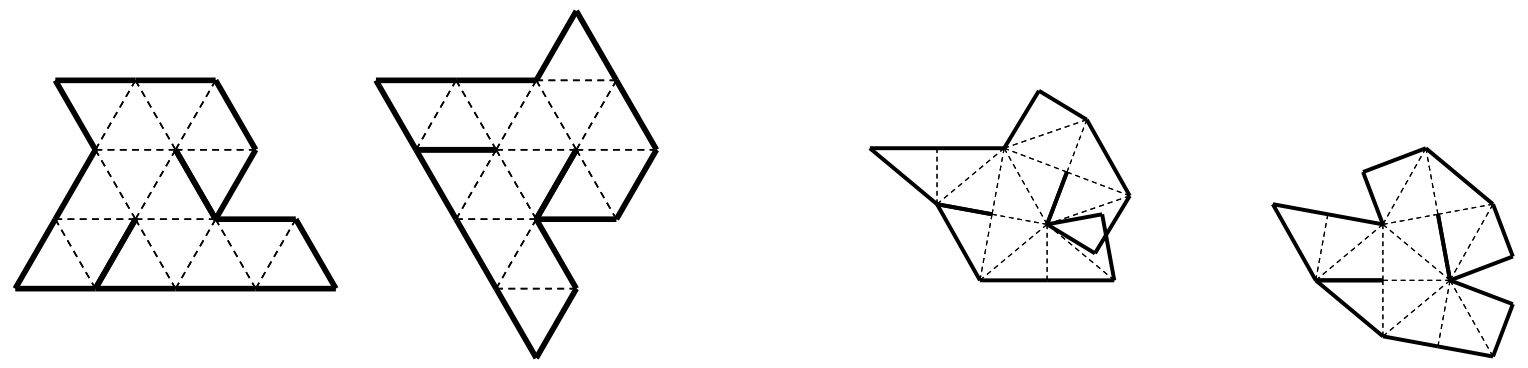

Figure 33 Pair $15_{2}$. Sunada triple $G=\operatorname{PSL}(1,2), G_{i}=\left\langle a_{i}, b_{i}, c_{i}\right\rangle, i=1,2$, with $a_{1}=$

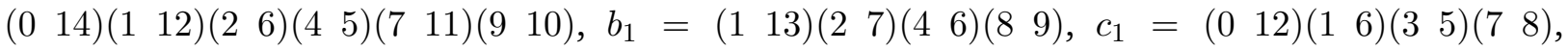

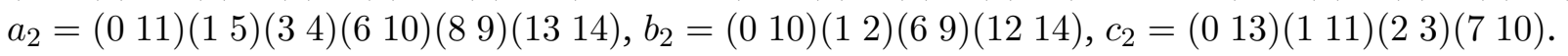
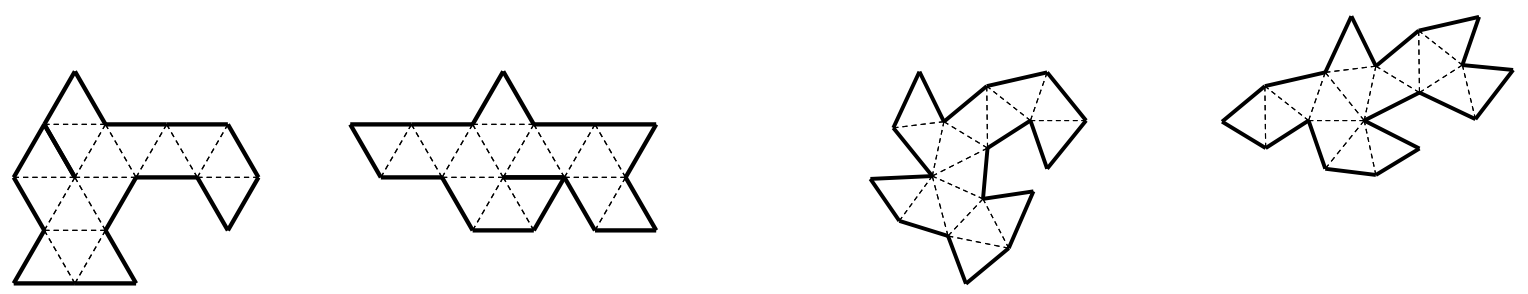

Figure 34 Pair $15_{3}$. Sunada triple $G=\operatorname{PSL}(1,2), G_{i}=\left\langle a_{i}, b_{i}, c_{i}\right\rangle, i=1,2$, with $a_{1}=$

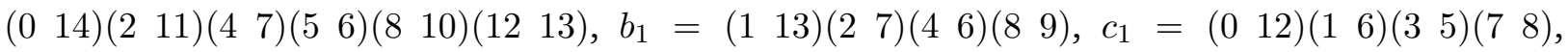

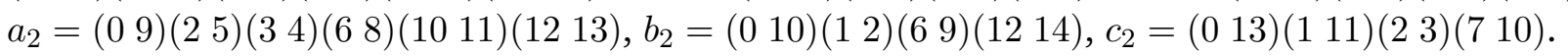



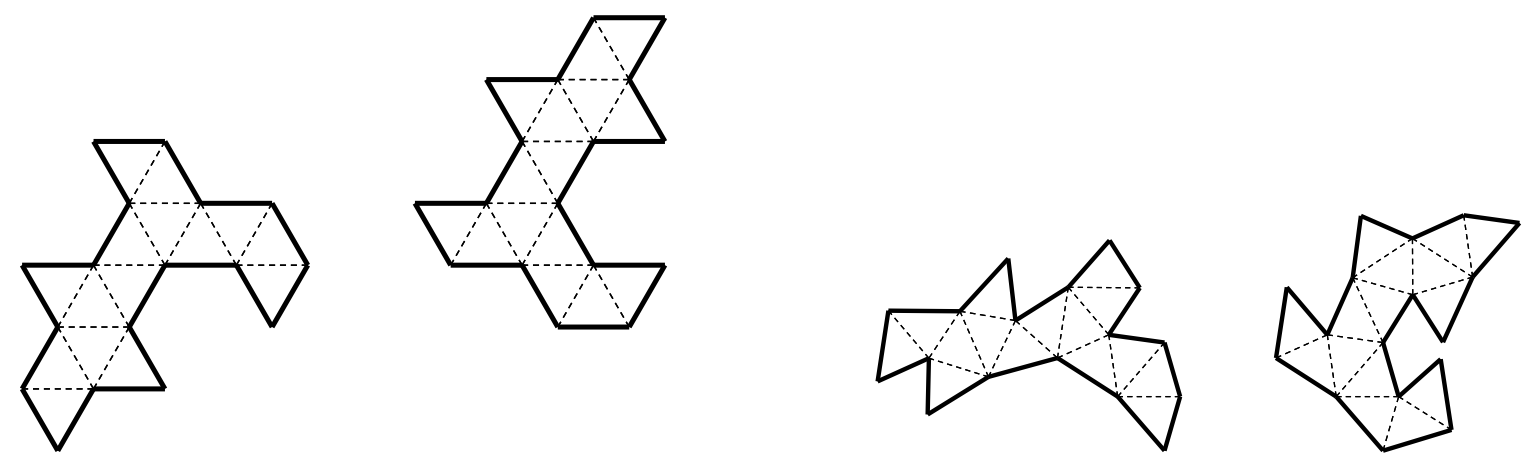

Figure 35 Pair $15_{4}$. Sunada triple $G=\operatorname{PSL}(1,2), G_{i}=\left\langle a_{i}, b_{i}, c_{i}\right\rangle, i=1,2$, with $a_{1}=$

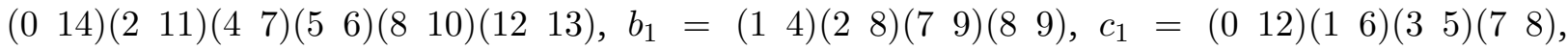

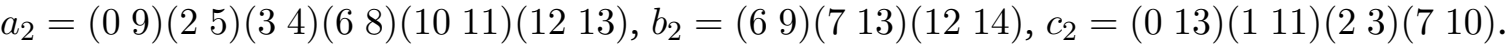
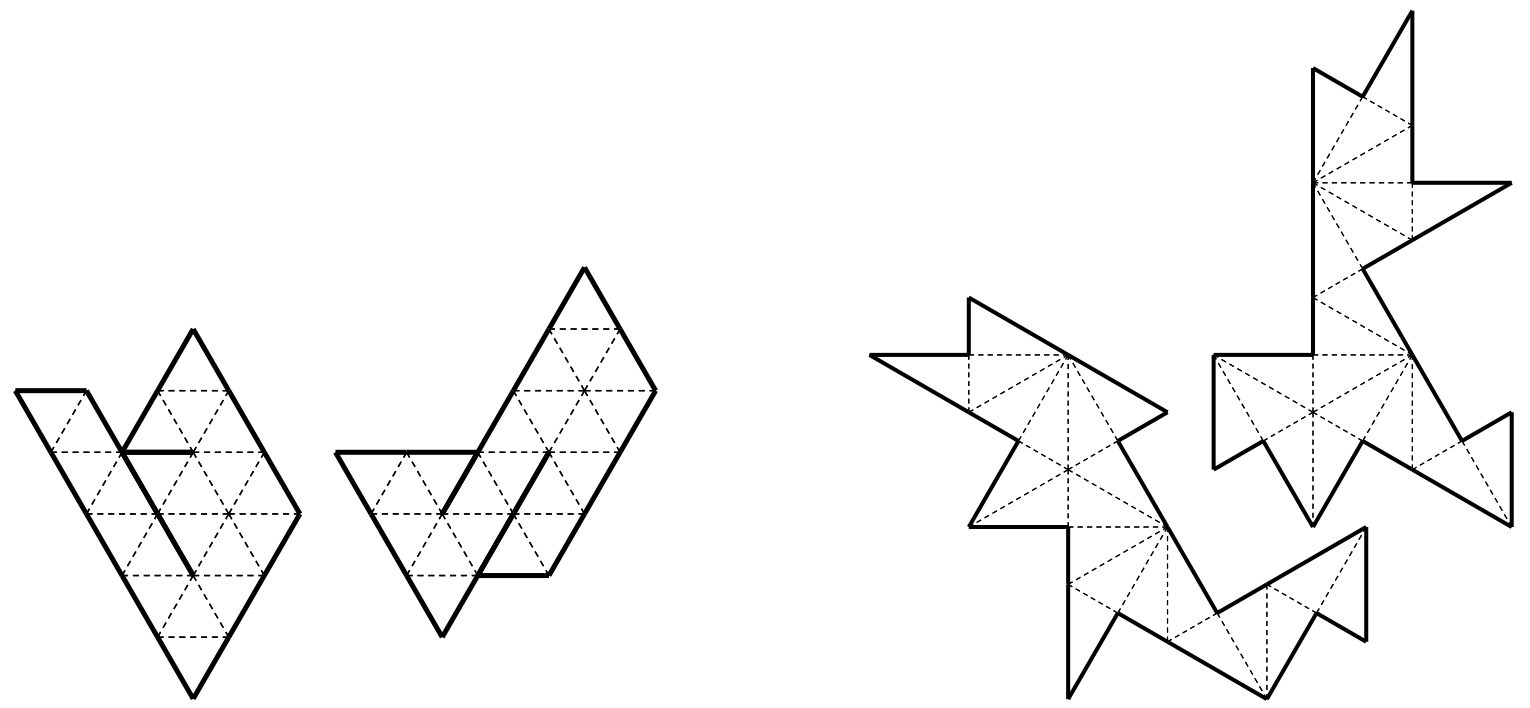

Figure 36 Pair $21_{1}$. Sunada triple $G=\operatorname{PSL}(3,4), G_{i}=\left\langle a_{i}, b_{i}, c_{i}\right\rangle, i=1,2$, with $a_{1}=$ (2 7)(3 11)(5 12)(8 18)(13 14)(15 17)(16 20), $b_{1}=\left(\begin{array}{lll}0 & 17\end{array}\right)(3 \quad 8)(4$ 12)(6 13)(9 19)(14 15)(16 18), $c_{1}=\left(\begin{array}{l}1 \\ 1\end{array}\right)\left(2\right.$ 16)(4 11)(5 19)(7 14)(10 17)(13 20), $a_{2}=\left(\begin{array}{l}0 \\ 0\end{array}\right)(4$ 17)(7 12)(9 16)(10 20)(11 13)(15 19), $b_{2}=\left(0\right.$ 20)(3 16)(6 11)(8 15)(9 19)(10 12)(14 18), $c_{2}=\left(\begin{array}{l}1 \\ \text { 8 }\end{array}\right)(2$ 16)(4 11)(5 19)(7 14)(10 17)(13 20). 


\section{References}

A. E. Brouwer, A. M. C., and A. Neumaier, 1989, Distance-Regular Graphs, volume 3.18 (Springer, Heidelberg).

Bailey, P. B., and F. H. Brownell, 1962, J. Math. Anal. Appl. 4, 212.

Balian, R., and C. Bloch, 1974, Ann. Phys. (N. Y. ) 85, 514.

Baltes, H., and E. R. Hilf, 1976, Spectra of Finite Systems (Bibliographisches Institut, Mannheim).

Bérard, P., 1989, Astérisque 177-178, 127.

Bérard, P., 1992, Math. Ann. 292, 547.

Bérard, P., 1993, J. London Math. Soc. 48, 565.

Berry, M. V., 1981, Eur. J. Phys. 2, 91.

Berry, M. V., 1985, Proc. Roy. Soc. A 400, 229.

Berry, M. V., 1991, in Chaos and Quantum Mechanics, edited by M.-J. Giannoni, A. Voros, and J. Zinn-Justin (North Holland, Amsterdam), les Houches Summer School Lectures, Session LII, 1989.

Berry, M. V., and P. J. Richens, 1981, Physica D 2, 495.

Berry, M. V., and M. Tabor, 1976, Proc. Roy. Soc. Lond. 349, 101.

Berry, M. V., and M. Tabor, 1977, Proc. Roy. Soc. Lond. 356, 375.

Betcke, T., and L. N. Trefethen, 2005, SIAM Review 47(3), 469.

Bohigas, O., M.-J. Giannoni, and C. Schmit, 1984, Phys. Rev. Lett. 52, 1.

Brownell, F. H., 1957, J. Math. Mech. 6, 119.

Buser, P., J. Conway, and P. Doyle, 1994, International Mathematics Research Notices 9, 391.

C. Gordon, D. W., and S. Wolpert, 1992, Invent. Math. 110, 1.

Chapman, S. J., 1995, Am. Math. Monthly 102(2), 124.

Courant, R., and D. Hilbert, 1953, Methods of Mathematical Physics, volume I (Interscience, New York).

D. M. Cvetkovic, M. D., and H. Sachs, 1998, Spectra of Graphs: Theory and Applications (Wiley, New York), 3rd rev. enl. ed.

van Dam, E. R., and W. H. Haemers, 2003, Linear Algebra Appl. 373, 241.

Descloux, J., and M. Tolley, 1983, Comput. Methods Appl. Mech. Engrg. 39, 37.

Driscoll, T. A., 1997, SIAM Rev. 39(1), 1. 
Driscoll, T. A., and H. P. W. Gottlieb, 2003, Phys. Rev. E 68, 016702.

Even, C., and P. Pieranski, 1999, Europhys. Lett. 47, 531.

Feit, W., and D. Higman, 1964, J. Algebra 1, 114.

Fox, L., P. Henrici, and C. Moler, 1967, SIAM J. Numer. Anal. 4, 89.

Fulling, S. A., and P. Kuchment, 2005, Inverse Problems 21, 1391.

Garabedian, P. R., 1998, Partial Differential Equations (AMS Chelsea Publ., Amer. Math. Soc., Providence, RI).

Garabedian, P. R., and M. Schiffer, 1952, J. d'Anal. Math. 2, 281.

Gassmann, F., 1926, Math. Z. 25, 665.

Georgeot, B., and R. E. Prange, 1995, Phys. Rev. Lett. 74(15), 2851.

Gerst, I., 1970, Acta Arith. 27, 121.

Giraud, O., 2004, J. Phys. A: Math. Gen. 37(7), 2751.

Giraud, O., 2005a, J. Phys. A 38, L477.

Giraud, O., 2005b, J. Phys. A: Math. Gen. 38, L477.

Gnutzmann, S., U. Smilansky, and N. Sondergaard, 2005, J. Phys. A: Math. Gen. 38, 8921.

Gordon, C., D. Webb, and S. Wolpert, 1992, Invent. math. 110, 1.

Gordon, C. S., 1986, Contemp. Math. 51, 63.

Gorenstein, D., 1980, Finite Groups. Second edition (Chelsea Publishing Co., New York).

Gottlieb, H. P. W., and J. P. McManus, 1998, Journal of Sound and Vibration 212(2), 253.

Guhr, T., A. Müller-Groeling, and H. Weidenmüller, 1998, Phys. Rep. 299, 189.

Gutkin, E., and C. Judge, 2000, Duke Math. J. 103(2), 191.

Gutzwiller, M., 1990, Chaos in classical and quantum mechanics (Springer Verlag, New York).

Gutzwiller, M. C., 1991, in Chaos and Quantum Mechanics, edited by M.-J. Giannoni, A. Voros, and J. Zinn-Justin (North Holland, Amsterdam), les Houches Summer School Lectures, Session LII, 1989.

Hannay, J. H., and A. Thain, 2003, J. Phys. A: Math. Gen. 36, 4063.

Hirschfeld, J. W. P., 1998, Projective Geometries over Finite Fields. Second edition (The Clarendon Press, Oxford University Press, New York).

Hughes, D. R., and F. C. Piper, 1973, Projective Planes (Springer-Verlag, New York/Heidelberg/Berlin).

J. A. Thas, K. T., and H. V. Maldeghem, 2006, Translation Generalized Quadrangles, volume 26 
(World Scientific, Singapore).

Jakobson, D., M. Levitin, N. Nadirashvili, and I. Polterovich, 2006, J. Comp. Appl. Math. 194(1), 141.

Kač, M., 1966, Am. Math. Monthly 73(4), 1.

Keller, J., 1962, J. Opt. Soc. Am. 52, 116.

Keller, J. B., and S. I. Rubinow, 1960, Ann. Phys. (N.Y.) 9, 24.

Komatsu, K., 1976, Kodai Math. Sem. Rep. 28, 78.

L. Ivanov, L. K., and S. Krein, 1977, Boundary Value Problems in Variable Domains, volume 19 (Math. Inst. of Lithuanian Acad. Sci., Vilnius).

Levitin, M., L. Parnovski, and I. Polterovich, 2006, J. Phys. A: Math. Gen. 39, 2073.

Maldeghem, H. V., 1998, Generalized Polygons, volume 93 (Birkhäuser-Verlag, Basel/Boston/Berlin).

McKean, H. P., and I. M. Singer, 1967, J. Diff. Geom. .

Mehta, M. L., 1990, Random Matrix Theory (Springer, New York).

Melrose, R. B., 1996, Proc. Centre Math. Appl. Austral. Nat. Univ. 34, 137.

Milnor, J., 1964, Proc. Natl. Acad. Sc. U.S.A 52, 542.

Okada, Y., and A. Shudo, 2001a, J. Phys. A: Math. Gen. 34, 5911.

Okada, Y., and A. Shudo, 2001b, J. Phys. A: Math. Gen. 34, 5911.

Okada, Y., A. Shudo, S. Tasaki, and T. Harayama, 2005a, J. Phys. A: Math. Gen. 38, L163.

Okada, Y., A. Shudo, S. Tasaki, and T. Harayama, 2005b, J. Phys. A: Math. Gen. 38, 6675.

Payne, S. E., and J. A. Thas, 1984, Finite Generalized Quadrangles, volume 110 (Pitman Advanced Publishing Program, Boston/London/Melbourne).

Pleijel, A., 1953-1954, Arkiv Matematik 2, 553.

Pleijel, A., 1956, Commun. Pure Appl. Math. 9, 543.

Porter, C. E., 1965, Statistical Theories of Spectra: Fluctuations (Academic Press, New York).

Rauch, J., 1978, Amer. Math. Monthly 85, 359.

Segre., B., 1961, Lectures on Modern Geometry. With an appendix by Lucio Lombardo-Radice, volume 7 (Edizioni Cremonese, Rome).

Sleeman, B. D., 1982, IMA Journal of Applied Mathematics 29, 113.

Sleeman, B. D., and C. Hua, 2000, Rev. Mat. Iberoamericana 16, 351.

Smithies, F., 1962, Integral Equations, volume 49 (Cambridge University Press, Cambridge). 
Solomon, R., 2001, Bull. Amer. Math. Soc. 38, 315.

Sridhar, S., 1991, Phys. Rev. Lett. 67, 785.

Sridhar, S., and E. J. Heller, 1992, Phys. Rev. A 46, R1728.

Sridhar, S., D. O. Hogenboom, and B. A. Willemsen, 1992, J. Stat. Phys. 68, 239.

Sridhar, S., and A. Kudrolli, 1994, Phys. Rev. Lett. 72(14), 2175.

Stewardson, K., and R. T. Waechter, 1971, Proceedings of the Cambridge Philosophical society 69, 353.

Sunada, T., 1985, Ann. Math. 121, 169.

Tasaki, S., T. Harayama, and A. Shudo, 1997, Phys. Rev. E 56(1), R13.

Thain, A., 2004, Eur. J. Phys. 25, 633.

Thas, K., ????a, Submitted .

Thas, K., ????b, Submitted .

Thas, K., ????c, Submitted .

Thas, K., ????d, Submitted .

Thas, K., 2004, Symmetry in Finite Generalized Quadrangles, volume 1 (Birkhäuser).

Thas, K., 2006a, J. Phys. A: Math. Gen. 39, L385.

Thas, K., 2006b, J. Phys. A: Math. Gen. 39, 13237.

Thas, K., 2007, J. Phys. A: Math. Theor. 40, 7233.

Thas, K., and H. V. Maldeghem, ????, Trans. Amer. Math. Soc. .

Tits, J., 1959, Inst. Hautes Etudes Sci. Publ. Math. 2, 13.

Tits, J., 1974, Buildings of Spherical Type and Finite BN-Pairs, volume 386 (Springer, Berlin).

Vattay, G., A. Wirzba, and P. Rosenqvist, 1994, Phys. Rev. Lett. 73(17), 2304.

Vorobets, Y. B., 1996, Russian Math. Surveys 51(5), 779.

Wintgen, D., 1987, Phys. Rev. Lett. 58, 1589.

Witt, E., 1941, Abh. Math. Sem. Univ. Hamburg 14, 323.

Wolpert, S., 1978, Trans. Amer. Math. Soc. 244, 313.

Wu, H., D. W. L. Sprung, and J. Martorell, 1995, Phys. Rev. E 51(1), 703.

Zaremba, S., 1927, Journal de mathématiques pures et appliquées 92(6), 127.

Zaremba, S., février 1909, Bulletin international de l'Académie des sciences de Cracovie , 125.

Zelditch, S., 2004, Surv. Differ. Geom. IX, 401.

Zemlyakov, A. B., and A. N. Katok, 1976, Math. Notes 18, 760. 